\title{
STEADY SOLUTIONS OF THE NAVIER-STOKES EQUATIONS REPRESENTING PLANE FLOW IN CHANNELS OF VARIOUS TYPES
}

\author{
BY \\ C. J. AMICK(1) and L. E. FRAENKEL( ${ }^{(2)}$ \\ Mathematics Division, University of Sussex, Brighton, England
}

\section{Table of Contents}

1. Introduction

1.1. Background . . . . . . . . . . . . . . . . . 84

1.2. The boundary-value problem . . . . . . . . . . 85

1.3. Channels of various types . . . . . . . . . . . . . . 87

1.4. Results and methods . . . . . . . . . . . . . . . 89

2. Preliminaries

2.1. Further notation and terminology . . . . . . . . . . 91

2.2. The spaces $H(\Omega)$ and $V(\Omega)$. . . . . . . . . . . . . . . . 93

2.3. Identities implied by the conformal map $F$. . . . . . . . . 95

2.4. Two properties of channels of type II . . . . . . . . . . . . 97

3. Existence of weak solutions

3.1. A priori bounds . . . . . . . . . . . . . . . . . . . . 98

3.2. Existence of weak solutions . . . . . . . . . . . . . 103

3.3. An a posteriori estimate for weak solutions in channels of type III 104

4. Regularity of the weak solution . . . . . . . . . . . . . 108

5. Pointwise decay at infinity for channels of type $I^{\prime}$

5.1. Channels of type $I^{\prime}$. . . . . . . . . . . . . . . . . . . 109

5.2. Estimates in a boundary neighbourhood. . . . . . . . . . . 111

5.3. Estimates of the vorticity . . . . . . . . . . . . . 114

5.4. Averages over ares of $p$ and $|p|$. . . . . . . . . . . . . . 117

5.5. Pointwise decay of the pressure and velocity . . . . . . . . 120

6. Pointwise decay at infinity for channels of type III

6.1. Introductory remarks. . . . . . . . . . . . . . . 125

6.2. Estimates from the vorticity equation. . . . . . . . . 127

6.3. Decay rates . . . . . . . . . . . . . . . . . 131

(1) Present address: St. John's College, Cambridge, England. Research supported by a postdoctoral Fellowship of the United States National Science Foundation.

(2) Research supported by the Science Research Council of the United Kingdom. 
Appendix A. On the conformal mapping of a strip onto a channel

A.1. Examples of maps $F$. . . . . . . . . . . . . . . 135

A.2. End maps. . . . . . . . . . . . . . . . . . . . . 138

A.3. On perturbations of end maps . . . . . . . . . . . . . 143

Appendix B. Some details omitted from proofs . . . . . . . . . . . 148

References . . . . . . . . . . . . . . . . 150

\section{Introduction}

\subsection{Background}

Recent work of Heywood [21] has drawn attention (a) to questions of uniqueness of Navier-Stokes solutions for certain unbounded domains $\Omega$ in $\mathbf{R}^{N}$ that can be regarded as models of channels, tubes, or conduits of some kind, and (b) to the importance of prescribing, not merely the fluid velocity $u$ on the boundary $\partial \Omega$, but also some quantity like the flux $M$ (that is, the total volumetric flow rate, defined for $N=2$ by (1.3b) below) or the overall pressure difference. However, the existence theory for such domains (which have boundaries that are not compact) seems somewhat sparse relative to that for bounded and for exterior domains. (By an exterior domain we mean a connected open set that is the complement of a bounded set.) If we set aside the explicit (or nearly explicit) solutions for special cases in which, by virtue of a particular boundary $\partial \Omega$, the non-linear terms vanish (as in the parallel Poiseuille velocity field appropriate to infinite cylinders), or a similarity situation allows reduction to ordinary differential equations (as in the Jeffery-Hamel solutions for source or sink flow in plane wedge-shaped domains), then, as far as we are aware and according to what is stated in [9], [10], [23] and [26], the only existence theorems for steady flow in domains of the class in question are as follows. We state restrictions in terms of the Reynolds number: for $N=2$ (in two space dimensions), $R=M / v$ is the ratio of flux to kinematic viscosity; for $N=3$, we introduce a length $l$ characteristic of some cross-section of the domain, and define $R=M / v l$.

(i) For the case of a smooth tube $\Omega \subset \mathbf{R}^{3}$ consisting of two semi-infinite circular cylinders joined by an arbitrary central bounded portion, one prescribes zero velocity on the boundary $\partial \Omega$ and the appropriate Poiseuille velocities at infinity upstream and downstream. P. Patterson proved existence of a solution to this problem for sufficiently small Reynolds numbers, and obtained rates at which the Poiseuille velocities are approached far upstream and far downstream. (We have not seen these unpublished results, and can refer only to their description in a review article by Finn, [9], p. 150; there, the critical Reynolds number is not characterized in any way.)

(ii) In [4] and [5] both the two- and three-dimensional versions of the foregoing 
problem are treated, and for $N=3$ the cylinders may have non-circular cross-sections. Existence, exponential approach to the Poiseuille velocities, and regularity properties of the solution are established for $R<R_{0}$, where the critical Reynolds number $R_{0}$ is 116.5 for $N=2$, is 127.9 for $N=3$ and cylinders of circular cross-section, and is somewhat larger for domains symmetrical about a streamwise axis. The number $1 / R_{0}$, which re-appears in certain results of the present paper, is the supremum over the unit sphere in a Hilbert space of a quadratic functional, and also marks a stability boundary of Poiseuille flow in an infinite strip $(N=2)$ or cylinder $(N=3)$; in this stability context, it was first computed for $N=2$ by W. Orr in 1907 .

(iii) Symmetrical channels $\Omega \subset \mathbf{R}^{2}$ with slowly curving walls are considered in [12] and [14], the product of local channel width and local wall curvature being bounded by a small parameter $\varepsilon>0$. Under certain restrictions on Reynolds number and the local divergence angle of the channel walls, solutions are constructed as explicit asymptotic series, to a finite number of terms, in powers of $\varepsilon$, plus a remainder that is proved to be smaller; these solutions exhibit 'flow separation' from the walls.

(iv) In [21], pp. 98-100, Heywood proves existence, for sufficiently small Reynolds numbers, of steady generalized solutions representing flow through certain apertures and ducts in $\mathbf{R}^{3}$ that widen strongly at infinity. $\left({ }^{1}\right)$

\subsection{The boundary-value problem}

By a channel we mean an unbounded domain $\Omega \subset \mathbf{R}^{2}$ that (a) is simply connected, (b) has a boundary $\partial \Omega$, of class $C^{\infty}$, consisting of two unbounded components $\Gamma_{-}$and $\Gamma_{+}$ (the channel walls) such that dist $\left(\Gamma_{-}, \Gamma_{+}\right)>0$. In the present paper we tackle the general problem of steady Navier-Stokes solutions for such domains. To specify the flux we let $\gamma$ denote any smooth simple arc, in $\bar{\Omega}$, directed from a point of $\Gamma_{-}$to a point of $\Gamma_{+}$, with unit normal $n$ such that a positive (anti-clockwise) rotation through $\pi / 2$ transforms $n$ into the (directed) unit tangent, and with $d l$ denoting an element of arc length. We seek a solution $(u, p)$ of the steady Navier-Stokes equations,

$$
\begin{gathered}
-\nu D^{2} u+(u \cdot D) u=-D p \text { in } \Omega, \\
\operatorname{div} u \equiv D \cdot u=0 \text { in } \Omega,
\end{gathered}
$$

where $\Omega$ is a channel, such that

$$
\left.u\right|_{\partial \Omega}=0, \quad \int_{\gamma} u \cdot n d l=M>0 .
$$

(1) For a fifth item, see the note References added in proof. 
Here $v$ denotes the kinematic viscosity, a given (strictly) positive constant; $D=\left(D_{1}, D_{2}\right)=(\partial / \partial x, \partial / \partial y)$ the gradient operator, and $D^{2}$ the Laplacian; $u=\left(u_{1}, u_{2}\right): \bar{\Omega} \rightarrow \mathbf{R}^{2}$ the fluid velocity; and $p: \bar{\Omega} \rightarrow \mathbf{R}$ the ratio of modified pressure to constant density, 'modified' pressure meaning that the scalar potential of a given irrotational force field may be included.

When the local channel width tends to infinity far upstream and far downstream, we add the condition

$$
|u(z)| \rightarrow 0 \text { as }|z| \rightarrow \infty \text { in } \Omega \text {. }
$$

Here $z$ denotes points $(x, y) \in \mathbf{R}^{2}$, which we identify with points $x+i y \in \mathbf{C}$ wherever this is appropriate. When the width tends to (positive) limits at a certain rate, we demand that

the appropriate Poiseuille velocities be approached, as in [4].

In what follows, '(1.4)' will mean either (1.4a) or (1.4b), according to the form of $\Omega$. The words 'local channel width', 'far upstream' and 'far downstream' will be given precise meaning presently; nevertheless, $(1.4 \mathrm{a})$ is to be interpreted loosely until we come to section 5. Definition 1.1 (a) will state that the 'local channel width tends to infinity' in an averaged sense, and in Definition 3.1 a flux-carrying velocity field will be required to satisfy (1.4a) only in the same averaged sense.

The restriction to the case $N=2$ is made for two reasons, as follows.

(i) Let $S$ denote the strip $\mathbf{R} \times(-1,1)$ and let $\Omega$ be a channel (Fig. 1). Then there exists a one-to-one conformal transformation, say

with

$$
\left.\begin{array}{rl}
z & =F^{\prime}(\zeta), \quad z=x+i y \quad \text { and } \quad \zeta=\xi+i \eta \\
\frac{d z}{d \zeta} & =F^{\prime}=h e^{i \theta} \quad\left(h=\left|F^{\prime}\right|\right),
\end{array}\right\}
$$

of $\bar{S}$ onto $\bar{\Omega}$ such that $\Gamma_{-}$and $\Gamma_{+}$are the images of $\mathbf{R} \times\{-1\}$ and $\mathbf{R} \times\{1\}$, respectively; the function $F$ is unique if $\xi_{0}=\operatorname{Re} F^{-1}\left(z_{0}\right)$ is specified for one point $z_{0} \in \Omega$. (Here $\operatorname{Re}$ and Im denote the real and imaginary parts respectively. The existence of $F$ follows from the Riemann mapping theorem and two elementary conformal transformations that map the unit disk, with $e^{i \alpha}$ and $e^{i \beta}$ representing the infinitely distant ends of $\Omega$, onto $S$ in the manner stated. To prove uniqueness, we consider two such maps $F_{1}$ and $F_{2}$, and apply the Phragmén-Lindelöf principle as in [25], pp. 97-99, to show that the function $\operatorname{Im} F_{2}^{-1} \circ F_{1}$ : $\bar{S} \rightarrow[-1,1]$ can only have the values $\eta$. A more elementary uniqueness proof, based on the fact that a conformal one-to-one map of the unit disk onto itself is a Möbius transformation, is also possible.) The map $F$ allows definition of types of channel, and construction of 


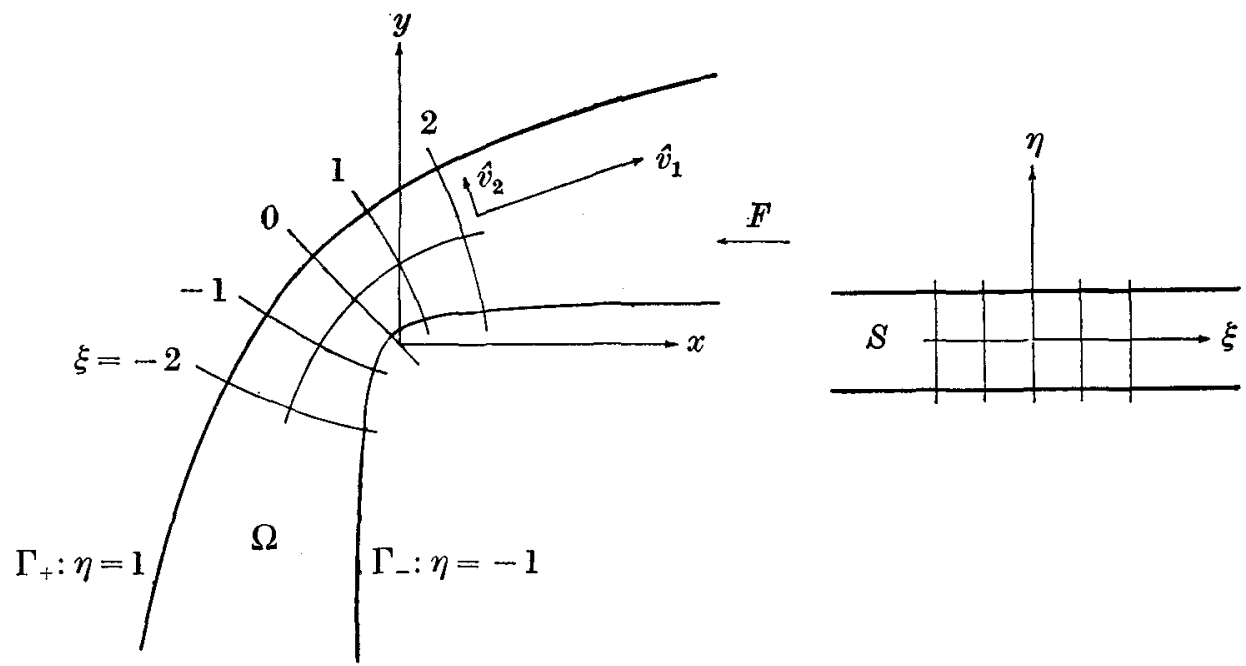

Fig. 1. Notation for the conformal map $F$. The velocity components $\hat{v}_{1}$ and $\hat{v}_{2}$, in the directions $h D \xi$ and $h D \eta$, are introduced in section 2.3. The particular channel $\Omega$ in the figure is of type III, but is on the borderline with type I (section 1.3 and Appendix A, (i)).

certain flux-carrying velocity fields essential to our proofs, with generality and ease, in a manner that has no obvious analogue in higher dimensions. We call the length $\int_{-1}^{1} h(\xi, \eta) d \eta$ of an $\operatorname{arc} \xi(x, y)=$ const. in $\Omega$ the local channel width; by limiting values far upstream (or far downstream) we mean limits as $\xi \rightarrow-\infty$ (or $\xi \rightarrow \infty)$.

(ii) For exterior domains $\Omega_{e}$ the analogue of (1.4), that $u$ is to tend to a prescribed constant velocity at infinity, demands treatments that are quite different for $N=2$ and for $N=3$. In the two-dimensional case, the question of whether a generalized solution satisfies this condition remains open, [10], [17], [18], except for sufficiently large viscosity $v,[11]$; in the three-dimensional case, the question has been settled completely, [6]. (One basic difference is in the space $H(\Omega)$ of divergence-free vector fields $v$ that vanish on $\partial \Omega$, with the Dirichlet norm: $\|v\|^{2}=\int_{\Omega}|D v|^{2}$. For $N=2$, there exist fields $v$ in $H\left(\Omega_{e}\right)$ such that $|v(z)| \rightarrow \infty$ as $|z| \rightarrow \infty$; for $N=3, H\left(\Omega_{e}\right)$ is embedded in $L_{6}\left(\Omega_{e} \rightarrow \mathbf{R}^{3}\right)$.) This feature of the exterior problem has an easier, but still serious, counterpart for channels and tubes whose width tends to infinity far upstream or downstream, and proofs that (1.4) holds pointwise form a substantial part of the present paper.

\subsection{Channels of various types}

It will be convenient to distinguish the gradient operator $D$ in $\Omega$ from that in $S$; we write $\nabla=\left(\nabla_{1}, \nabla_{2}\right)=(\partial / \partial \xi, \partial / \partial \eta)$ for the latter. 
Definition 1.1. Let $h: \bar{S} \rightarrow(0, \infty)$ be the arc-length function introduced in (1.5). We shall say that a channel $\Omega$ is

(a) of type $I$ if $1 / h$ and $|\nabla(1 / h)|$ belong to $L_{2}(S)$;

(b) of type $I I$ if $|\nabla \log h(\xi, \eta)| \rightarrow 0$ pointwise as $|\xi| \rightarrow \infty$, uniformly with respect to $\eta$, and $|\nabla(1 / h)| \in L_{2}(S)$;

(c) of type III if it is of type II and also $|\nabla \log h| \in L_{2}(S),|\nabla h| \in L_{q}(S)$ for some $q>2$, and $h(\xi, \eta) \leqslant$ const. $|\xi|^{\frac{1}{2}}$ for $|\xi|>1$.

We hasten to give intuitive meaning to this definition, and to indicate under what circumstances a given channel belongs to one of these types; further details are presented in Appendix A. Note first that $h \in C^{\infty}(\bar{S})$ and that, on any compact subset of $\bar{S}, h$ is bounded away from zero. (This may be proved by combining a classical theorem on conformal mapping, [7], pp. 96-97, with regularity theory for the solution of the Dirichlet problem $D^{2} \eta=0$ in $\Omega,\left.\eta\right|_{\Gamma_{-}}=-1$ and $\left.\eta\right|_{\Gamma_{+}}=1$. Observe that $1 / \hbar=|D \eta|$ if we write $h=\tilde{h} \circ F$.) Therefore the definition restriets only the behaviour of $h(\xi, \eta)$ as $|\xi| \rightarrow \infty$. Now consider symmetrical channels

$$
\Omega_{f}=\left\{(x, y) \in \mathbf{R}^{2} \mid x \in \mathbf{R},-f(x)<y<f(x)\right\}
$$

where

$$
f(x)= \begin{cases}\text { const. }(-x)^{k_{1}} & \text { for } x<-l_{1}-1 \\ \text { const. } x^{k_{2}} & \text { for } x>l_{2}+1\end{cases}
$$

$k_{j}$ and $l_{j}(j=1,2)$ being non-negative constants. Examples and a theorem in Appendix A show that $\Omega_{f}$ is of type I if $k_{j}>\frac{1}{3}$, of type II if $k_{j}<1$, and of type III if $k_{j} \leqslant \frac{1}{3}(j=1,2$ throughout). Thus the sets $I$ and II (of channels of types I and II respectively) intersect; but $I$ and III are disjoint because $1 / h \notin L_{2}(S)$ when $h(\xi, \eta) \leqslant$ const. $|\xi|^{\frac{1}{2}}$ for $|\xi|>1$. We have already defined local channel width as twice the mean value $\vec{h}(\xi)=\frac{1}{2} \int_{-1}^{1} h(\xi, \eta) d \eta$; if for the moment we ignore restrictions on $\nabla h$, we may loosely describe channels of type $I$ as those which widen strongly at infinity $\left(k_{j}>\frac{1}{3}\right)$ and channels of type III as those which widen feebly there $\left(k_{j} \leqslant \frac{1}{3}\right)$.

Regarding $\nabla h$. Recalling from (1.5) that $\log h+i \theta$ is a holomorphic function on $S$, we define

$$
\varkappa=h_{\xi} / h=\theta_{\eta}, \quad \lambda=h_{\eta} / h=-\theta_{\xi},
$$

where $(.)_{\xi}=\partial(.) / \partial \xi$. The mean value $\bar{x}(\xi)=\frac{1}{2} \int_{-1}^{1} \varkappa(\xi, \eta) d \eta$ then represents half the local divergence angle, $\theta(\xi, 1)-\theta(\xi,-1)$, of the channel walls, and $-\nabla(1 / h)=\left(\theta_{\eta},-\theta_{\xi}\right) / h$ re- 
presents the curvatures of the arcs $\xi=$ const. and $\eta=$ const. in $\Omega$.Accordingly, channels of type II have local divergence angles that tend to zero at infinity, and (like those of type I) have conformal coordinate lines $\xi=$ const. and $\eta=$ const. in $\Omega$ whose curvatures are in $L_{2}(S)$. For type III these conditions are strengthened. Note that, if $h$ and the curvatures are regarded as functions on $\Omega$, say $h \circ F^{-1}=\tilde{h}$ and $-\nabla(1 / h) \circ F^{-1}=(K, L)$, then it is merely $K / \hbar$ and $L / \hbar$ that are required to be in $L_{2}(\Omega)$, because $d \xi d \eta=d x d y / \hbar^{2}$; and $\hbar(z) \rightarrow \infty$ for most cases.

\subsection{Results and methods}

(i) For channels $\Omega$ of type $I$, a weak solution is obtained in section 3 for every Reynolds number by a variant of an artifice that originates in early work of Leray and is now standard for compact boundaries (see, for example, [9], pp. 128-129). Smoothness of weak solutions, for all types of channel, on compact subsets of $\bar{\Omega}$ is established by standard methods in section 4. There remains the question of the pointwise decay of $u$ at infinity in $\Omega$; adopting slightly stronger hypotheses about $h$ (the channel is then of type $\left.I^{\prime}\right)$, we prove in section 5 that (1.4a) holds, and that the pressure $p$ tends uniformly to finite limits far upstream and far downstream. This is done by use and extension of the considerable machinery built by Gilbarg and Weinberger [17], [18] for the twodimensional exterior problem; an essential tool in [17] is a one-sided maximum principle for the function $p+\frac{1}{2}|u|^{2}$. Thus, for channels of type $I^{\prime}$ and for every Reynolds number $R=M / v$, a classical (pointwise) solution of (1.1) to (1.4a) is established. However, our attempts to obtain a rate of decay for the velocity in this case have been wholly unsuccessful.

(ii) For channels of types $I I$ and III, solutions are obtained only for $R<R_{0}$, where $R_{0}$ is the critical Reynolds number described in (ii) of section 1.1. For type $I I I$ there emerges a rather precise description of the velocity and pressure at large distances. The dominant part of the velocity far upstream and downstream is a slightly distorted Poiseuille velocity $q$ of magnitude $\frac{3}{4} M\left(1-\eta^{2}\right) / h(\xi, \eta)$ and direction $D \xi(z)$ in $\Omega$, and the departures from this velocity, due to geometrical effects represented by $\nabla h$, can also be estimated. Note that the factor $1 / h(\xi, \eta)$ implies a rate of decay when the local channel width tends to infinity. It is also shown that $p(z) \rightarrow \pm \infty$ as $\xi \rightarrow \mp \infty$, respectively, and the pressure gradient is estimated.

Our method is as follows. In section 3 a weak solution $u=g+v$ is obtained for channels of type II by a variant of the method in [4]; the velocity field $g$ is constructed a priori to satisfy (1.2) to (1.4). On a certain compact subset of $\bar{\Omega}, g$ has the form used for type $\mathrm{I}$; at sufficiently large distances, $g$ is the distorted Poiseuille velocity $q$ described 
above. The Gilbarg-Weinberger approach to analysis of the far field is no longer applicable, because the pressure $p$ need not tend to limits far upstream and far downstream for channels of type II. On the other hand, the type of decay theory used in [5], which exploits the Green function of the linearization of the Navier-Stokes equations about the Poiseuille solution, requires, for control of the non-linear terms, not merely a bound for the Dirichlet integral $\int_{\Omega}|D v|^{2}$ of the second part $v$ of the weak solution, but a bound for $\int_{\Omega} h^{2}|D v|^{2}$, where $\tilde{h}(z)=h \circ F^{-1}(z) \rightarrow \infty$ in most cases. Such a bound is obtained in section 3 for channels of type III. In section 6, the Green-function operator is used to analyse the far field and to obtain the foregoing results.

(iii) It is perhaps worth stressing that the boundary between types $I^{\prime}$ and III has a physical significance: for channels of type $I^{\prime}$, the pressure $p$ tends to a finite limit far upstream, and to another far downstream, while for channels of type III it tends to plus infinity far upstream and to minus infinity far downstream. This seems satisfactory because Definition 1.1 was dictated entirely by the need to bound terms resulting from the definition of weak solution in section 3 , and there the pressure $p$ does not appear. (The further conditions defining channels of type $I^{\prime}$ correspond to the transition, in section 5, from an averaged, or integral, treatment of the far field to a pointwise treatment, and these additional conditions seem natural in the light of examples in Appendix A.)

The two kinds of behaviour of $p$ are easily explained for channels $\Omega_{f}$, as in (1.6), with $k_{j}<1(j=1,2)$. For these, the distinctions between $h d \xi$ and $d x$, between $h(\xi, \eta)$ and $f(x)$, and between $\eta$ and $y / f(x)$, are not important at large distances, and a physicist would not hestitate to assert that the longitudinal velocity component $u_{1}$ has the asymptotic form $g(y / f(x)) / f(x)$ as $|x| \rightarrow \infty$, by conservation of mass (div $u=0$ ) and because $f(x)$ is the only 'natural length' at large distances. (As has been mentioned, we prove such a result in section 6 for channels of type III; it is also known to be true for certain rather special channels of type $I^{\prime}$, [12], [14]). Now the Navier-Stokes equations state that at a channel wall the tangential pressure gradient, essentially $-\hat{\partial} p / \partial x>0$ in the present case, balances the normal gradient of viscous shear stress, essentially $-\nu \partial^{2} u_{1} \mid \partial y^{2}$ here; hence $-\partial p / \partial x$ is asymptotically proportional to $1 / f(x)^{3}$. For a channel $\Omega_{f}$, this is integrable on $\mathbf{R}$ if and only if $k_{j}>\frac{1}{3}$, and then $\Omega_{f}$ is of type $\mathrm{I}^{\prime}$.

(iv) Various extensions of the results are immediate; we mention three.

(a) If $k_{1} \leqslant \frac{1}{3}$ and $k_{2} \geqslant 1$ in (1.6), then $\Omega_{f}$ does not belong to any of the types in Definition 1.l. It does not seem worth while to define channels of mixed type, but results for such channels can be inferred for $R<R_{0}$ from the theorems in this paper. A weak solution can be found by an easy modification of the velocity fields $g$ in section 3 to suit upstream and downstream geometries of different types; thereafter, what is proved 
about the solution far upstream is independent of conditions far downstream, and vice versa.

(b) Rotational force fields $f$ can be added to the right-hand side of (1.1), in which irrotational forces are already included, under the following restrictions: $f \in C^{\infty}\left(\bar{\Omega} \rightarrow \mathbf{R}^{2}\right)$; for channels of type I, $f$ has compact support in $\bar{\Omega}$; for channels of type II (or III), $|f(z)| \rightarrow 0$ sufficiently fast as $|z| \rightarrow \infty$. (For the analysis in section 5 of the far field in channels of type $I^{\prime}$, rapid decay of $f$ is not enough, because the maximum principle for $p+\frac{1}{2}|u|^{2}$ is essential.)

(c) To describe flow past obstacles within a channel, one may pose a boundary-value problem in the domain $\Omega^{\prime}=\Omega \backslash K$, where $K$ is a compact subset of a channel $\Omega$, with a boundary $\partial K$ that is of class $C^{\infty}$ when regarded as $\partial$ (int $K$ ), and has components $\Gamma_{1}, \ldots, \Gamma_{m}$, say. The additional boundary conditions can take the form

$$
\left.u\right|_{\Gamma_{j}}=a^{j}(x), \quad \text { where } \int_{\Gamma_{j}} a^{j} \cdot n d l=0, \quad j=1, \ldots, m,
$$

and where $a^{j} \in C^{\infty}\left(\Gamma_{,} \rightarrow \mathbf{R}^{2}\right)$. (For vector fields, superscript letters are mere labels, not exponents.) The conformal map $F$ for $\Omega$ can still be used, the inverse image $F^{-1}(K)$ being what it will; to construct a flux-carrying field $g$ satisfying all the boundary conditions, one merely adds terms which are familiar in Navier-Stokes theory for domains with compact boundaries, and whose contributions to the various estimates are easily bounded.

\section{Preliminaries}

\subsection{Further notation and terminology}

In what follows, symbols for functions composed with the conformal map $F$, or its inverse, will usually be abbreviated: for example, $h \circ F^{-1}$ and $u_{j} \circ F(j=1,2)$ will be shortened to $h$ and $u_{j}$; adjacent symbols $(\Omega, z, D$ or $S, \zeta, \nabla)$ will prevent confusion.

In the context of $\mathbf{R}^{N}$, integrals are with respect to $N$-dimensional Lebesgue measure unless the contrary is displayed. In particular,

$$
\int_{\Omega}()=\int_{\Omega}() d x d y, \quad \int_{S}()=\int_{S}() d \xi d \eta
$$

and, since the Jacobian $J(z / \zeta)=h^{2}$, while $|D f|=|\nabla f| / h$ for any smooth real-valued (scalar-valued) $f$,

$$
\int_{\Omega}()=\int_{S}() h^{2}, \int_{\Omega}|D f|^{2}=\int_{S}|\nabla f|^{2}
$$


In addition to the various notations for partial derivatives introduced already, including the suffices in (1.7), we shall have occasion to use $D^{\alpha}=D_{1}^{\alpha_{3}} \ldots D_{N}^{\alpha_{N}}$, where $\alpha_{1}, \ldots, \alpha_{N}$ are non-negative integers, and $\alpha=\left(\alpha_{1}, \ldots, \alpha_{N}\right)$ is a multi-index of order $|\alpha|=\alpha_{1}+\ldots+\alpha_{N}$. Similarly, $\nabla^{\alpha}=\nabla_{1}^{\alpha_{1}} \nabla_{2}^{\alpha_{2}}$. Let $U$ be an open set in $\mathbf{R}^{N}$; by the Sobolev space $W_{p}^{m}(U)$ we mean the real Banach space of functions $f: U \rightarrow \mathbf{R}$ such that $f$ and its generalized derivatives $D^{\alpha} f,|\alpha| \leqslant m$, belong to $L_{p}(U), p \geqslant 1$; the norm is

$$
\|f\|_{m, p, U}=\left\{\sum_{|\alpha| \leqslant m} \int_{U}\left|D^{\alpha} f\right|^{p}\right\}^{1 / p} .
$$

Note that $\|\cdot\|_{0, p, u}$ denotes the norm of $L_{p}(U)$. The closure in $W_{p}^{m}(U)$ of the set $C_{0}^{\infty}(U)$, of real-valued functions having derivatives of all orders and compact support in $U$, will be denoted by $\stackrel{\circ}{W}_{p}^{m}(U)$.

As elsewhere, $J(\Omega)=C_{0}^{\infty o s o l}\left(\Omega \rightarrow \mathbf{R}^{2}\right)$ denotes the set of infinitely differentiable vector fields $v=\left\langle v_{1}, v_{2}\right.$ ) that are solenoidal (div $v=D_{1} v_{1}+D_{2} v_{2}=0$ ) and have compact support in $\Omega$. Where convenient, functions in $J(\Omega)$ are defined to be zero in $\mathbf{R}^{2} \backslash \Omega$. We write

$$
D v: D w=\sum_{i, j=1}^{2}\left(D_{i} v_{j}\right)\left(D_{i} w_{j}\right), \quad|D v|^{2}=D v: D v
$$

for vector fields, and, when we transform to $S$, treat $v_{1}=v \cdot D x$ and $v_{2}=v \cdot D y$ like other scalars. Thus

$$
|\nabla v|^{2}=v_{1 \xi}^{2}+v_{1_{\eta}}^{2}+v_{2 \xi}^{2}+v_{2 \eta}^{2}
$$

For any smooth solenoidal velocity field $v$ defined on $\bar{\Omega}$, whether in $J(\Omega)$ or not, we can define a stream function $\psi$ by the line integral, along an arbitrary smooth simple arc in $\bar{\Omega}$ from a fixed reference point $a$,

$$
\psi\left(z_{0}\right)=\int_{a}^{z_{0}}\left(v_{1} d y-v_{2} d x\right)=\int v \cdot n d l
$$

The necessary and sufficient condition for this is div $v=0$, and then $v=\left(\psi_{y},-\psi_{x}\right)$. For the desired solution $u$ of $(1.1)$ to $(1.3)$, we write $u=\left(\Psi_{y},-\Psi_{x}\right)$; the flux condition (1.3b) becomes

$$
\left.\Psi\right|_{\Gamma_{-}}=c \text { and }\left.\Psi\right|_{\Gamma_{+}}=c+M
$$

where $c$ is a constant depending on the reference point of $\Psi$.

We define triple products by

$$
\{u, v, w\}=\int_{\Omega} u \cdot(v \cdot D) w
$$


whenever the vector fields $u, v$ and $w$ are such that the integral has meaning. If $\operatorname{div} v=0$, and $u \cdot(v \cdot n) w$ is zero on $\partial \Omega$ and $o(1 / h)$ on $\operatorname{arcs} \xi=$ const. in $\Omega$ as $|\xi| \rightarrow \infty$ (all these conditions need hold only in a generalized sense), then

$$
\{u, v, w\}=\sum_{i, j=1}^{2} \int_{\Omega} u_{i} D_{j}\left(v_{j} w_{i}\right)=-\sum_{i, j} \int_{\Omega} w_{i} v_{j} D_{j} u_{i}=-\{w, v, u\},
$$

so that

$$
\{w, v, w\}=0 \text {. }
$$

\subsection{The spaces $H(\Omega)$ and $V(\Omega)$}

The real Hilbert space $H(\Omega)$ is the completion of $J(\Omega)$ in the Dirichlet norm:

$$
\|v\|^{2}=\int_{\Omega}|D v|^{2}=\int_{S}|\nabla v|^{2}
$$

the inner product being $\langle v, w\rangle=\int_{\Omega} D v$ : $D w$. Here we have applied (2.1) to $v_{1}$ and $v_{2}$.

Functions in $H(\Omega)$ are weakly solenoidal ([23], [26]), and vanish on $\partial \Omega$ under the action of the trace operator: $H(\Omega) \rightarrow L_{2}(\partial \Omega)$ (more precisely, $L_{2}\left(\partial \Omega \rightarrow \mathbf{R}^{2}\right)$ ); for the construction of this operator we use the norm of $H(\Omega)$ and (2.8) below. (Trace operators are discussed in [1] and [24], for example.) For any fixed arc $\gamma$ as in (1.3b), we can use (2.6) or (2.8), and the fact that $h$ is bounded on $\gamma$, to construct a trace operator: $H(\Omega) \rightarrow L_{1}(\gamma)$, and it follows that velocity fields $v$ in $H(\Omega)$ carry no flux:

$$
\int_{\gamma} v \cdot n d l=0, \quad \forall v \in H(\Omega) .
$$

It suffices to prove this for $v \in J(\Omega)$, and we do so by means of a smooth simple closed curve $\Gamma$ such that $\gamma \subset \Gamma$, while $\Gamma \backslash \gamma$ lies outside the support supp $v$ of $v$. Then

because $\operatorname{div} v=0$.

$$
\int_{\gamma} v \cdot n d l=\int_{\Gamma} v \cdot n d l=0
$$

The following properties involve the form of $\Omega$ more closely; their analogues for bounded domains are stronger, and those for exterior domains in $\mathbf{R}^{2}$ are weaker.

Lемма 2.1. Let $\Omega$ be a channel. Then, for every $v \in H(\Omega)$,

$$
\begin{gathered}
\int_{\Omega} \frac{|v|^{2}}{h^{2}}=\int_{S}|v|^{2} \leqslant \frac{4}{\pi^{2}}\|v\|^{2}, \\
\int_{\Omega} \frac{|v|^{p}}{h^{2}}=\int_{S}|v|^{p} \leqslant K_{p}\|v\|^{p}, \quad 2 \leqslant p<\infty,
\end{gathered}
$$


where the constant $K_{p}$ depends only on $p$; also,

and

$$
\int_{\Omega} \frac{|v|^{2}}{h^{2}(\eta+1)^{2}}=\int_{S} \frac{|v|^{2}}{(\eta+1)^{2}} \leqslant 4\|v\|^{2}
$$

$$
\int_{-1}^{1}|v(\xi, \eta)|^{2} d \eta \rightarrow 0 \text { as }|\xi| \rightarrow \infty
$$

Proof. To prove (2.6) we integrate over $S$ first with respect to $\eta$, apply the celebrated one-dimensional inequality ([20], Theorem 257)

$$
\int_{0}^{l} f(t)^{2} d t \leqslant \frac{l^{2}}{\pi^{2}} \int_{0}^{l} f^{\prime}(t)^{2} d t, \quad \forall f \in \stackrel{\circ}{W}_{2}^{1}(0, l),
$$

and then integrate with respect to $\xi$. The inequality (2.7) is a result of Sobolev embedding for $\grave{W}_{2}^{1}(S)$ ([1], [24]). For (2.8) we can use another standard inequality ([20], Theorem 253), but a naive form of this is easily derived for functions in $J(\Omega)$ :

$$
\int_{-1}^{1} \frac{|v|^{2}}{(\eta+1)^{2}} d \eta=\int_{-1}^{1} \frac{1}{\eta+1} 2 v \cdot v_{\eta} d \eta \leqslant 2\left\{\int_{-1}^{1} \frac{|v|^{2}}{(\eta+1)^{2}} d \eta\right\}^{1 / 2}\left\{\int_{-1}^{1}\left|v_{\eta}\right|^{2} d \eta\right\}^{1 / 2}
$$

Dividing through by the first bracket on the right, squaring, integrating with respect to $\xi$ and extending the result to $H(\Omega)$ by continuity, we obtain (2.8). For (2.9), which refers to the $L_{2}$ trace on an are $\xi=$ const., we again take $v$ in $J(\Omega)$; then

$$
\left|v\left(\xi_{0}, \eta\right)\right|^{2}=-2 \int_{\xi_{0}}^{\infty} v \cdot v_{\xi} d \xi \leqslant \int_{\xi_{0}}^{\infty}\left(|v|^{2}+\left|v_{\xi}\right|^{2}\right) d \xi .
$$

Let $S_{0}=\left\{\zeta \in S \mid \xi>\xi_{0}\right\} ;$ then by $(2.10)$

$$
\int_{-1}^{1}\left|v\left(\xi_{0}, \eta\right)\right|^{2} d \eta \leqslant \int_{S_{0}}\left(|v|^{2}+\left|v_{\xi}\right|^{2}\right) \leqslant \int_{S_{0}}\left(\frac{4}{\pi^{2}}\left|v_{\eta}\right|^{2}+\left|v_{\xi}\right|^{2}\right)
$$

which tends to zero as $\xi_{0} \rightarrow \infty$. The result for $H(\Omega)$ then follows by continuity. Q.E.D.

If $v \in J(\Omega)$ and if in (2.2) the reference point $a \in \partial \Omega$, then $\psi \in C_{0}^{\infty}(\Omega)$. Conversely, to every $\psi \in C_{0}^{\infty}(\Omega)$ there corresponds an element $\left(\psi_{y},-\psi_{x}\right)$ of $J(\Omega)$. Let $w \in J(\Omega)$ have stream function $\chi$; then

$$
\langle v, w\rangle=\int_{\Omega}\left(\psi_{x x} \chi_{x x}+2 \psi_{x y} \chi_{x y}+\psi_{y y} \chi_{y y}\right)=\int_{\Omega}\left(D^{2} \psi\right)\left(D^{2} \chi\right)
$$

upon integration by parts of the middle term. We introduce 


$$
\langle\psi, \chi\rangle_{V(\Omega)}=\int_{\Omega}\left(D^{2} \psi\right)\left(D^{2} \chi\right)=\int_{S} \frac{1}{h^{2}}\left(\nabla^{2} \psi\right\rangle\left(\nabla^{2} \chi\right),
$$

where $\nabla^{2} \psi=\psi_{\xi \xi}+\psi_{\eta \eta}$, and define $V(\Omega)$ to be the completion of $C_{0}^{\infty}(\Omega)$ in the corresponding norm. The map $\psi \mapsto\left(\psi_{y},-\psi_{x}\right)$ defines an isometric isomorphism between $V(\Omega)$ and $H(\Omega)$.

When the local width of $\Omega$ is bounded, $V(\Omega)$ is equivalent to the Soboler space $\dot{W}_{2}^{2}(\Omega)$, because $\int_{\Omega}|D \psi|^{2}$ and $\int_{\Omega} \psi^{2}$ can then be bounded in terms of $\|\psi\|_{\nu(\Omega)}$ by means of (2.10), but in general $\dot{\circ}_{2}^{2}(\Omega)$ is a proper subset of $V(\Omega)$.

\subsection{Identities implied by the conformal map $F$}

In this section velocity fields need not belong to $H(\Omega)$, nor stream functions to $V(\Omega)$.

(i) When a stream function is given as a function of $\zeta$ (is given on $S$ ), as are those of the flux-carrying velocity fields $g$ in section 3 , it is useful to define not only $v=\left(\psi_{y},-\psi_{x}\right)$ but also

$$
\text { Then } \quad v_{1}+i v_{2}=e^{i \theta}\left(\hat{v}_{1}+i \hat{v}_{2}\right) \text {, }
$$

where $\theta$ is as in (1.5) and (1.7). Although at a fixed point in $\Omega$ the pairs $\left(v_{1}, v_{2}\right)$ and $\left(\hat{v}_{1}, \hat{v}_{2}\right)$ [more precisely, $\left.\left(\hat{v}_{1} \circ F^{-1}, \hat{v}_{2} \circ F^{-1}\right)\right]$ are representations of the same vector relative to different orthonormal bases (respectively $D x, D y$ and $h D \xi, h D \eta$ ), the fact that the second basis rotates as we pass to other points makes it safer to regard $\hat{v}=\left(\hat{v}_{1}, \hat{v}_{2}\right)$ always as an ordered pair, related to $v=\left(v_{1}, v_{2}\right)$ by (2.14). From (2.13) and (1.7) we find that

$$
\begin{aligned}
|\nabla \hat{v}|^{2} & =\hat{v}_{1 \xi}^{2}+\hat{v}_{1 \eta}^{2}+\hat{v}_{2 \eta}^{2}+\hat{v}_{2 \eta}^{2} \\
& =\frac{1}{h^{2}}\left\{\left(\psi_{\xi \xi}-x \psi_{\xi}\right)^{2}+\left(\psi_{\xi \eta}-\varkappa \psi_{\eta}\right)^{2}+\left(\psi_{\xi \eta}-\lambda \psi_{\xi}\right)^{2}+\left(\psi_{\eta \eta}-\lambda \psi_{\eta}\right)^{2}\right\} .
\end{aligned}
$$

For computations like the next one, the $\mathrm{C}^{2}$-valued function $\nabla\left(v_{1}+i v_{2}\right)=\left(v_{1 \xi}+i v_{2 \xi}, v_{1 \eta}+i v_{2 \eta}\right)$ is more convenient, because of the complex scalar $e^{i \theta}$, than the $\mathbf{R}^{4}$-valued function $\nabla\left(v_{1}, v_{2}\right)$; the norms are, of course, equal. Thus (2.14) and (1.7) yield

$$
\begin{aligned}
|\nabla v|^{2} & =\left(\hat{v}_{1 \xi}+\lambda \hat{v}_{2}\right)^{2}+\left(\hat{v}_{2 \xi}-\lambda \hat{v}_{1}\right)^{2}+\left(\hat{v}_{1 \eta}-\varkappa \hat{v}_{2}\right)^{2}+\left(\hat{v}_{2 \eta}+\varkappa \hat{v}_{1}\right)^{2} \\
& =\frac{1}{h^{2}}\left\{\left(\psi_{\xi \xi}-\varkappa \psi_{\xi}+\lambda \psi_{\eta}\right)^{2}+2\left(\psi_{\xi \eta}-\varkappa \psi_{\eta}-\lambda \psi_{\xi}\right)^{2}+\left(\psi_{\eta \eta}-\lambda \psi_{\eta}+\varkappa \psi_{\xi}\right)^{2}\right\} .
\end{aligned}
$$

(ii) We shall use, at least once, each of the following forms of the Navier-Stokes equation (1.1). With $u=\left(\Psi_{y},-\Psi_{x}\right)$, the vorticity $\omega$ is defined by

$$
\omega=u_{2 x}-u_{1 y}=-D^{2} \Psi=-\frac{1}{h^{2}} \nabla^{2} \Psi^{2}
$$


In terms of this, and because $\operatorname{div} u=0$, (1.1) may be written

$$
\begin{array}{r}
\nu \omega_{y}-\left(u_{1} u_{2 y}-u_{2} u_{1 y}\right)=-p_{x} \\
-\nu \omega_{x}+\left(u_{1} u_{2 x}-u_{2} u_{1 x}\right)=-p_{y} .
\end{array}
$$

We obtain the first (second) of the next pair upon multiplying (2.16a) by $h \cos \theta$ (by $-h \sin \theta$ ), multiplying $(2.16 \mathrm{~b})$ by $h \sin \theta$ (by $h \cos \theta$ ), and adding; there results

$$
\begin{aligned}
\nu \omega_{\eta}-\left(u_{1} u_{2 \eta}-u_{2} u_{1 \eta}\right) & =-p_{\xi}, \\
-v \omega_{\xi}+\left(u_{1} u_{2 \xi}-u_{2} u_{1 \xi}\right) & =-p_{\eta} .
\end{aligned}
$$

Now observe that $u_{1} u_{2 \eta}-u_{2} u_{1 \eta}=\operatorname{Im}\left(u_{1}-i u_{2}\right)\left(u_{1 \eta}+i u_{2 \eta}\right)$. Using (2.14), we obtain

$$
\begin{aligned}
& \nu \omega_{\eta}-\hat{u}_{1}\left(\hat{u}_{2 \eta}+x \hat{u}_{1}\right)+\hat{u}_{2}\left(\hat{u}_{1 \eta}-x \hat{u}_{2}\right)=-p_{\xi}, \\
& -v \omega_{\xi}+\hat{u}_{1}\left(\hat{u}_{2 \xi}-\lambda \hat{u}_{1}\right)-\hat{u}_{2}\left(\hat{u}_{1 \xi}+\lambda \hat{u}_{2}\right)=-p_{\eta} .
\end{aligned}
$$

(iii) As before, let $v$ and $w$ have respective stream functions $\psi$ and $\chi$. Multiplying (2.18a) by $\hat{v}_{1}$ and $(2.18 \mathrm{~b})$ by $\hat{v}_{2}$, and recalling that $(2.18)$ corresponds to $h$ times (1.1), we infer that

$$
\begin{gathered}
v \cdot D^{2} w=\frac{1}{h^{4}}\left\{\psi_{\eta}\left(\frac{\partial}{\partial \eta}-2 \lambda\right) \nabla^{2} \chi+\psi_{\xi}\left(\frac{\partial}{\partial \xi}-2 \chi\right) \nabla^{2} \chi\right\} \\
v \cdot(w \cdot D) w=\frac{1}{h^{4}} \psi_{\eta}\left\{\chi_{\eta} \chi_{\xi \eta}-\chi_{\xi} \chi_{\eta \eta}-\chi\left(\chi_{\xi}^{2}+\chi_{\eta}^{2}\right)\right\}+\frac{1}{h^{4}} \psi_{\xi}\left\{\chi_{\eta} \chi_{\xi \xi}-\chi_{\xi} \chi_{\xi \eta}+\lambda\left(\chi_{\xi}^{2}+\chi_{\eta}^{2}\right)\right\} .
\end{gathered}
$$

(iv) If we take the curl of (1.1) (or eliminate $p$ from (2.16) by cross-differentiation), there results the vorticity equation, four forms of which are

$$
\begin{gathered}
\nu D^{2} \omega-u \cdot D \omega=0 \\
\left\{v D^{2}-\left(\Psi_{y} \frac{\partial}{\partial x}-\Psi_{x} \frac{\partial}{\partial y}\right)\right\} D^{2} \Psi=0 \\
\frac{1}{h^{2}}\left\{\nu \nabla^{2}-\left(\Psi_{\eta} \frac{\partial}{\partial \xi}-\Psi_{\xi} \frac{\partial}{\partial \eta}\right)\right\}\left(\frac{\nabla^{2} \Psi}{h^{2}}\right)=0 \\
\frac{1}{h^{4}}\left\{\nu\left[\nabla^{2}-4\left(x \frac{\partial}{\partial \xi}+\lambda \frac{\partial}{\partial \eta}\right)+4\left(x^{2}+\lambda^{2}\right)\right]-\left[\Psi_{\eta}\left(\frac{\partial}{\partial \xi}-2 x\right)-\Psi_{\xi}\left(\frac{\partial}{\partial \eta}-2 \lambda\right)\right]\right\} \nabla^{2} \Psi=0
\end{gathered}
$$


The fact that $\nabla^{2}(\log h)=0$ (that $\varkappa_{\xi}+\lambda_{\eta}=0$ ), which has been used here, also allows us to obtain results in section 3 without assumptions about second derivatives of $h$.

\subsection{Two properties of channels of type II}

We recall that $\nabla(\log h)=(x, \lambda)$. Neither of the following results uses the second condition, $x / h$ and $\lambda / h \in L_{2}(S)$, defining channels of type II; that will be used only in Lemma 3.3.

LEMMA 2.2. If $\lambda(\xi, \eta) \rightarrow 0$ as $|\xi| \rightarrow \infty$, uniformly over $\eta$, then the limit inferior

$$
\underline{\lim }_{|\zeta| \rightarrow \infty} h(\zeta) \geqslant \frac{1}{2} \operatorname{dist}\left(\Gamma_{-}, \Gamma_{+}\right)>0
$$

Proof. Since $\lambda=h_{\eta} / h$,

$$
\log \frac{h\left(\xi, \eta_{2}\right)}{h\left(\xi, \eta_{1}\right)}=\int_{\eta_{1}}^{\eta_{2}} \lambda(\xi, \eta) d \eta \rightarrow 0
$$

uniformly over $\eta_{1}$ and $\eta_{2} \in[-1,1]$, as $|\xi| \rightarrow \infty$, and so

$$
\sup _{\eta_{\mathrm{L}}, \eta_{2} \in[-1,1]} \frac{h\left(\xi, \eta_{2}\right)}{h\left(\xi, \eta_{1}\right)} \rightarrow 1
$$

Then

$$
\begin{aligned}
\operatorname{dist}\left(\Gamma_{-}, \Gamma_{+}\right) & \leqslant|z(\xi, 1)-z(\xi,-1)| \leqslant \int_{-1}^{1} h(\xi, \eta) d \eta \\
& \leqslant 2\{1+o(1)\} \min _{\eta \in[-1,1]} h(\xi, \eta)
\end{aligned}
$$

and a $\lim h(\zeta)$ smaller than that in (2.22) would contradict this.

Q.E.D.

Lемм 2.3. Let $\Omega$ be a channel such that $\varkappa(\xi, \eta) \rightarrow 0$ and $\lambda(\xi, \eta) \rightarrow 0$ as $|\xi| \rightarrow \infty$, uniformly over $\eta$. Define

$$
S_{n}=\{\zeta \in S|| \xi \mid>n\}, \quad \alpha(n)=\sup _{|\xi|>n}\{|\varkappa(\zeta)|,|\lambda(\zeta)|\},
$$

and let $n$ be so large that $\alpha(n) \leqslant \pi / 4$. Then, for all $\psi \in V(\Omega)$,

$$
\left.\begin{array}{l}
\int_{S_{n}}\left(\psi_{\xi} / h\right)^{2} \leqslant \frac{16}{\pi^{2}} \int_{S_{n}}\left(\psi_{\xi \eta} / h\right)^{2}, \quad \int_{S_{n}}\left(\psi_{\eta} / h\right)^{2} \leqslant \frac{16}{\pi^{2}} \int_{S_{n}}\left(\psi_{\eta \eta} / h\right)^{2}, \\
\int_{S_{n}}(\psi / h)^{2} \leqslant \frac{256}{\pi^{4}} \int_{S_{n}}\left(\psi_{\eta \eta} / h\right)^{2} .
\end{array}\right\}
$$

7-792901 Acta mathematica 144. Imprimé le 13 Juin 1980 
This implies that

$$
\{1-k \alpha(n)\} \int_{S_{n}} \frac{1}{h^{2}} \psi_{(\zeta \zeta)}^{2} \leqslant \int_{S_{n}}|\nabla v|^{2} \leqslant\{1+k \alpha(n)\} \int_{S_{n}} \frac{1}{h^{2}} \psi_{(\zeta \zeta)}^{2},
$$

where

$$
\psi_{(\zeta \zeta)}^{2}=\psi_{\xi \xi}^{2}+2 \psi_{\xi \eta}^{2}+\psi_{\eta \eta}^{2}
$$

$v=\left(\psi_{y},-\psi_{x}\right)$ and $k$ is an absolute constant (independent of $\Omega, n$ and $\psi$ ).

Proof. In this proof $c=4 / \pi^{2}, \alpha=\alpha(n)$ and all integrals are over $S_{n}$; we use repeatedly (2.10), with $t=\eta+1$ and $l=2$, and the inequality $2 a b \leqslant a^{2}+b^{2}$. Thus

$$
\int\left(\psi_{\xi} / h\right)^{2} \leqslant c \int\left(\psi_{\xi \eta}-\lambda \psi_{\xi}\right)^{2} / h^{2} \leqslant 2 c \int\left(\psi_{\xi \eta}^{2}+\lambda^{2} \psi_{\xi}^{2}\right) / h^{2}
$$

and, since $\lambda^{2} \leqslant \alpha^{2}$, there results

$$
\int\left(\psi_{\xi} / h\right)^{2} \leqslant \frac{2 c}{1-2 c \alpha^{2}} \int\left(\psi_{\xi \eta} / h\right)^{2}
$$

which is the first inequality of (2.23); note that $\alpha \leqslant \pi / 4$ makes $1-2 c \alpha^{2} \geqslant \frac{1}{2}$. The other two are proved in the same way.

For (2.24), we refer to the formula (2.15) for $|\nabla v|^{2}$ in terms of $\psi$. The terms $\psi_{(\zeta \zeta)}^{2} / h^{2}$ are left as they are; for the others, we use $|\varkappa(\zeta)| \leqslant \alpha, \varkappa(\zeta)^{2} \leqslant \alpha \pi / 4,(2.23)$ and any one of a number of algebric inequalities for product terms.

Q.E.D.

\section{Existence of weak solutions}

\subsection{A priori bounds}

Definition 3.1. For any channel $\Omega$, a vector field will be called a flux carrier if it belongs to $C^{\infty}\left(\bar{\Omega} \rightarrow \mathbf{R}^{2}\right)$ and satisfies (1.2) to (1.4).(1)

A velocity field $u=g+v$ is a weak (or generalized) solution of (1.1) to (1.4) if $g$ is a flux carrier, $v \in H(\Omega)$ and

or, equivalently,

$$
v \int_{\Omega} D w: D u+\{w, u, u\}=0, \quad \forall w \in J(\Omega)
$$

$$
v\langle w, v\rangle+\{w, g+v, v\}+\{w, v, g\}=-v \int_{\Omega} D w: D g-\{w, g, g\}
$$

for all $w \in J(\Omega)$.

(1) Regarding (1.4) we recall the remarks made there. 

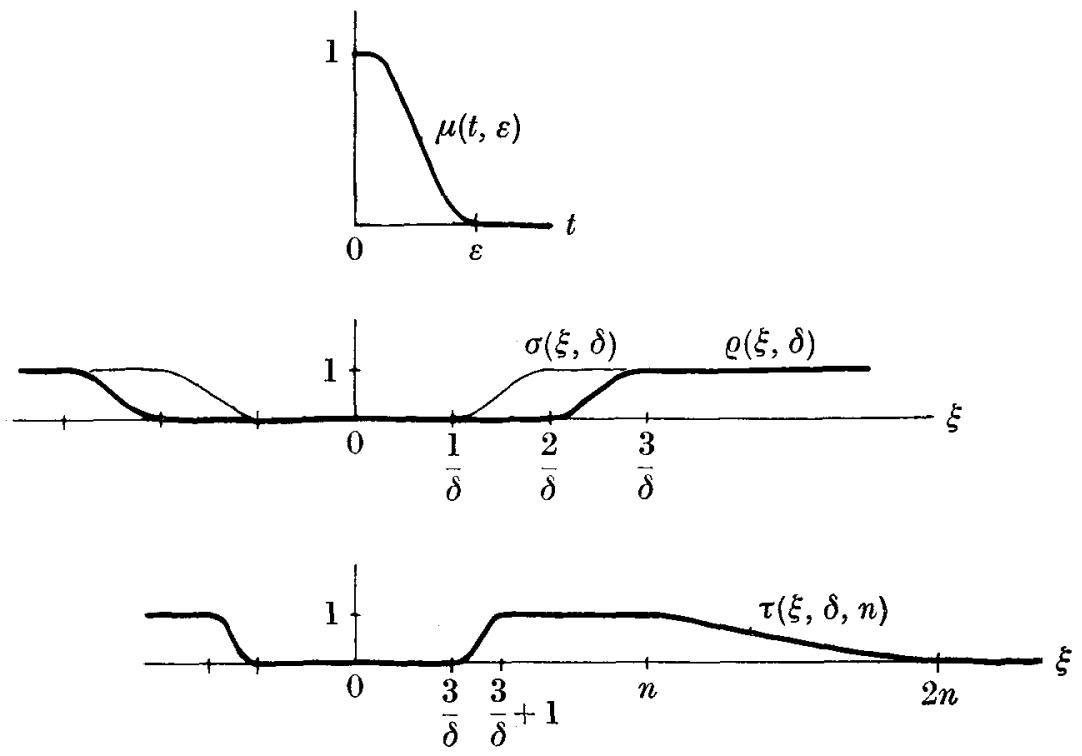

Fig. 2. The four mollifiers used in section 3 .

The condition $v \in H(\Omega)$ implies weak forms of (1.2) to (1.4); for the weak form of (1.4) we refer to (2.9). Eq. (3.1) correspond to (1.1): if $u$ is a classical solution, we obtain (3.1) upon multiplying (1.1) by any $w \in J(\Omega)$ and integrating by parts; the converse, that (3.1) implies (1.1), will be shown in section 4.

LEMMA 3.2. For channels $\Omega$ of type $I$ and any Reynolds number $M / v \in(0, \infty)$, there exists a flux carrier $g$ such that, if $v \in H(\Omega)$ and

$$
v\|v\|^{2}-\{g, v, v\}=-v \int_{\Omega} D v: D g-\{v, g, g\},
$$

then

$$
\|v\| \leqslant \text { const. }
$$

where the constant depends only on the data $\Omega, \nu$ and $M$.

Proof. (i) The velocity $g$ will be that of two jets, each near a channel wall, that are narrow relative to the local channel width and are fast wherever $h(\xi, \eta)$ is not large.

For any $\varepsilon>0$, let $\mu(., \varepsilon) \in C^{\infty}([0, \infty) \rightarrow[0,1])$ be the usual mollifier (Fig. 2) used in Navier-Stokes problems for extending boundary-value functions ([4], p. 483; [9], p. 129; [13], p. T9; [16], p. 72): $\mu(t, \varepsilon)=1$ at, and sufficiently near, $t=0 ; \mu(t, \varepsilon)=0$ for $t \geqslant \varepsilon$; and for $t>0$ 


$$
\mu(t, \varepsilon) \leqslant \varepsilon / t, \quad 0 \leqslant-\mu^{\prime}(t, \varepsilon) \leqslant \varepsilon / t,
$$

where $\mu^{\prime}$ denotes the derivative with respect to $t$.

We define $g=\left(G_{y},-G_{x}\right)$, where the stream function

$$
G(\zeta, \varepsilon)=-\frac{1}{2} M\{\mu(\eta+1, \varepsilon)-\mu(1-\eta, \varepsilon)\}
$$

and where $\varepsilon<1$ will be chosen presently. In view of the form (2.3) of the flux condition $(1.3 \mathrm{~b})$, and the formula [recall (2.13) and (2.14)]

$$
\hat{g}_{1}(\zeta, \varepsilon)=-\frac{1}{2} \frac{M}{h(\zeta)}\left\{\mu^{\prime}(\eta+1, \varepsilon)+\mu^{\prime}(1-\eta, \varepsilon)\right\}, \quad \hat{g}_{2}=0
$$

it is clear that $g$ satisfies (1.2) and (1.3), and (1.4) in the sense explained there.

(ii) Since $|g|=\hat{g}_{1}$, we obtain from (3.4) and (3.5)

$$
\begin{aligned}
\{g, v, v\} & =\int_{\Omega} g \cdot(v \cdot D) v \\
& \leqslant \frac{1}{2} M \varepsilon \int_{\Omega} \frac{1}{h}\left(\frac{|v|}{\eta+1}+\frac{|v|}{1-\eta}\right)|D v| \\
& \leqslant 2 M \varepsilon\|v\|^{2}
\end{aligned}
$$

by the Schwarz inequality and (2.8). We choose $\varepsilon=\varepsilon_{0}$ in $(0,1)$ such that $2 M \varepsilon_{0} \leqslant \frac{1}{2} \nu$, and let $g=g\left(., \varepsilon_{0}\right)$ henceforth; then the left side of (3.2) is not less than $\frac{1}{2} v\|v\|^{2}$.

(iii) With $\varepsilon$ now fixed, $G_{\eta}$ and $G_{\eta \eta}$ are uniformly bounded pointwise; by (2.15),

$$
\begin{aligned}
\int_{S}|\nabla g|^{2} & =\int_{S} \frac{1}{h^{2}}\left\{\left(\lambda G_{\eta}\right)^{2}+2\left(\varkappa G_{\eta}\right)^{2}+\left(G_{\eta \eta}-\lambda G_{\eta}\right)^{2}\right\} \\
& \leqslant \text { const. } \int_{S} \frac{1}{h^{2}}\left\{1+\varkappa^{2}+\lambda^{2}\right\}=\text { const. }\left\|\frac{1}{h}\right\|_{1,2, S}^{2},
\end{aligned}
$$

where $1 / h \in W_{2}^{1}(S)$ because $\Omega$ is of type I. Thus $|\nabla g| \in L_{2}(S)$ and so $|D g| \in L_{2}(\Omega)$. Accordingly,

$$
\begin{aligned}
& -v \int_{\Omega} D v: D g \leqslant v\|v\|\|D g\|_{0,2, \Omega}=c_{1}\|v\|, \text { say, } \\
& -\{v, g, g\} \leqslant \text { const. } \int_{\Omega} \frac{|v|}{h}|D g| \leqslant \text { const. }\|v\|=c_{2}\|v\|, \text { say, }
\end{aligned}
$$

where we have used the Schwarz inequality and (2.6). It follows that $\|v\| \leqslant 2\left(c_{1}+c_{2}\right) / \nu$. 
LEM M 3.3. Let $W=W(S)$ be the set $\dot{W}_{2}^{2}(S)$ normed by

$$
\|\chi\|_{W}^{2}=\int_{S}\left(\nabla^{2} \chi\right)^{2}
$$

so that $W$ is equivalent to $\stackrel{\circ}{W}_{2}^{2}(S)$, and define

$$
v_{0}=\sup _{\chi \in W \backslash\{0\}} \int_{S} Q_{\eta}\left(\chi_{\eta} \chi_{\xi \eta}-\chi_{\xi} \chi_{\eta \eta}\right) /\|\chi\|_{W}^{2},
$$

where $Q(\eta)=\frac{3}{4} M\left(\eta-\frac{1}{3} \eta^{3}\right)$ is the stream function of Poiseuille flow in S. For channels of type II and $\boldsymbol{\nu}>v_{0}$, there exists a flux carrier $g$, different at large distances from that in Lemma 3.2 , such that (3.2) implies (3.3) for any $v \in H(\Omega)$.

Remark. In [5], careful analysis of the functional whose supremum appears above, followed by numerical computation, shows that $\nu_{0}=M / 116.5$. If only odd functions of $\eta$ are admitted, as is legitimate when one seeks symmetrical velocity fields in a symmetrical channel, then $v_{0}=M / 194.6$.

Proof of Lemma 3.3. (i) We construct $g$ to be as in Lemma 3.2 on a certain compact subset of $\bar{\Omega}$ and to be a slightly distorted Poiseuille velocity at large distances. To this end, we use, in addition to the earlier mollifier $\mu$, a second one $\varrho(., \delta) \in C^{\infty}(\mathbf{R} \rightarrow[0,1])$ shown in Fig. 2 and such that $\varrho(\xi, \delta)=0$ for $|\xi| \leqslant 2 / \delta$ and $\varrho(\xi, \delta)=1$ for $|\xi| \geqslant 3 / \delta$; also, $\left|\varrho^{\prime}(\xi, \delta)\right| \leqslant$ const. $\delta$ and $\left|\varrho^{\prime \prime}(\xi, \delta)\right| \leqslant$ const. $\delta^{2}$ for $2 / \delta<|\xi|<3 / \delta$, dashes denoting derivatives with respect to $\xi$. We set $g=\left(G_{y},-G_{x}\right)$ and define

$$
\left.\begin{array}{rl}
G=A+B, \quad A(\zeta, \varepsilon, \delta) & =-\frac{1}{2} M\{\mu(\eta+1, \varepsilon)-\mu(1-\eta, \varepsilon)\}\{1-\varrho(\xi, \delta)\}, \\
B(\zeta, \delta) & =Q(\eta) \varrho(\xi, \delta) .
\end{array}\right\}
$$

Further, $a=\left(A_{y},-A_{x}\right)$ and $b=\left(B_{y},-B_{x}\right)$. As before, $g$ satisfies (1.2) to (1.4), and $\varepsilon, \delta$ will be assigned positive values in due course.

(ii) Provided that $\left|\varrho^{\prime}\right| \leqslant 1$, we have $|a|$ at most $2 \frac{1}{2}$ times the bound for the $|g|$ of Lemma 3.2, and so (3.6) implies that

$$
\{a, v, v\} \leqslant 2^{3 / 2} M \varepsilon\|v\|^{2}
$$

Let $v=\left(\psi_{y},-\psi_{x}\right)$, then by $(2.20)$

$$
\{b, v, v\}=\int_{S} \frac{\varrho}{h^{2}} Q_{\eta}\left(\psi_{\eta} \psi_{\xi \eta}-\psi_{\xi} \psi_{\eta \eta}\right)+R_{1}
$$


where remainder terms $R_{j}$, containing the functions $\varrho^{\prime}, x$ and $\lambda$ that are small on supp $\varrho(., \delta)$ when $\delta$ is small, are recorded in Appendix B. Let

$$
v_{*}(\delta)=\sup _{x \in W \backslash\{0\}} \int_{S} \varrho Q_{\eta}\left(\chi_{\eta} \chi_{\xi \eta}-\chi_{\xi} \chi_{\eta \eta}\right) /\|\chi\|_{W}^{2}
$$

it is proved in [4], p. 510, that $\nu_{*}(\delta) \rightarrow \nu_{0}$ as $\delta \rightarrow 0$, and we plan to use $\nu_{*}(\delta)$ to bound $\{b, v, v\}$. Introducing a third mollifier $\sigma(\xi, \delta)$ (Fig. 2), even in $\xi$ and such that $\sigma(\xi, \delta)=$ $\varrho(\xi+1 / \delta, \delta)$ for $\xi \geqslant 0$, we define $\phi=\sigma \psi / h$ and $f=\sigma \psi$.

Assume for the moment that $\psi \in C_{0}^{\infty}(S)$; then $\phi \in C_{0}^{\infty}(S)$ and

$$
\|\phi\|_{W}^{2}=\int_{S} \frac{1}{h^{2}}\left\{\nabla^{2} f-2\left(x f_{\xi}+\lambda f_{\eta}\right)+\left(\varkappa^{2}+\lambda^{2}\right) f\right\}^{2},
$$

and

$$
\int_{S} \frac{1}{h^{2}}\left(\nabla^{2} f\right)^{2}=\int_{S} \frac{1}{h^{2}} f_{(\zeta \zeta)}^{2}+\int_{S} \frac{1}{h^{2}}\left(4 x f_{\xi} f_{\eta \eta} \cdots 4 \lambda f_{\xi} f_{\xi \eta}\right)
$$

by integration by parts; here $(\cdot)_{(5)}$ is as in Lemma 2.3. Let $\alpha$ bound $x$ and $\lambda$ as in that lemma; we apply $2 a b \leqslant a^{2}+b^{2}$ (say) and (2.23) repeatedly, first to the factors of $\varkappa, \lambda, x \lambda, \ldots$ above and then to the $\sigma^{\prime}$ and $\sigma^{\prime \prime}$ terms arising from $f=\sigma \psi$. There results

$$
\begin{aligned}
\|\phi\|_{W}^{2} & \leqslant(1+\text { const. } \alpha(1 / \delta)+\text { const. } \delta) \int_{|\xi|>1 / \delta} \frac{1}{h^{2}} \psi_{(\zeta \zeta)}^{2} \\
& \leqslant\left\{1+\beta_{0}(\delta)\right\}\|v\|^{2}
\end{aligned}
$$

where $\beta_{0}(\delta) \rightarrow 0$ as $\delta \rightarrow 0$, and similarly for other $\beta_{j}$ below. This inequality now extends to $v \in H(\Omega)$. The mollifier $\sigma$ plays no further part, because its value on supp $\varrho$ is one.

If we apply (3.10) as it stands to $\phi=\sigma \psi / h$, second derivatives of $h$ will appear; therefore we integrate by parts. By (3.9),

$$
\{b, v, v\}=\int_{S} \frac{\varrho}{h^{2}} Q_{\eta \eta} \psi_{\xi} \psi_{\eta}+R_{1}+R_{2}
$$

Also,

$$
\begin{aligned}
\int_{S} \varrho Q_{\eta}\left(\phi_{\eta} \phi_{\xi \eta}-\phi_{\xi} \phi_{\eta \eta}\right) & =\int_{S} \varrho Q_{\eta \eta} \phi_{\xi} \phi_{\eta}-\int_{S} \varrho^{\prime} Q_{\eta} \phi_{\eta}^{2} \\
& =\int_{S} \frac{\varrho}{h^{2}} Q_{\eta \eta} \psi_{\xi} \psi_{\eta}-R_{3},
\end{aligned}
$$

where $R_{j} \leqslant \beta_{j}(\delta)\|v\|^{2}, j=1$ to 3 , by inspection of these terms (Appendix B) and Lemma 2.3. Now using (3.10) and (3.11), and adding the estimate (3.8), we have 


$$
\begin{aligned}
\{g, v, v\} & \leqslant\left\{\text { const. } \varepsilon+\nu_{*}(\delta)\left[1+\beta_{0}(\delta)\right]+\sum_{j=1}^{3} \beta_{j}(\delta)\right\}\|v\|^{2} \\
& \leqslant \frac{1}{2}\left(\nu+v_{0}\right)\|v\|^{2}
\end{aligned}
$$

if we choose $\varepsilon=\varepsilon_{0}$ and $\delta=\delta_{0}$ to be sufficiently small; henceforth $g=g\left(., \varepsilon_{0}, \delta_{0}\right)$.

(iii) With $\varepsilon$ and $\delta$ now fixed, not only are the values of $G$ and each of its $\zeta$-derivatives bounded, but so are the set, say $S^{\prime}$, on which $|\xi|<3 / \delta_{0}$ and $G \neq Q$, and the values of $h$ on that set. Hence the Dirichlet integral of $v$ over $\Omega^{\prime}=F\left(S^{\prime}\right)$ is equivalent, by Lemma 2.1, to the square of the norm of $v$ in $W_{2}^{1}\left(\Omega^{\prime} \rightarrow \mathbf{R}^{2}\right)$, and the contribution of $\Omega^{\prime}$ to the right-hand side of (3.2) is at most $K_{1}\|v\|$, for some (possibly enormous) constant $K_{1}$.

Let $S^{\prime \prime}=S \backslash S^{\prime}$ and $\Omega^{\prime \prime}=F\left(S^{\prime \prime}\right)$; it remains to bound the contribution of $\Omega^{\prime \prime}$ to the right-hand side of (3.2). It is only for this that we use the second condition, $x / h$ and $\lambda / h \in L_{2}(S)$, in the definition of type II; because of Lemma 2.3 , this condition allows us to bound integrals over $S^{\prime \prime}$ of $x \nabla^{\gamma} \psi / h^{2}$ and $\lambda \nabla^{\gamma} \psi / h^{2},|\gamma| \leqslant 2$, by the Schwarz inequality. We shall also use the fact that $Q_{\eta \eta \eta}=-3 M / 2$. By (2.19) and (2.20),

$$
\begin{aligned}
v \int_{\Omega^{\prime \prime}} v \cdot D^{2} g & =v \int_{S^{\prime \prime}} \frac{1}{h^{2}}\left\{\psi_{\eta}\left(Q_{\eta \eta}-2 \lambda Q_{\eta \eta}\right)-\psi_{\xi} 2 \varkappa Q_{\eta \eta}\right\} \\
& =-v\left\{3 M \int_{S^{\prime \prime}} \frac{\lambda \psi}{h^{2}}+\int_{S^{\prime}} \frac{1}{h^{2}}\left(2 \lambda \psi_{\eta}+2 \varkappa \psi_{\xi}\right) Q_{\eta \eta}\right\} \\
& \leqslant \text { const. }\|v\|=k_{2}\|v\|, \text { say; } \\
-\{v, g, g\}_{\Omega^{\prime \prime}} & =\int_{S^{\prime \prime}} \frac{1}{h^{2}}\left(\psi_{\eta} x-\psi_{\xi} \lambda\right) Q_{\eta}^{2} \\
& \leqslant \text { const. }\|v\|=k_{3}\|v\|, \text { say. }
\end{aligned}
$$

Thus (3.2) implies that $\|v\| \leqslant 2\left(K_{1}+k_{2}+k_{3}\right) /\left(v-v_{0}\right)$.

Q.E.D.

\subsection{Existence of weak solutions}

ТнЕов. 3.4. The problem (1.1) to (1.4) has a weak solution $u$ for each Reynolds number $R=M / v \in(0, \infty)$ if $\Omega$ is of type $I$, and for $R<M / \nu_{0}$ if $\Omega$ is of type $I I$ ( $v_{0}$ being as in Lemma 3.3).

Proof. Let $\left\{\Omega_{m}\right\}, m=1,2, \ldots$, be an expanding sequence of simply connected, bounded subdomains of $\Omega$ such that $\Omega_{m} \rightarrow \Omega$ as $m \rightarrow \infty$ and $\partial \Omega_{m}$ is of class $C^{\circ}$. We may, and shall, suppose that $\Omega_{m}=F\left(S_{m}\right)$, where $S_{m}$ is as in Fig. 3. Consider the problem of finding a solution $\left(u^{m}, p_{m}\right)$ of $(1.1)$ and (1.2) such that $\left.u^{m}\right|_{\partial \Omega_{m}}=g$; this implies that $u^{m}=0$ on $\partial \Omega \cap \partial \Omega_{m}$ and that $u^{m}$ satisfies $(1.3 \mathrm{~b})$ for any $\operatorname{arc} \gamma$, in $\bar{\Omega}_{m}$, directed from a point of 


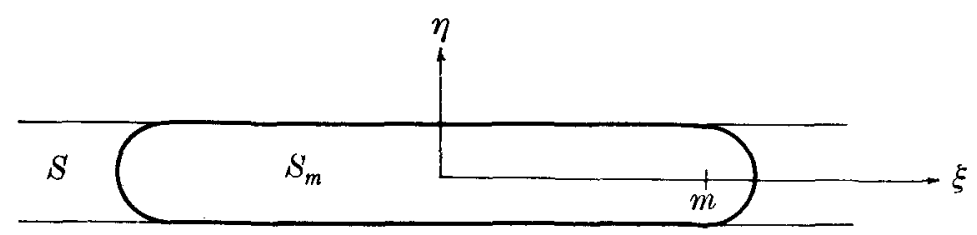

Fig. 3. A member of the sequence $\left\{S_{m}\right\}$ used in the proof of Theorem 3.4.

$\Gamma_{-} \cap \partial \Omega_{m}$ to one of $\Gamma_{+} \cap \partial \Omega_{m}$ It is known ([9], [16], [23], [26]) that this problem has a weak solution $u^{m}=g+v^{m}$, defined as above but with $\Omega_{m}$ replacing $\Omega$; in particular, $v^{m} \in H\left(\Omega_{m}\right)$. Since (for fixed $m$ ) $\Omega_{m}$ is bounded, (2.7) shows that $H\left(\Omega_{m}\right)$ is embedded in $L_{p}\left(\Omega_{m} \rightarrow \mathbf{R}^{2}\right.$ ) for all $p \in[1, \infty)$, and this allows the analogue of (3.1) to be extended to all test functions $w \in H\left(\Omega_{m}\right)$. For the triple products in this analogue, the integration by parts yielding (2.4) and (2.5) is valid; choosing $w=v^{m}$, we obtain

$$
v\left\|v^{m}\right\|^{2}-\left\{g, v^{m}, v^{m}\right\}=-v \int_{\Omega} D v^{m}: D g-\left\{v^{m}, g, g\right\},
$$

where $v^{m} \in H(\Omega)$ if we set $v^{m}(z)=0$ outside $\Omega_{m}$. Thus each $v^{m}$ satisfies (3.2).

By Lemmas 3.2 and 3.3, $\left\|v^{m}\right\|$ is bounded independently of $m$. Hence there exist a subsequence $\left\{v^{m_{n}}\right\}$ and an element $v \in H(\Omega)$ such that $v^{m_{n} \rightarrow v}$ weakly in $H(\Omega)$ as $n \rightarrow \infty$. The verification of $(3.1 \mathrm{~b})$, from the corresponding equations for the $v^{m_{n}}$, is straightforward because the test functions $w$ have compact support. For any given $w \in J(\Omega)$ we have supp $w \subset \Omega_{k}$ for some $k$, so that $v^{m_{n}}$ satisfies $(3.1 \mathrm{~b})$ for that $w$ if $m_{n} \geqslant k$. With this same $w$ fixed, the terms on the left of $(3.1 \mathrm{~b})$ that are linear in $v$ define a bounded linear functional, say $f_{w, g}: H(\Omega) \rightarrow \mathbf{R}$, and then $f_{w, g}\left(v^{m_{n}}\right) \rightarrow f_{w, g}(v)$ as $n \rightarrow \infty$, by the definition of weak convergence. Using the compact support of $w$ once more, we write the non-linear term as $-\{v, v, w\}$; it is then easily handled by means of the triple Hölder inequality because $\|v\| \leqslant \underline{\lim }\left\|v^{m_{n}}\right\| \leqslant$ const. and because the embedding of $W_{2}^{1}\left(\Omega_{k} \rightarrow \mathbf{R}^{2}\right)$ in $L_{p}\left(\Omega_{k} \rightarrow \mathbf{R}^{2}\right)$ is compact for all $p \in[1, \infty)$.

Q.E.D.

\subsection{An a posteriori estimate for weak solutions in channels of type III}

In Lemma 3.3 we described $Q$ as the stream function of Poiseuille flow in $S$, even though it is $\Omega$, rather than $S$, that houses the physical flow field. If we maintain this eccentric but useful point of view, visualizing an actual flow in $S$, it becomes clear that the perturbation stream functions $\psi$ of our weak solutions are in too large a function space: the square of the norm of $V(\Omega)$, which houses these functions $\psi$, is $\int_{S}\left(\nabla^{2} \chi / \hbar\right)^{2}$, whereas that of $V(S)$ is $\int_{S}\left(\nabla^{2} \chi\right)^{2}$. (We have called this latter space $W(S)$ because of $\dot{W}_{2}^{2}(S)$, 
and to avoid confusion with $V(\Omega)$.) For channels of type III (and only for those, it seems) we can prove that the functions $\psi$ are, in fact, in the smaller space $W(S)$, and this will lead to the detailed results, stemming from the notion of 'Poiseuille flow in $S$ ', in section 6.

Although certain estimates in the following proof resemble those in Lemma 3.3, it does not seem possible to by-pass that lemma even for channels of type III; a bound for $\|\psi\|_{V(\Omega)}$ seems necessary in what follows.

THEOREM 3.5. Let $\Omega$ be of type $I I I$, let $\nu>v_{0}$, and let $\Psi=G+\psi$ be the stream function of a weak solution $u$; here $G$ is defined by (3.7), with $\varepsilon=\varepsilon_{0}$ and $\delta=\delta_{0}$ in accord with the choice made after (3.12). Then $\psi$ (more precisely, $\psi \circ F^{\prime}$ ) belongs to the space $W(S)$ defined in Lemma 3.3.

Proof. (i) The property (3.1a) of a weak solution may be written

$$
\nu \int_{S} \frac{1}{h^{2}}\left(\nabla^{2} \chi\right)\left(\nabla^{2} \Psi^{*}\right)+\int_{S} \frac{1}{h^{2}}\left(\chi_{\xi} \Psi_{\eta}-\chi_{\eta} \Psi_{\xi}\right) \nabla^{2} \Psi=0
$$

for all $\chi \in C_{0}^{\infty}(S)$. For the first term, the manipulations in (2.11) and (2.12) are valid even though $\Psi \notin V(\Omega)$; the slightly tortuous calculation yielding the second term is given in Appendix B. Of course, we also obtain (3.13) if we multiply the vorticity equation (2.21c) by $h^{2} \chi$ and integrate over $\mathbb{S}$.

We introduce a fourth mollifier $\tau(., \delta, n) \in C^{\infty}(\mathbf{R} \rightarrow[0,1])$, shown in Fig. $2 ; \tau(\xi, \delta, n)=1$ for $3 / \delta+1 \leqslant|\xi| \leqslant n$, and $\tau(\xi, \delta, n)=0$ for $|\xi| \leqslant 3 / \delta$ and for $|\xi| \geqslant 2 n$. A value $\delta \leqslant \delta_{0}$ will be chosen in due course, but estimates are to be independent of $n$, for $n$ sufficiently large. Define $f_{n}=\tau \psi, \phi_{n}=\tau^{2} \psi, \chi_{n}=h^{2} \phi_{n}$.

Then $\chi_{n} \notin C_{0}^{\infty}(S)$, but it has bounded support, for fixed $n$; we can show that (3.13) holds for $\chi=\chi_{n}$ by a familiar limiting argument, using a sequence $\left\{\psi_{m}\right\}$ in $C_{0}^{\infty}(\Omega)$ such that $\psi_{m} \rightarrow \psi$ in $V(\Omega)$ as $m \rightarrow \infty$; questions of behaviour at infinity in $S$ do not arise, and (even without Lemma 2.2) $h$ and $1 / h$ are bounded on supp $\chi_{n}$ for fixed $n$.

We omit the label $n$ from $f_{n}$ and $\phi_{n}$ henceforth, and use the notation

$$
\begin{aligned}
A(X, Z) & =4 \nu \int_{S}\left\{\left(x X_{\xi}+\lambda X_{\eta}\right)+\left(x^{2}+\lambda^{2}\right) X\right\} \nabla^{2} Z \\
{[X, Y, Z] } & =\int_{S}\left(X_{\xi} Y_{\eta}-X_{\eta} Y_{\xi}\right) \nabla^{2} Z \\
B(X, Y, Z) & =2 \int_{S} X\left(x Y_{\eta}-\lambda Y_{\xi}\right) \nabla^{2} Z
\end{aligned}
$$

Setting $\chi=\chi_{n}$ in (3.13) we then obtain 


$$
\nu \int_{S}\left(\nabla^{2} \phi\right)\left(\nabla^{2} \Psi\right)+A(\phi, \Psi)+[\phi, \Psi, \Psi]+B(\phi, \Psi, \Psi)=0,
$$

where $\Psi=Q+\psi$ because $G=Q$ on supp $\phi$. Note that all terms in $A$ and $B$ contain $\varkappa$ or $\lambda$, which are small on $\operatorname{supp} \phi$. We recall that $Q(\eta)=\frac{3}{4} M\left(\eta-\frac{1}{3} \eta^{3}\right)$; hence

$$
\begin{gathered}
\nu \int_{S}\left(\nabla^{2} \phi\right)\left(\nabla^{2} Q\right)=\nu \int_{S}\left\{\left(\phi_{\xi} Q_{\eta \eta}\right)_{\xi}+\phi Q_{\eta \eta \eta}\right\}=0 \\
{[\phi, Q, Q]=\int_{S}\left(\phi Q_{\eta} Q_{\eta \eta}\right)_{\xi}=0 .}
\end{gathered}
$$

(ii) We wish to bound $\|f\|_{W}^{2}=\int_{S}\left(\nabla^{2} f\right)^{2}$ independently of $n$, and begin by putting (3.14) into a more revealing form; all integrals and norms will be over the set $S_{\delta}=\{\zeta \in S|| \xi \mid>3 / \delta\}$, as they are implicitly in (3.14). A calculation (using $\phi=\tau^{2} \psi, f=\tau \psi$ ) yields

$$
\left\{v\|f\|_{W}^{2}-T_{1}\right\}+A(\phi, \Psi)-\left\{\int Q_{\eta}\left(f_{\eta} f_{\xi \eta}-f_{\xi} f_{\eta \eta}\right)+T_{2}-[\phi, \psi, \psi]\right\}+B(\phi, \Psi, \Psi)=0,
$$

where

$$
\begin{aligned}
& T_{1}=-\nu \int\left\{2 \tau^{\prime 2} \psi \nabla^{2} \psi-\left(2 \tau^{\prime} \psi_{\xi}+\tau^{\prime \prime} \psi\right)^{2}\right\}, \\
& T_{2}=\int \tau \tau^{\prime} Q_{\eta}\left\{\psi_{\xi}^{2}+2 \psi_{\eta}^{2}+\psi\left(2 \psi_{\xi \xi}-\psi_{\eta \eta}\right)\right\}
\end{aligned}
$$

and where the definition of $\nu_{0}$ in Lemma 3.3 shows that

$$
\int Q_{\eta}\left(f_{\eta} f_{\xi \eta}-f_{\xi} f_{\eta \eta}\right) \leqslant v_{0}\|f\|_{W}^{2} .
$$

(iii) We now show that

$$
-A(\phi, \Psi)-B(\phi, \Psi, \Psi) \leqslant \gamma_{1}(\delta)\|f\|_{W}+\gamma_{2}(\delta)\|f\|_{W}^{2}+T_{3}-2 \int \hat{\lambda} \tau \tau^{\prime} \psi^{2} \nabla^{2} \psi
$$

where $\gamma_{j}(\delta) \rightarrow 0$ as $\delta \rightarrow 0(j=1,2)$, and $T_{3}$ is a sum of integrals containing derivatives of $\tau$ (as do $T_{1}$ and $T_{2}$ ) as well as $\varkappa$ or $\lambda$, and is quadratic in $\psi$ (as are $T_{1}$ and $T_{2}$ ). In fact,

$$
T_{3}=\sum \text { const. } \int \mid(x \text { or } \lambda \text { or } \ldots)\left(\tau \tau^{\prime} \text { or } \ldots\right)\left(\nabla^{\alpha} w\right)\left(\nabla^{\beta} \psi\right)|, \quad| \alpha|\leqslant 1, \quad| \beta \mid \leqslant 2,
$$

where 'or ...' refers to terms like $\varkappa^{2}+\lambda^{2}, \tau^{\prime 2}, \tau \tau^{\prime \prime}$.

By Definition 1.1 and a remark following it, $x$ and $\lambda$ are in $L_{\infty}(S)$ for channels of type II, and also in $L_{2}(S)$ for type III; therefore they are in $L_{p}(S)$ for $2 \leqslant p \leqslant \infty$. In addition, $x h$ and $\lambda h$ are now in $L_{q}(S)$ for some $q>2$. Also, $W(S)$ is embedded in $W_{p}^{1}(S)$ for $2 \leqslant p<\infty$, and in $L_{p}(S)$ for $2 \leqslant p \leqslant \infty$. 
We shall use $\gamma(\delta)$ as a generic symbol for functions that tend to zero with $\delta$, and write $t_{3}$ for any integral contributing to $T_{3}$. The first term of $-A(\phi, Q) / 4 v$ is

$$
\begin{gathered}
-\int x \phi_{\xi} Q_{\eta \eta}=-\int x\left(\tau f_{\xi}+\tau^{\prime} f\right) Q_{\eta \eta} \\
\leqslant \text { const. }\|x\|_{L_{2}}\left(\left\|f_{\xi}\right\|_{L_{2}}+\|f\|_{L_{2}}\right) \\
\leqslant \gamma(\delta)\|f\|_{W} .
\end{gathered}
$$

The first term of $-A(\phi, \psi) / 4 v$ is

$$
-\int x \phi_{\xi} \nabla^{2} \psi=-\int x f_{\xi} \nabla^{2} f+t_{3}
$$

where

$$
\begin{gathered}
-\int \varkappa f_{\xi} \nabla^{2} f \leqslant\|x\|_{L_{\infty}}\left\|f_{\xi}\right\|_{L_{2}}\left\|\nabla^{2} f\right\|_{L_{2}} \leqslant \gamma(\delta)\|f\|_{W}^{2}, \\
t_{3}=\int x\left\{\tau \tau^{\prime}\left(2 \psi_{\xi}^{2}-\psi \nabla^{2} \psi\right)+2 \tau^{\prime 2} \psi \psi_{\xi}+\tau \tau^{\prime \prime} \psi \psi_{\xi}+\tau^{\prime} \tau^{\prime \prime} \psi^{2}\right\} .
\end{gathered}
$$

Other terms of $A\left(\phi, \Psi^{p}\right)$ are similar or smaller. Terms in $B\left(\phi, \Psi^{*}, \Psi^{\circ}\right)$ are bounded either as were those of $A(\phi, \Psi)$ or as follows.

$$
-\frac{1}{2} B(\phi, Q, \psi)=-\int \tau f \varkappa Q_{\eta} \nabla^{2} \psi \leqslant \text { const. }\|x h\|_{L_{q}}\|f\|_{L_{r}}\left\|\nabla^{2} \psi / h\right\|_{L_{2}}
$$

where $1 / q+1 / r=\frac{1}{2}$. We now have a choice: either (a) to note that these norms of $x h$ and $\nabla^{2} \psi / h$ both tend to zero as $\delta \rightarrow 0$, and to use a bound $\gamma(\delta, \psi)\|f\|_{W}$, or (b) to note merely that $\|x h\|_{L_{q} \rightarrow 0}$ and $\left\|\nabla^{2} \psi / h\right\|_{L_{z}} \leqslant$ const. by Lemma 3.3 (the constant depending only on the data), and to use a bound $\gamma(\delta)\|f\|_{w}$. In (3.17) we have chosen (b). Next,

where

$$
-\frac{1}{2} B(\phi, \psi, \psi)=-\int f\left(\varkappa f_{\eta}-\lambda f_{\xi}\right) \nabla^{2} \psi-\int \lambda \tau \tau^{\prime} \psi^{2} \nabla^{2} \psi,
$$

$$
\begin{aligned}
-\int f \varkappa f_{\eta} \nabla^{2} \psi & \leqslant\|\varkappa h\|_{L_{q}}\|f\|_{L_{r}}\left\|f_{\eta}\right\|_{L_{s}}\left\|\nabla^{2} \psi / h\right\|_{L_{2}} \\
& \leqslant \gamma(\delta)\|f\|_{W}^{2},
\end{aligned}
$$

provided that $1 / q+1 / r+1 / s=\frac{1}{2}$. A similar estimate holds for the term involving $f \lambda f_{\xi^{*}}$ The $\tau \tau^{\prime}$ term is not absorbed in $T_{3}$ [see (3.17)] because it is cubic in $\psi$.

(iv) Assembling (3.15) to (3.17), we choose $\delta=\delta_{1} \leqslant \delta_{0}$ so small that $\gamma_{2}\left(\delta_{1}\right) \leqslant \frac{1}{2}\left(\nu-\nu_{0}\right)$, and it follows that

$$
\frac{1}{2}\left(\nu-\nu_{0}\right)\|f\|_{W}^{2}-\gamma_{1}\left(\delta_{1}\right)\|f\|_{W} \leqslant \sum_{j=1}^{3} T_{j}-[\phi, \psi, \psi]-2 \int \lambda \tau \tau^{\prime} \psi^{2} \nabla^{2} \psi .
$$

It remains to bound the terms on the right-hand side, all of which contain derivatives of $\tau$; hence they come only from the intervals $3 / \delta_{1}<\xi<3 / \delta_{1}+1$ and $n<\xi<2 n$ for $\xi>0$ (and 
for $\xi<0$ the argument is similar). With $\delta_{1}$ now fixed, $h$ is bounded on the former interval, the contribution of which can therefore be bounded in terms of $\|\psi\|_{\nu(\Omega)}$. In $S_{n}=$ $(n, 2 n) \times(-1,1)$, we have

$$
\left|\tau^{\prime}\left(\xi, \delta_{1}, n\right)\right| \leqslant \text { const. } / n \leqslant \text { const. } / \xi \leqslant \text { const. } / h(\xi, \eta)^{2},
$$

since $h(\xi, \eta) \leqslant$ const. $|\xi|^{\frac{1}{2}}$ for channels of type III. The critical term is

$$
\begin{aligned}
-[\phi, \psi, \psi]_{S_{n}} & =-\int_{S_{n}}\left(\phi_{\xi} \psi_{\eta}-\phi_{\eta} \psi_{\xi}\right) \nabla^{2} \psi=-2 \int_{S_{n}} \tau \tau^{\prime} \psi \psi_{\eta} \nabla^{2} \psi \\
& \leqslant \text { const. }\|f\|_{L_{4}}\left\|\psi_{\eta} / h\right\|_{L_{4}}\left\|\nabla^{2} \psi / h\right\|_{L_{2}} \leqslant \text { const. }\|f\|_{W},
\end{aligned}
$$

where we have used (2.7) for $\psi_{\eta} / h=\hat{v}_{1}$. The last term of (3.18) is of the same type, except for an additional factor $\lambda$. The terms $T$, can be bounded similarly. (In fact, a hypothesis weaker than $h(\xi, \eta) \leqslant$ const. $|\xi|^{1}$ would serve for the $T_{j}$. In $T_{2}$, for example,

$$
\begin{aligned}
\int_{S_{n}} \tau \tau^{\prime} Q_{\eta} \psi_{\xi}^{2} & =\int_{S_{n}} \tau^{\prime} Q_{\eta} f_{\xi} \psi_{\xi}-\int_{S_{n}} \tau^{\prime 2} Q_{\eta} \psi \psi_{\xi} \\
& \leqslant \text { const. }\left\{\|f\|_{W}\left\|\psi_{\xi} / h_{*}\right\|_{L_{2}}+\left\|\psi / h_{*}\right\|_{L_{2}}\left\|\psi_{\xi} / h_{*}\right\|_{L_{\varepsilon}}\right\},
\end{aligned}
$$

where $\left.h_{*}(\xi)=|\xi|.\right)$

Q.E.D.

\section{Regularity of the weak solution}

In this section we examine the regularity of the weak solution $u$, the existence of which was shown in Theorem 3.4. We shall show that $u \in C^{\infty}\left(\bar{U} \rightarrow \mathbf{R}^{2}\right)$ for all bounded domains $U \subset \Omega$, and that there exists an equally smooth pressure $p$ such that $(u, p)$ satisfies (1.1) to (1.3) pointwise. Such results are absolutely standard for weak solutions of the steady Navier-Stokes equations in two and three dimensions (see, for example, [8], [16], [26]), and so we shall only sketch an argument which makes use of the representation $u=\left(\Psi_{y},-\Psi_{x}\right)$.

The proofs of Lemmas 3.2 and 3.3 show that $\Psi^{n}$ has the form $\Psi=G+\psi$, where $G \in C^{\infty}(\bar{U})$ was constructed a priori and $\psi \in V(\Omega)$. The use of this representation in (3.1a) (or direct use of $(2.21 \mathrm{~b})$ ) and various integrations by parts show that $\psi$ satisfies

$$
\begin{aligned}
\nu \int_{\Omega}\left(D^{2} \phi\right)\left(D^{2} \psi\right) & +\int_{\Omega}\left(\psi_{y} \phi_{x}-\psi_{x} \phi_{y}\right) D^{2} \psi+\int_{\Omega}\left(G_{y} \phi_{x}-G_{x} \phi_{y}\right) D^{2} \psi \\
& +\int_{\Omega}\left(\psi_{y} \phi_{x}-\psi_{x} \phi_{y}\right) D^{2} G=-\int_{\Omega} \phi\left\{v D^{2}-\left(G_{y} \frac{\partial}{\partial y}-G_{x} \frac{\partial}{\partial y}\right)\right\} D^{2} G
\end{aligned}
$$


for all $\phi \in C_{0}^{\infty}\left(\Omega \rightarrow \mathbf{R}^{2}\right)$. Eq. (4.1) can be examined with the $L_{p}$ estimates due to Agmon $([2]$, pp. $428-431)$ and the arguments in [5].

THEOREM 4.1. If $\psi \in V(\Omega)$ satisfies (4.1), then $\psi \in C^{\infty}(\bar{U})$ for all bounded domains $U \subset \Omega$.

In fact, one can use [15] to show that $\Psi$ is analytic (i) in $\Omega$, and (ii) at any point $z_{0} \in \partial \Omega$ such that the boundary $\partial \Omega$ is an analytic curve at $z_{0}$.

THWOREM 4.2. If $u$ is a weak solution of (1.1) to (1.4), then $u \in C^{\infty}\left(\bar{U} \rightarrow \mathbf{R}^{2}\right)$ for all bounded domains $U \subset \Omega$. Furthermore, there exists a pressure $p$, with $p \in C^{\infty}(\bar{U} \rightarrow \mathbf{R})$ for all bounded domains $U \subset \Omega$, such that $(u, p)$ satisfies $(1.1)$ to (1.3) pointwise.

Proof. The smoothness of $u$ follows immediately from Theorem 4.1 and the representation $u=\left(\Psi_{y},-\Psi_{x}\right)$. Since $u$ is smooth, we may integrate by parts in (3.1 a):

$$
\int_{\Omega} w \cdot\left\{-v D^{2} u+(u \cdot D) u\right\}=0, \quad \forall w \in J(\Omega)
$$

By application of a standard result ([26], p. 14) to (4.2), there exists a distribution $p \in \mathcal{D}^{\prime}(\Omega)$ such that

$$
-\nu D^{2} u+(u \cdot D) u=-D p
$$

in the sense of distributions. Since $u$ is smooth, the same is true of $p$, and so $(u, p)$ satisfies (1.1) pointwise. The verification that $u$ satisfies (1.2) and (1.3) is standard. Q.E.D.

\section{Pointwise decay at inifinity for channels of type $I^{\prime}$}

\subsection{Channels of type $I^{\prime}$}

In section 3, we proved the existence of a weak solution $u$ to the steady NavierStokes equations in channels $\Omega$ of types I to III, subject to $\nu>v_{0}$ for types II and III. It was shown in section 4 that the velocity $u$ is smooth on bounded subsets of $\Omega$ and that a smooth pressure $p$ exists such that $(u, p)$ satisfies $(1.1)$ to (1.3) pointwise. The final two sections of this paper deal with the behaviour of $u$ and $p$ at infinity in $\Omega$. The present section is concerned with channels of type $I^{\prime}$; such channels are of type $I$ and have the additional properties in Definition 5.1 below. Domains of type I may be regarded, at least roughly, as those for which $h(\zeta)$ grows asymptotically at least like $|\zeta|^{\alpha}, \alpha>\frac{1}{2}$, since $1 / h \in W_{2}^{1}(S)$. In section 6 , we examine $(u, p)$ for channels of type III, and these may be regarded as domains for which $h$ grows no faster than $|\zeta|^{\alpha}$, where $0 \leqslant \alpha \leqslant \frac{1}{2}$. Hence, the results in sections 5 and 6 together cover most cases of $h$ which might arise; exceptions are 
$h(\zeta) \sim|\zeta|^{\frac{1}{4}} \log \log |\zeta|$ and functions $h$ that oscillate very rapidly at infinity. The methods for examining $u$ and $p$ at infinity for channels of type $\mathrm{I}^{\prime}$ and for those of type III are very different; for channels of type $I^{\prime}$, we shall follow a long and tortuous route whose final result is that $u$ tends to zero uniformly at infinity in $\Omega$. This shows that there exists a classical solution $(u, p)$ satisfying (1.1)-(1.4) pointwise for all $\nu>0$ in the case of channels of type I'; however, we are unable to give a rate at which $u$ tends to zero. In section 6 , we examine channels of type III, and Theorem 3.5 will allow us to prove (provided that $v>v_{0}$ ) not only that $u$ tends to zero uniformly whenever the local channel width tends to infinity, but also that the decay occurs at a certain rate. Throughout the rest of this section, we restrict attention to domains of type $I^{\prime}$. The viscosity $v>0$ is arbitrary, but fixed.

Our initial concern is to prove certain results for the stream function $\Psi$ and the corresponding vorticity $\omega \equiv-\nabla^{2} \Psi / h^{2}$. We shall then be in a position to handle the velocity $u$ and the pressure $p$. Certain of the proofs in this section are suggested by those of Gilbarg and Weinberger in [17]; however, the task at hand is different since we shall have to deal with various functions on the lines $\eta= \pm 1$.

In order to derive certain estimates for $\Psi^{*}$ and its derivatives near $\eta= \pm 1$, we need further assumptions on $h$ that exclude highly oscillatory behaviour.

Definition 5.1. A channel $\Omega \subset \mathbf{R}^{2}$ is said to be of type $I^{\prime}$ if it is of type $I$ and the following additional conditions hold. For each $\xi_{0} \in \mathbf{R}$, let $D\left(\xi_{0}\right)=\left(\xi_{0}-1, \xi_{0}+1\right) \times(-1,1)$.

(i) There exist constants $c_{1}, c_{2}>0$ (independent of $\xi_{0}$ ) such that

$$
0<c_{1} \leqslant \frac{h(\xi, \eta)}{h\left(\xi_{0},-1\right)} \leqslant c_{2}, \quad \forall(\xi, \eta) \in D\left(\xi_{0}\right)
$$

$$
\lim _{|\zeta| \rightarrow \infty} \frac{|x(\zeta)|,|\lambda(\zeta)|}{h(\zeta)}=0
$$

where $x=h_{\xi} / h$ and $\lambda=h_{\eta} / h$.

The conditions (5.1) and (5.2) are easily satisfied if $h$ behaves asymptotically as for almost all the channels of type $I$ in Appendix A.

We can combine (5.1) with the property $1 / h \in W_{2}^{1}(S)$ to prove that

or, equivalently,

$$
\lim _{|\zeta| \rightarrow \infty} h(\zeta)=\infty
$$

$$
\lim _{|\xi| \rightarrow \infty} k(\xi)=0, \quad \text { where } k(\xi)=1 / h(\xi,-1) .
$$

Indeed, the assumption (5.I) gives 


$$
\|1 / h\|_{0,2, D(\xi)}^{2} \geqslant 4 c_{2}^{-2} k(\xi)^{2}
$$

and (5.4) then follows immediately since $\|1 / h\|_{0,2, D(\xi)} \rightarrow 0$ as $\xi \rightarrow \infty$.

The velocity $u$ has the form $u=g+v=\left(\Psi_{y},-\Psi_{x}\right)$, where $\Psi^{*}=G+\psi$ and $G_{\text {is }}$ a function only of $\eta$ for large $|\xi|$, while $\psi \in V(\Omega)$. Since $1 / h \in L_{2}(S)$, it follows that

$$
\int_{S}\left(\nabla^{2} G^{2}\right)^{2} / h^{2}<\infty
$$

and if we combine this with the definition of $V(\Omega)$, then we have

$$
\int_{S}\left(\nabla^{2} \Psi\right)^{2} / h^{2}=\int_{S} h^{2} \omega^{2}<\infty
$$

The properties of $G$ and $h$ ensure that

$$
\int_{\mathrm{S}}|\nabla G|^{p} / h^{p}<\infty \text { for all } p \in[2, \infty)
$$

and if we combine this with a similar estimate for $\psi$ (via the representation $v=\left(\psi_{y},-\psi_{x}\right)$ and Lemma 2.1), then we have

$$
\int_{\mathcal{S}}|\nabla \Psi|^{p} / h^{p}<\infty \text { for all } p \in[2, \infty)
$$

Finally, note that (iii) in the proof of Lemma 3.2 gives

$$
\int_{S}|\nabla g|^{2} \leqslant \text { const. }\|1 / h\|_{1,2, s}^{2}<\infty
$$

and since $v \in H(\Omega)$, it follows that

$$
\int_{S}|\nabla u|^{2}=\int_{\Omega}|D u|^{2}<\infty
$$

\subsection{Estimates in a boundary neighbourhood}

Let $k(\xi)=1 / h(\xi,-1)$ and define

$$
\begin{aligned}
& A^{-}=\left\{\zeta \mid-\infty<\xi<\infty,-1<\eta<-1+\min \left(\frac{1}{2}, k(\xi)\right)\right\}, \\
& A^{+}=\left\{\zeta \mid-\infty<\xi<\infty, 1-\min \left(\frac{1}{2}, k(\xi)\right)<\eta<1\right\} .
\end{aligned}
$$

(The domains $A^{-}$and $A^{+}$may be viewed as the images under the map $F^{-1}$ of boundary strips of width order one adjacent to the components of $\partial \Omega$.) If we set $\tilde{\Psi}=\Psi+M / 2$, then $(2.21 \mathrm{c})$ and the fact that $\Psi=G= \pm M / 2$ on $\eta= \pm 1$ give 


$$
\begin{gathered}
\left\{\nu \nabla^{2}-\tilde{\Psi}_{\eta} \frac{\partial}{\partial \xi}+\tilde{\Psi}_{\xi} \frac{\partial}{\partial \eta}\right\} \frac{1}{h^{2}} \nabla^{2} \tilde{\Psi}^{=}=0 \quad \text { in } A^{-}, \\
\tilde{\Psi}=\tilde{\Psi}_{\eta}=0 \quad \text { on } \eta=-1,
\end{gathered}
$$

and similarly in $A^{+}$for $\Psi-M / 2$.

We now prove that

$$
h^{1-|\beta|}\left|\nabla^{\beta} \tilde{\Psi}\right| \in L_{2}\left(A^{-}\right) \text {for }|\beta| \leqslant 2 .
$$

Since Theorem 4.1 shows that $\tilde{\Psi}$ is smooth on compact subsets. of $\bar{\Omega}$, we shall restrict our attention to large values of $|\zeta|$, say, those for which $k(\xi)<\frac{1}{2}$. For each such $\xi_{0}$, set $D_{0}=$ $D\left(\xi_{0}\right) \cap A^{-}=\left(\xi_{0}-1, \xi_{0}+1\right) \times\left(-1,-1+k\left(\xi_{0}\right)\right)$, where $D\left(\xi_{0}\right)$ is given in Definition 5.1. Since $\Psi_{\eta}=0$ on $\eta=1$, it follows that

and so,

$$
\Psi_{\eta}(\zeta)=\int_{-1}^{\eta} \Psi_{\eta \eta}(\xi, t) d t=\int_{-1}^{\eta}\left(\Psi_{\eta \eta}-\lambda \Psi_{\eta}+\varkappa \Psi_{\xi}\right)+\int_{-1}^{\eta}\left(\lambda \Psi_{\eta}-\varkappa \Psi_{\xi}\right), \quad \forall \zeta \in D_{0},
$$

$$
\begin{aligned}
\int_{D_{0}} \Psi_{\eta}^{2} & \leqslant k\left(\xi_{0}\right)^{2}\left\{2 \int_{D_{0}}\left(\Psi_{\eta \eta}-\lambda \Psi_{\eta}+x \Psi_{\xi}\right)^{2}+2 \int_{D_{\mathrm{s}}}\left(\lambda \Psi_{\eta}-\varkappa \Psi_{\xi}\right)^{2}\right\} \\
& \leqslant c_{2}^{2}\left[2 \int_{D_{0}}\left\{\left(\Psi_{\eta \eta}-\lambda \Psi_{\eta}+\varkappa \Psi_{\xi}\right)^{2} / h^{2}\right\}+4 \int_{D_{0}}\left(\lambda \Psi_{\eta} / h\right)^{2}+4 \int_{D_{0}}\left(\varkappa \Psi_{\xi} / h\right)^{2}\right]
\end{aligned}
$$

by (5.1). Similarly,

$$
\int_{D_{0}} \Psi_{\xi}^{2} \leqslant c_{2}^{2}\left[2 \int_{D_{0}}\left\{\left(\Psi_{\xi \eta}-\varkappa \Psi_{\eta}-\lambda \Psi_{\xi}\right)^{2} / h^{2}\right\}+4 \int_{D_{0}}\left(\varkappa \Psi_{\eta} / h\right)^{2}+4 \int_{D_{0}}\left(\lambda \Psi_{\xi} / h\right)^{2}\right]
$$

If we add (5.13a) and (5.13b) and use (2.15) and (5.2), then we have

$$
\int_{D_{0}}|\nabla \Psi|^{2} \leqslant 2 c_{2}^{2} \int_{D_{0}}|\nabla u|^{2}+\frac{1}{2} \int_{D_{0}}|\nabla \Psi|^{2}
$$

for all sufficiently large $\xi_{0}$. Now sum this final inequality over suitable integers $\xi_{0}$, say, $\xi_{0}=M, M+2, \ldots$, to obtain $\nabla \Psi \in L_{2}\left(A^{-}\right)$, since $\nabla u \in L_{2}\left(A^{-}\right)$by $(5.10)$. Now $\tilde{\Psi}=0$ on $\eta=-1$, and so

whence

$$
\begin{aligned}
\left\{h(\zeta) \tilde{\Psi}^{2}(\zeta)\right\}^{2} & \leqslant\left(h(\zeta) \int_{-1}^{-1+k\left(\xi_{0}\right)}\left|\Psi_{\eta}(\xi, t)\right| d t\right)^{2} \\
& \leqslant h(\zeta)^{2} k\left(\xi_{0}\right) \int_{-1}^{-1+k\left(\xi_{0}\right)}\left|\Psi_{\eta}(\xi, t)\right|^{2} d t, \quad \forall \zeta \in D_{0},
\end{aligned}
$$

$$
\int_{D_{0}}(h \tilde{\Psi})^{2} \leqslant c_{2}^{2} \int_{D_{0}}|\nabla \Psi|^{2}
$$


Since $|\nabla \Psi| \in L_{2}\left(A^{-}\right)$, this inequality gives $h \tilde{\Psi} \in L_{2}\left(A^{-}\right)$. For the case $|\beta|=2$ in (5.12), we have

$$
\begin{aligned}
\int_{A^{-}}\left(\Psi_{\eta \eta} / h\right)^{2} & \leqslant 2 \int_{A^{-}}\left\{\left(\Psi_{\eta \eta}-\lambda \Psi_{\eta}+\varkappa \Psi_{\xi}^{\circ}\right) / h\right\}^{2}+2 \int_{A^{-}}\left\{\left(\lambda \Psi_{\eta}-\varkappa \Psi_{\xi}\right) / h\right\}^{2} \\
& \leqslant 2 \int_{A^{-}}|\nabla u|^{2}+\text { const. } \int_{A^{-}}\left|\nabla \Psi^{2}\right|^{2}<\infty
\end{aligned}
$$

since $x / h, \lambda / h \in L_{\infty}(S)$ by (5.2). Similar estimates hold for the other second derivatives of $\Psi$.

With the use of (5.12), equation (5.11) can be examined by the methods in Theorem 3.6 of [5] with the inequalities due to Agmon [2]; after long but routine labours there results

THEоREM 5.2. Let $\tilde{\Psi}=\Psi+M / 2$. Then for each multi-index $\beta, 0 \leqslant|\beta| \leqslant 3$,

(a) $h^{1-|\beta|}|\nabla \beta \tilde{\Psi}| \in L_{2}\left(A^{-}\right)$,

(b) $\left.\left(h^{1 / 2-|\beta|}\left|\nabla \beta \tilde{\Psi^{\prime}}\right|\right)\right|_{\eta=-1} \in L_{2}(\mathbf{R})$,

(c) $h^{-|\beta|} \nabla^{\beta} \tilde{\Psi} \rightarrow 0$ uniformly as $|\zeta| \rightarrow \infty$ in $\overline{A-}$.

Similar results hold in $A^{+}$for $\Psi-M / 2$.

Since $k(\xi) \rightarrow 0$ as $|\xi| \rightarrow \infty$ by (5.4), Theorem 5.2 only provides information about $\Psi$ near $\eta= \pm 1$. For the rest of section 5 , we shall restrict attention to

$$
S_{a}=\{(\xi, \eta) \mid a<\xi<\infty,-1<\eta<1\},
$$

where $a$ is so chosen that $k(\xi)<\frac{1}{2}$ for all $\xi>a$. Similar results will hold for large negative $\xi$. Theorem 5.2 (c) shows that

$$
|\nabla \Psi|=o(h), \quad \omega=o(1) \text { in } \overline{S_{a} \cap A^{+}} \text {and } \overline{S_{a} \cap A^{-}}
$$

and (b) shows that

Note also that

$$
\left.\left(h^{\frac{1}{2}} \omega\right)\right|_{\eta= \pm 1} \in L_{2}(a, \infty)
$$

$$
\omega_{\eta}=\left(-h^{-2} \nabla^{2} \Psi_{\eta}=-h^{-2} \nabla^{2} \Psi_{\eta}-2 \lambda \omega,\right.
$$

and the use of (5.2) and Theorem 5.2(b) together gives

Finally, we have

$$
\left.\left(h^{-\frac{1}{2}} \omega_{\eta}\right)\right|_{\eta= \pm 1} \in L_{2}(a, \infty) \text {. }
$$

$$
\omega_{\xi}=\left(-h^{-2} \nabla^{2} \Psi\right)_{\xi}=-h^{-2} \nabla^{2} \Psi_{\xi}-2 \varkappa \omega,
$$

and the use of (5.2) and Theorem 5.2(c) together gives

$$
\left.\left(h^{-1} \omega_{\xi}\right)\right|_{\eta= \pm 1} \rightarrow 0 \text { as } \xi \rightarrow \infty \text {. }
$$

8-792901 Acta mathematica 144. Imprimé le 13 Juin 1980 


\subsection{Estimates of the vorticity}

The following lemma shows that the velocity $u$ is $o(h)$ in $S_{a}$, and this result will be needed in Theorem 5.4 to prove that $|\nabla \omega| \in L_{2}(S)$.

LEMMA 5.3. $\quad \frac{|u(\zeta)|}{h(\zeta)}=\frac{|\nabla \Psi(\zeta)|}{h(\zeta)^{2}} \rightarrow 0 \quad$ as $|\zeta| \rightarrow \infty \quad$ in $S_{a}$.

Proof. We shall prove the lemma for the case $\eta \in(-1,0]$ since an analogous argument holds for [0, 1). Let $\zeta_{0} \in S_{a}$ be fixed, and note that if $\eta_{0}+1<k\left(\xi_{0}\right)$, then $\zeta_{0} \in A^{-}$, and (5.14) and (5.3) give $\left|\nabla \Psi\left(\zeta_{0}\right)\right| \leqslant$ const. $h\left(\zeta_{0}\right)=o\left(h\left(\zeta_{0}\right)^{2}\right)$. Hence, it suffices to take $\eta_{0} \geqslant-1+k\left(\xi_{0}\right)$. Define the open ball $U \subset S_{a}$ by

$$
U\left(\zeta_{0}\right)=\left\{\zeta|| \zeta-\zeta_{0} \mid<\frac{1}{2} k\left(\xi_{0}\right)\right\}
$$

We wish to use (2.21 c) to examine $\Psi$ in $U$; however, the Agmon estimates are for balls of a fixed size, and so the following affine transformation is needed:

$$
s=\frac{2}{k\left(\xi_{0}\right)}\left(\xi-\xi_{0}\right), \quad t=\frac{2}{k\left(\xi_{0}\right)}\left(\eta-\eta_{0}\right) .
$$

For each $r \in(0,1]$, let $B(r)=\left\{(s, t) \mid s^{2}+t^{2}<r^{2}\right\}$, and note that $B \equiv B(1)$ is the image of $U$ under the map in (5.17).

If we define $f(s, t)=\Psi\left(\xi_{0}+\frac{1}{2} s k\left(\xi_{0}\right), \eta_{0}+\frac{1}{2} t k\left(\xi_{0}\right)\right)$ and $g(s, t)=\omega\left(\xi_{0}+\frac{1}{2} s k\left(\xi_{0}\right), \eta_{0}+\frac{1}{2} t k\left(\xi_{0}\right)\right)$, then $(2.21 \mathrm{c})$ becomes

$$
\nu\left(g_{s s}+g_{t t}\right)-f_{t} g_{s}+f_{s} g_{t}=0 \text { in } B .
$$

The use of (5.18) and an integration by parts give

$$
\nu \int_{B} g\left(\phi_{s s}+\phi_{t i}\right)=-\int_{B} g\left(\phi_{s} f_{t}-\phi_{t} f_{s}\right), \quad \forall \phi \in C_{0}^{\infty}(B \rightarrow \mathbf{R}),
$$

and so, for all $\phi \in C_{0}^{\infty}(B)$,

$$
\left|\int_{B} g\left(\phi_{s s}+\phi_{t t}\right)\right| \leqslant \frac{1}{\nu}\|g\|_{0,2, B}\left\{\left\|\phi_{s}\right\|_{0,4, B}\left\|f_{t}\right\|_{0,4, B}+\left\|\phi_{t}\right\|_{0,4, B}\left\|f_{s}\right\|_{0,4, B}\right\} .
$$

If we apply results of Agmon ([2], pp. 428-429) to this inequality, there results

$$
\|g\|_{1,4 / 3, B(1 / 2)} \leqslant \frac{\text { const. }}{v}\|g\|_{0,2, B}\left\{1+\left\|f_{t}\right\|_{0,4, B}+\left\|f_{s}\right\|_{0,4, B}\right\},
$$

and the constant is independent of $\nu, \zeta_{0}, g$, and $f$. We now estimate the right-hand side of $(5.19)$ : 


$$
\|g\|_{0,2, B}=2 h\left(\xi_{0},-1\right)\|\omega\|_{0,2, U} \leqslant 2 c_{1}^{-1}\|h \omega\|_{0,2, U} \leqslant \text { const., }
$$

where we have used (5.17), (5.1) and (5.7). A similar argument gives

$$
\left\|f_{t}\right\|_{0,4, B}+\left\|f_{s}\right\|_{0,4, B} \leqslant \text { const. } \sqrt{h\left(\xi_{0},-1\right)}\|\nabla \Psi / h\|_{0,4, U}=o\left(h\left(\zeta_{0}\right)\right),
$$

where we have used (5.17), (5.1), (5.9), and (5.3).

The definitions of $f$ and $g$ show that

$$
f_{s s}+f_{t t}=\frac{1}{4} k\left(\xi_{0}\right)^{2} \nabla^{2} \Psi^{2}=-\frac{1}{4} k\left(\xi_{0}\right)^{2} h\left(\zeta_{0}\right)^{2} g,
$$

and a simple argument using (5.1) and (5.2) then gives

$$
\left\|f_{s s}+f_{t t}\right\|_{1,4 / 3, B(1 / 2)} \leqslant \text { const. }\|g\|_{1,4 / 3, B(1 / 2)} .
$$

Combining this final inequality with (5.19) and (5.20), we obtain

$$
\left\|f_{s s}+f_{t t}\right\|_{1,4 / 3, B(1 / 2)}=o\left(h\left(\zeta_{0}\right)\right) .
$$

The Agmon theory now gives

$$
\|f\|_{3,4 / 3, B(1 / 4)} \leqslant \text { const. }\left\{\left\|f_{s s}+f_{t t}\right\|_{1,4 / 3, B(1 / 8)}+\|f\|_{0,2, B(1 / 2)}\right\},
$$

where the constant is independent of $f$. Now $\Psi(\xi,-1)=-M / 2$, and so

$$
|\Psi(\xi, \eta)+M / 2| \leqslant \int_{-1}^{0}\left|\Psi_{\eta}(\xi, t)\right| d t \leqslant c_{2} h(\xi,-1)\left(\int_{-1}^{0} \frac{1}{h^{2}}\left|\Psi_{\eta}(\xi, t)\right|^{2} d t\right)^{1 / 2}=o(h(\zeta))
$$

by (5.1), Lemma 2.1, and the fact that $G_{\eta}$ is bounded. It follows that $\|f\|_{0,2, B(1 / 2)}=o\left(h\left(\zeta_{0}\right)\right)$, and the use of this with (5.21) in (5.22) yields $\|f\|_{3,4 / 3, B(1 / 4)}=o\left(h\left(\zeta_{0}\right)\right)$. The embedding $\left.W_{4 / 3}^{3}\left(B\left(\frac{1}{4}\right)\right) \hookrightarrow C^{1} \overline{\left(B\left(\frac{1}{4}\right)\right.}\right)$ is bounded, and so

$$
\left|\Psi_{\xi}\left(\zeta_{0}\right)\right|=2 h\left(\xi_{0},-1\right)\left|f_{s}(0)\right| \leqslant \text { const. } h\left(\xi_{0},-1\right)\|f\|_{3,4 / 3, B(1 / 4)}=o\left(h\left(\zeta_{0}\right)^{2}\right),
$$

and similarly for $\Psi_{\eta}$

THEOREM 5.4. The vorticity $\omega=-\nabla^{2} \Psi / h^{2}$ is such that $|\nabla \omega| \in L_{2}(S)$.

Proof. It was shown in section 4 that $\Psi \in C^{\infty}(\bar{U})$ for all bounded domains $U \subset S$, and so it suffices to prove that $|\nabla \omega| \in L_{2}\left(S_{a}\right)$. (A similar argument holds for large negative $\xi$.) Recall that $S_{a}=(a, \infty) \times(-1,1)$, with $k(\zeta)<\frac{1}{2}$ there.

Let $\chi \in C^{\infty}(\mathbf{R} \rightarrow[0,1])$ be such that $\chi(r)=0$ for $r<0$ and $\chi(r)=1$ for $r>1$. For each positive integer $n \geqslant a+1$, define $\mu_{n} \in C_{0}^{\infty}(\mathbf{R} \rightarrow[0,1])$ by 


$$
\mu_{n}(\xi)= \begin{cases}\chi(\xi-a), & \xi \leqslant a+1 \\ 1, & a+1 \leqslant \xi \leqslant n \\ 1-\chi(\xi-n), & n \leqslant \xi<\infty\end{cases}
$$

An integration by parts shows that

$$
\begin{aligned}
\nu \int_{S} \mu_{n}|\nabla \omega|^{2}= & -v \int_{S} \mu_{n} \omega \nabla^{2} \omega+\frac{v}{2} \int_{S} \omega^{2} \frac{d^{2} \mu_{n}}{d \xi^{2}} \\
& +\nu \int_{a}^{\infty} d \xi \mu_{n}(\xi)\left\{\omega(\xi, 1) \omega_{\eta}(\xi, 1)-\omega(\xi,-1) \omega_{\eta}(\xi,-1)\right\} .
\end{aligned}
$$

Since $h \omega \in L_{2}(S)$ and $h(\zeta) \rightarrow \infty$ as $|\zeta| \rightarrow \infty$, we have $\omega \in L_{2}(S)$, and so the second term on the right of (5.23) is bounded independently of $n$. Eq. (5.15) shows that the final term is also bounded. Hence

$$
\begin{aligned}
\nu \int_{S} \mu_{n}|\nabla \omega|^{2} & \leqslant \nu\left|\int_{S} \mu_{n} \omega \nabla^{2} \omega\right|+\text { const. } \\
& =\left|\int_{S} \mu_{n} \omega\left(\Psi_{\eta}^{*} \omega_{\xi}-\Psi_{\xi} \omega_{\eta}\right)\right|+\text { const. }
\end{aligned}
$$

by the vorticity equation (2.21 c). Integrating by parts, we have

$$
\begin{aligned}
\nu \int_{S} \mu_{n}|\nabla \omega|^{2} & \leqslant \frac{1}{2}\left|-\int_{S} \omega^{2} \Psi_{\eta} \frac{d \mu_{n}}{d \xi}\right|+\text { const. } \\
& \leqslant \text { const. }\left\{\int_{a<\xi<a+1} \omega^{2}\left|\Psi_{\eta}\right|+\int_{n<\xi<n+1} \omega^{2}\left|\Psi_{\eta}\right|+1\right\} .
\end{aligned}
$$

Now $\omega$ and $\Psi_{\eta}$ are bounded for $\xi \in(a, a+1)$, and Lemma 5.3 and (5.7) give

$$
\int_{n<\xi<n+1} \omega^{2}\left|\Psi_{\eta}\right| \leqslant \text { const. } \int_{n<\xi<n+1} h^{2} \omega^{2} \leqslant \text { const. . }
$$

The theorem then follows.

Q.E.D.

Theorem 5.4, (5.7), and (5.14) now enable us to prove that the vorticity $\omega$ tends to zero pointwise at infinity in $S_{a}$.

CoRolLARY 5.5. $\omega(\xi, \eta) \rightarrow 0$ uniformly as $\xi \rightarrow \infty$.

Proof. Let $\varepsilon>0$ be given. Since $h \omega$ and $|\nabla \omega| \in L_{2}(S)$, we can find a positive integer $N(\varepsilon)$ such that

$$
\int_{n}^{n+1} d \xi \int_{-1}^{1} d \eta\left\{\omega^{2}+\omega_{\eta}^{2}\right\} \leqslant \varepsilon^{2}, \quad \forall n \geqslant N(\varepsilon)
$$


The mean-value theorem ensures the existence of $\tilde{\xi}(n) \in(n, n+1)$ such that

$$
\int_{-1}^{1} d \eta\left\{\omega(\tilde{\xi}, \eta)^{2}+\omega_{\eta}(\tilde{\xi}, \tilde{\eta})^{2}\right\} \leqslant \varepsilon^{2}
$$

and the embedding of $W_{2}^{1}(-1,1)$ in $C[-1,1]$ then gives

$$
|\omega(\tilde{\xi}(n), \eta)| \leqslant \text { const. } \varepsilon, \quad \forall \eta \in[-1,1] .
$$

Furthermore, eq. (5.14) allows us to assume that

$$
|\omega(\xi, 1)|,|\omega(\xi,-1)| \leqslant \varepsilon, \quad \forall \xi \geqslant N(\varepsilon) .
$$

If $A_{n}=\{\tilde{\xi}(n)<\xi<\tilde{\xi}(n+1),-1<\eta<1\}$, then (5.26) and (5.27) together show that $|\omega| \leqslant$ const. $\varepsilon$ on $\partial A_{n}$. From $(2.21 \mathrm{c})$ it follows that $\omega$ and $-\omega$ satisfy a maximum principle in $A_{n}$, and so $|\omega| \leqslant$ const. $\varepsilon$ in $A_{n}$. This is true for all $n \geqslant N(\varepsilon)$, and so $|\omega(\xi, \eta)| \leqslant$ const. $\varepsilon$ for all $\xi \geqslant N(\varepsilon)+1$.

Q.E.D.

\subsection{Averages over arcs of $p$ and $|p|$}

We are now in a position to examine the pressure $p$. Eq. (5.7) gives that $h \omega \in L_{2}\left(S_{\alpha}\right)$, and so

$$
\int_{0}^{1} d \eta \int_{a}^{\infty} d \xi\left\{h(\xi, \eta)^{2} \omega(\xi, \eta)^{2}+h(\xi,-\eta)^{2} \omega(\xi,-\eta)^{2}\right\}=\|h \omega\|_{0,2, S_{a}}^{2}<\infty
$$

The mean-value theorem ensures the existence of a number $\alpha \in(0,1)$ such that

$$
\int_{a}^{\infty} d \xi\left\{h(\xi, \alpha)^{2} \omega(\xi, \alpha)^{2}+h(\xi,-\alpha)^{2} \omega(\xi,-\alpha)^{2}\right\}=\|h \omega\|_{0,2, S_{a}}^{2}
$$

Now, by the form (2.17a) of the Navier-Stokes equations,

$$
\frac{d}{d \xi} \int_{-\alpha}^{\alpha} p(\xi, \eta) d \eta=-\nu\{\omega(\xi, \alpha)-\omega(\xi,-\alpha)\}+\int_{-\alpha}^{\alpha}\left\{u_{1} u_{2 \eta}-u_{2} u_{1 \eta}\right\} d \eta
$$

Since $1 / h \in W_{2}^{1}(S)$, the standard theory of trace yields

$$
\int_{-\infty}^{\infty} \frac{d \xi}{h(\xi, \eta)^{2}} \leqslant \text { const. }\|1 / h\|_{1,2,5}^{2}, \quad \forall \eta \in(-1,1),
$$

and the constant is independent of $\eta$. If we combine this inequality with (5.28), there results 


$$
\begin{aligned}
\int_{a}^{\infty}|\omega(\xi, \alpha)| d \xi & \leqslant\left(\int_{a}^{\infty} h(\xi, \alpha)^{2} \omega(\xi, \alpha)^{2} d \xi\right)^{1 / 2}\left(\int_{a}^{\infty} \frac{d \xi}{h(\xi, \alpha)^{2}}\right)^{1 / 2} \\
& \leqslant \text { const. }\|h \omega\|_{0,2, S_{a}}\|1 / h\|_{1,2, S}
\end{aligned}
$$

and similarly for values at $\eta=-\alpha$. It follows that the first term on the right of (5.29) is an element of $L_{1}(a, \infty)$. Next, by the Schwarz inequality and (2.10),

$$
\left|\int_{-\alpha}^{\infty}\left\{u_{1} u_{2 \eta}-u_{2} u_{1 \eta}\right\} d \eta\right| \leqslant \int_{-1}^{1}\left\{\left|u_{1}\right|\left|u_{2 \eta}\right|+\left|u_{2}\right|\left|u_{1 \eta}\right|\right\} d \eta \leqslant \frac{2}{\pi} \int_{-1}^{1}|\nabla u|^{2} d \eta
$$

and this last term is in $L_{1}(a, \infty)$, because $|\nabla u| \in L_{2}(S)$. It follows that $d\left(\int_{-\alpha}^{\alpha} p(\xi, \eta) d \eta\right) / d \xi$ is in $L_{1}(a, \infty)$. Hence, $\int_{-\alpha}^{\alpha} p(\xi, \eta) d \eta$ has a limit as $\xi \rightarrow \infty$, and, altering $p$ by a constant, we have proved

Lqmia 5.6 There exists a number $\alpha \in(0,1)$ such that

$$
\int_{-\alpha}^{\infty} p(\xi, \eta) d \eta \rightarrow 0 \quad \text { as } \xi \rightarrow \infty
$$

The following result will be needed in the proof of Lemma 5.8.

$$
\text { LEMMA 5.7. } \quad \int_{-1}^{1}|p(\xi, \eta)| d \eta \rightarrow 0 \text { as } \xi \rightarrow \infty \text {. }
$$

Proof. (i) Given any $\varepsilon>0$, choose a positive integer $N=N(\varepsilon)$ such that, for all integers $m \geqslant N$ and all $t \geqslant N$,

$$
\nu^{2} \int_{\xi>m}|\nabla \omega|^{2}, \quad \int_{\xi>m}|\nabla u|^{2}, \quad \int_{-1}^{1}|u(t, \eta)|^{2} d \eta, \quad \int_{-\infty}^{\alpha} p(t, \eta) d \eta \leqslant \varepsilon
$$

these estimates are possible by Theorem 5.4, (5.10), Lemma 2.1, and Lemma 5.6, respectively. Eq. (2.17a) implies that

$$
\int_{m<\xi<m+1}\left|p_{\xi}\right| \leqslant \int_{m<\xi<m+1}\{v|\nabla \omega|+|u||\nabla u|\},
$$

and the use of (5.31) and the Schwarz inequality then gives

$$
\int_{m}^{m+1} d \xi \int_{-1}^{1} d \eta\left|p_{\xi}\right| \leqslant \sqrt{2 \varepsilon}+\sqrt{\varepsilon} \cdot \sqrt{\varepsilon} \leqslant \text { const. } \sqrt{\varepsilon} .
$$

Eq. (5.32) shows that for any $\xi, \xi_{1} \in[m, m+1]$, 


$$
\int_{-1}^{1}|p(\xi, \eta)| d \xi \leqslant \int_{-1}^{1}\left|p\left(\xi_{1}, \eta\right)\right| d \eta+\text { const. } \sqrt{\varepsilon} .
$$

(ii) We now use a weighted form of the Navier-Stokes equations, as in [17], that makes $p_{\eta}$ and $u_{\eta}$ comparable. Let $\mu(\xi)=\max _{\eta \in[-1,1]}\left\{1+|u(\xi, \eta)|^{2}\right\}$; then, by (5.31) and $(2.17 \mathrm{~b})$,

$$
\int_{m}^{m+1} d \xi \int_{-1}^{1}\left\{\frac{1}{\mu(\xi)} p_{\eta}^{2}+\left|u_{\eta}\right|^{2}\right\} d \eta \leqslant 2 v^{2} \int_{\xi>m}|\nabla \omega|^{2}+2 \int_{\xi>m}|\nabla u|^{2}+\int_{\xi>m}|\nabla u|^{2} \leqslant 5 \varepsilon .
$$

If we apply the mean-value theorem to $(5.34)$, we see that there exists $\xi_{1}(m) \in(m, m+1)$ such that

$$
\int_{-1}^{1}\left\{\frac{1}{\mu\left(\xi_{1}\right)} p_{\eta}\left(\xi_{1}, \eta\right)^{2}+\left|u_{\eta}\left(\xi_{1}, \eta\right)\right|^{2}\right\} d \eta \leqslant 5 \varepsilon
$$

The use of (5.31) and (5.35) with the embedding $W_{2}^{1}(-1,1) \hookrightarrow C[-1,1]$ ensures that

$$
\left|u\left(\xi_{1}(m), \eta\right)\right|^{2} \leqslant \text { const. } \varepsilon, \quad \forall \eta \in[-1,1] .
$$

Note that $\mu\left(\xi_{1}\right) \leqslant 1+$ const. $\varepsilon$, and the use of this in (5.35) gives

$$
\int_{-1}^{\xi} p_{\eta}\left(\xi_{1}, \eta\right)^{2} d \eta \leqslant \text { const. } \varepsilon
$$

A standard inequality ([24], p. 117) gives

$$
\int_{-1}^{1} \phi^{2} d \eta \leqslant \text { const. }\left\{\left(\int_{-\infty}^{\alpha} \phi d \eta\right)^{2}+\int_{-1}^{1} \phi_{\eta}^{2} d \eta\right\}, \quad \forall \phi \in W_{2}^{1}(-1,1),
$$

and the constant depends only on the choice of $\alpha \in(0,1)$. If we combine this inequality with $(5.31)$ and $(5.36 a)$, there results

$$
\int_{-1}^{1} p\left(\xi_{1}(m), \eta\right)^{2} d \eta \leqslant \text { const. } \varepsilon .
$$

By $(5.36)$ and the embedding of $W_{2}^{1}(-1,1)$ into $C[-1,1]$,

$$
\left|p\left(\xi_{1}, \eta\right)\right| \leqslant \text { const. } \sqrt{\bar{\varepsilon}}, \quad \forall \eta \in[-1,1] .
$$

Finally, (5.33) and (5.37) together imply that

$$
\int_{-1}^{1}|p(\xi, \eta)| d \eta \leqslant \text { const. } \sqrt{\varepsilon}, \quad \forall \xi \in[m, m+1],
$$

and since the constant is independent of $m$, the proof of the lemma is complete. Q.E.D. 


\subsection{Pointwise decay of the pressure and velocity}

Lemma 5.7 now enables us to prove that the pressure $p$ tends to zero at infinity on the lines $L^{ \pm}=\{a<\xi<\infty, \eta= \pm 1\}$. We shall then use this result in Theorem 5.9 to show that $p(\zeta) \rightarrow 0$ as $|\zeta| \rightarrow \infty$ in $S_{a}$.

\section{LЕМмА 5.8. $p(\xi, \pm 1) \rightarrow 0$ as $\xi \rightarrow \infty$.}

Proof. (i) Let $\varepsilon>0$ be given. It suffices to work with the line $L^{-}$. Let $\zeta_{0}=\left(\xi_{0},-1\right)$ denote an arbitrary fixed point on $L^{-}$. We define polar coordinates $(\varrho, \phi)$, centred at $\zeta_{0}$, by

$$
\boldsymbol{\xi}-\boldsymbol{\xi}_{0}=\varrho \cos \phi, \quad \eta+1=\varrho \sin \phi
$$

where $0 \leqslant \phi \leqslant \pi$. We use $\sim$ to denote a function written in terms of $(\varrho, \phi)$; for example, $\tilde{p}(\varrho, \phi)=p(\xi, \eta)$. Just as (2.16) leads to (2.17), so (2.17) implies that

for $\varrho \in(0,2)$.

$$
\tilde{p}_{\varrho}=-\nu \frac{1}{\varrho} \tilde{\omega}_{\phi}+\frac{1}{\varrho}\left\{\tilde{u}_{1} \tilde{u}_{2 \phi}-\tilde{u}_{2} \tilde{u}_{1 \phi}\right\}
$$

If we integrate (5.38) with respect to $\phi$ from 0 to $\pi$, there results

$$
\frac{d}{d \varrho} \int_{0}^{\pi} \tilde{p}(\varrho, \phi) d p=-\frac{\nu}{\varrho}\{\tilde{\omega}(\varrho, \pi)-\tilde{\omega}(\varrho, 0)\}+\frac{1}{\varrho} \int_{0}^{\pi}\left\{\tilde{u}_{1} \tilde{u}_{2 \phi}-\tilde{u}_{2} \tilde{u}_{1 \phi}\right\} d \phi
$$

We now integrate (5.39) with respect to $\varrho$ from 0 to any $t \in(0,2)$ :

$$
\int_{0}^{\pi} \tilde{p}(t, \phi) d \phi-\pi p\left(\zeta_{0}\right)=-\nu \int_{0}^{t} \frac{d \varrho}{\varrho}\{\tilde{\omega}(\varrho, \pi)-\tilde{\omega}(\varrho, 0)\}+\int_{0}^{t} \frac{d \varrho}{\varrho} \int_{0}^{\pi}\left\{\tilde{u}_{1} \tilde{u}_{2 \phi}-\tilde{u}_{2} \tilde{u}_{1 \phi}\right\} d \phi
$$

The terms on the right of $(5.40)$ are small for all sufficiently large $\xi_{0}$, and we shall prove this in two steps.

(ii) An argument analogous to that for (5.30) yields

$$
\begin{aligned}
& \left|\int_{0}^{t} \frac{d \varrho}{\varrho} \int_{0}^{\pi}\left\{\tilde{u}_{1} \tilde{u}_{2 \phi}-\tilde{u}_{2} \tilde{u}_{1 \phi}\right\} d \phi\right| \\
& \quad \leqslant \text { const. } \int_{0}^{t} \varrho d \varrho \int_{0}^{\pi}|\nabla \tilde{u}|^{2} d \phi \\
& \quad \leqslant \text { const. } \int_{\xi_{0}-2}^{\xi_{0}+2} d \xi \int_{-1}^{1}|\nabla u|^{2} d \eta \rightarrow 0 \text { as } \xi_{0} \rightarrow \infty,
\end{aligned}
$$

since $|\nabla u| \in L_{2}(S)$ by $(5.10)$. 
(iii) Now $\tilde{\omega}(\varrho, \pi)-\tilde{\omega}(\varrho, 0)=\omega\left(\xi_{0}-\varrho,-1\right)-\omega\left(\xi_{0}+\varrho,-1\right)$, and with $h_{0}=h\left(\xi_{0},-1\right)$, we have

$$
\begin{aligned}
\int_{0}^{1 / h_{0}} \frac{d \varrho}{\varrho}|\tilde{\omega}(\varrho, \pi)-\tilde{\omega}(\varrho, 0)| & \leqslant \int_{0}^{1 / h_{0}} \frac{d \varrho}{\varrho}\left|\omega\left(\xi_{0}-\varrho,-1\right)-\omega\left(\xi_{0}+\varrho,-1\right)\right| \\
& \leqslant \frac{2}{h\left(\xi_{0},-1\right)} \sup _{\left|\xi-\xi_{0}\right|<1 / h_{0}}\left|\omega_{\xi}(\xi,-1)\right| \rightarrow 0 \text { as } \xi_{0} \rightarrow \infty .
\end{aligned}
$$

by (5.1) and (5.16). The Schwarz inequality and (5.1) give

$$
\begin{aligned}
& \int_{1 / h_{0}}^{2} \frac{d \varrho}{\varrho}\left\{\left|\omega\left(\xi_{0}-\varrho,-1\right)\right|+\left|\omega\left(\xi_{0}+\varrho,-1\right)\right|\right\} \\
& \quad \leqslant\left[2 h\left(\xi_{0},-1\right) \int_{0}^{2}\left\{\omega\left(\xi_{0}-\varrho,-1\right)^{2}+\omega\left(\xi_{0}+\varrho,-1\right)^{2}\right\} d \varrho\right]^{1 / 2} \\
& \quad \leqslant \text { const. }\left[\int_{0}^{2}\left\{h\left(\xi_{0}-\varrho,-1\right) \omega\left(\xi_{0}-\varrho,-1\right)^{2}+h\left(\xi_{0}+\varrho,-1\right) \omega\left(\xi_{0}+\varrho,-1\right)^{2}\right\} d \varrho\right]^{1 / 2} \\
& \quad \rightarrow 0 \text { as } \xi_{0} \rightarrow \infty
\end{aligned}
$$

by (5.15a).

(iv) The use of (5.41) and (5.42) in (5.40) ensures that

$$
\left|p\left(\zeta_{0}\right)\right| \leqslant \frac{1}{\pi} \int_{0}^{\pi}|\tilde{p}(t, \phi)| d \phi+\varepsilon, \quad \forall t \in(0,2),
$$

when $\xi_{0}$ is sufficiently large. If we multiply (5.43) by $t$ and integrate with respect to $t$ from zero to two, there results

$$
\begin{aligned}
\left|p\left(\zeta_{0}\right)\right| & \leqslant \frac{1}{2 \pi} \int_{0}^{2} t d t \int_{0}^{\pi}|\check{p}(t, \phi)| d \phi+\varepsilon \\
& \leqslant \frac{1}{2 \pi} \int_{\xi_{0}-2}^{\xi_{0}+2} d \xi \int_{-1}^{1}|p(\xi, \eta)| d \eta+\varepsilon \leqslant \text { const. } \varepsilon
\end{aligned}
$$

for all sufficiently large $\xi_{0}$ by Lemma 5.7 .

Q.E.D.

THєовщм 5.9. $p(\xi, \eta) \rightarrow 0$ uniformly as $\xi \rightarrow \infty$.

Proof. (i) Let $\varepsilon>0$ be given. Let $\zeta_{0}=\left(\xi_{0}, \eta_{0}\right) \in S_{a}$ be fixed. We shall prove the theorem for the case $\eta_{0} \in(-1,0]$ since the proof for $\eta_{0} \in[0,1)$ is analogous. We define polar coordinates $(\varrho, \phi)$, centred at $\zeta_{0}$, by

$$
\xi-\xi_{0}=\varrho \cos \phi, \quad \eta-\eta_{0}=\varrho \sin \phi,
$$


where the variable $\phi$ takes values in the interval $[-\pi / 2,3 \pi / 2)$. Consider a circle $\varrho=$ const. $=\varrho_{1}$; if $\varrho_{1} \geqslant 1+\eta_{0}$, then it intersects the wall $\eta=-1$ at

$$
\phi=-\frac{\pi}{2}+\tau\left(\varrho_{1}\right) \equiv \alpha\left(\varrho_{1}\right)
$$

and at

$$
\phi=\frac{3 \pi}{2}-\tau\left(\varrho_{1}\right) \equiv \beta\left(\varrho_{1}\right)
$$

where $\tau(\varrho)=\cos ^{-1}\left\{\left(\eta_{0}+1\right) / \varrho\right\} \in[0, \pi / 2]$; if $\varrho_{1} \leqslant 1+\eta_{0}$, then we define $\tau\left(\varrho_{1}\right)=0, \alpha\left(\varrho_{1}\right)=-\pi / 2$, and $\beta\left(\varrho_{1}\right)=3 \pi / 2$.

(ii) We now integrate (5.38) with respect to $\phi$ from $\alpha(\varrho)$ to $\beta(\varrho)$ :

$$
\int_{\alpha(\varrho)}^{\beta(\varrho)} \tilde{p}_{\varrho} d \phi=-\frac{\nu}{\varrho}\{\tilde{\omega}(\varrho, \beta(\varrho))-\tilde{\omega}(\varrho, \alpha(\varrho))\}+\frac{1}{\varrho} \int_{\alpha(\varrho)}^{\beta(\varrho)}\left\{\left(\tilde{u}_{1}-\overline{\tilde{u}}_{1}\right) \tilde{u}_{2 \phi}-\left(\tilde{u}_{2}-\overline{\tilde{u}}_{2}\right) \tilde{u}_{1 \phi}\right\} d \phi
$$

where

$$
\overline{\tilde{u}}(\varrho)=\{\beta(\varrho)-\alpha(\varrho)\}^{-1} \int_{\alpha(\varrho)}^{\beta(\varrho)} \tilde{u}(\varrho, \phi) d \phi,
$$

and we have used the fact that

$$
\int_{\alpha(\varrho)}^{\beta(\varrho)} \tilde{u}_{\phi}(\varrho, \phi) d \phi=\tilde{u}(\varrho, \beta(\varrho))-\tilde{u}(\varrho, \alpha(\varrho))=0, \quad \forall \varrho \in(0,1)
$$

by continuity if $\alpha(\varrho)=-\pi / 2$ and $\beta(\varrho)=3 \pi / 2$, and otherwise by the fact that $(\varrho, \beta(\varrho))$ and $(\varrho, \alpha(\varrho))$ correspond to points on $\partial S$, where $\tilde{u}$ vanishes. Now

$$
\frac{d}{d \varrho} \int_{\alpha(\varrho)}^{\beta(\varrho)} \tilde{p}(\varrho, \phi) d \phi=\int_{\alpha(\varrho)}^{\beta(\varrho)} \tilde{p}_{\varrho} d \phi+\beta^{\prime}(\varrho) \tilde{p}(\varrho, \beta(\varrho))-\alpha^{\prime}(\varrho) \tilde{p}(\varrho, \alpha(\varrho))
$$

for all $\varrho \in(0,1)$, where ' denotes differentiation. If we integrate (5.45) with respect to $\varrho$ from zero to any $t \in(0,1)$, then the use of (5.44) gives

$$
\begin{aligned}
2 \pi\left|p\left(\zeta_{0}\right)\right| \leqslant\left|\int_{\alpha(t)}^{\beta(t)} \tilde{p}(t, \phi) d \phi\right| & +\left|\int_{0}^{t} d \varrho\left\{\beta^{\prime}(\varrho) \tilde{p}(\varrho, \beta(\varrho))-\alpha^{\prime}(\varrho) \tilde{p}(\varrho, \alpha(\varrho))\right\}\right| \\
& +\nu \int_{0}^{1} \frac{d \varrho}{\varrho}|\tilde{\omega}(\varrho, \beta(\varrho))-\tilde{\omega}(\varrho, \alpha(\varrho))| \\
& +\left|\int_{0}^{t} \frac{d \varrho}{\varrho} \int_{\alpha(\varrho)}^{\beta(\varrho)}\left\{\left(\tilde{u}_{1}-\overline{\tilde{u}}_{1}\right) \tilde{u}_{2 \phi}-\left(\tilde{u}_{2}-\overline{\tilde{u}}_{2}\right) \tilde{u}_{1 \phi}\right\} d \phi\right| .
\end{aligned}
$$

(iii) We proceed to show that the right-hand side of (5.46) tends to zero as $\xi_{0} \rightarrow \infty$; the desired result will then follow easily. The Poincaré inequality ([24], p. 16) gives 


$$
\int_{\alpha(\varrho)}^{\beta(\varrho)}\left\{\tilde{u}_{1}(\varrho, \phi)-\overline{\tilde{u}}_{1}(\varrho)\right\}^{2} d \varrho \leqslant \text { const. } \int_{\alpha(\varrho)}^{\beta(\varrho)} \tilde{u}_{1 \phi}^{2} d \phi
$$

and similarly for $\tilde{u}_{2}$. We can use this inequality to estimate the final term on the right of (5.46) in a manner similar to that for (5.41):

$$
\begin{aligned}
& \left|\int_{0}^{t} \frac{d \varrho}{\varrho} \int_{\alpha(\varrho)}^{\beta(\varrho)}\left\{\left(\tilde{u}_{1}-\overline{\tilde{u}}_{1}\right) \tilde{u}_{2 \phi}-\left(\tilde{u}_{2}-\overline{\tilde{u}}_{2}\right) \tilde{u}_{1 \phi}\right\} d \phi\right| \\
& \quad \leqslant \text { const. } \int_{0}^{t} \varrho d \varrho \int_{\alpha(\varrho)}^{\beta(\varrho)}|\nabla \tilde{u}|^{2} d \phi \\
& \quad \leqslant \text { const. } \int_{\xi_{0}-1}^{\xi_{0}+1} d \xi \int_{-1}^{1}|\nabla u|^{2} d \eta \rightarrow 0 \text { as } \xi_{0} \rightarrow \infty
\end{aligned}
$$

by (5.10). We now estimate the second term on the right of (5.46). The definitions of $\alpha$ and $\beta$ ensure that $\alpha^{\prime}$ and $\beta^{\prime}$ are one-signed, with

$$
\int_{0}^{1}\left|\alpha^{\prime}(\varrho)\right| d \varrho=\int_{0}^{1}\left|\beta^{\prime}(\varrho)\right| d \varrho \leqslant \frac{\pi}{2}
$$

Hence

$$
\left|\int_{0}^{t} d \varrho\left\{\beta^{\prime}(\varrho) \tilde{p}(\varrho, \beta(\varrho))-\alpha^{\prime}(\varrho) \tilde{p}(\varrho, \alpha(\varrho))\right\}\right| \leqslant \pi \max _{\left|\xi-\xi_{0}\right| \leqslant 0}|p(\xi,-1)| \rightarrow 0 \quad \text { as } \xi_{0} \rightarrow \infty
$$

by Lemma 5.8. Finally, we examine the third term on the right of (5.46). Recall that $\alpha(\varrho)=-\pi / 2$ and $\beta(\varrho)=3 \pi / 2$ for $\varrho \leqslant \eta_{0}+1$, so that $\tilde{\omega}(\varrho, \beta(\varrho))=\tilde{\omega}(\varrho, \alpha(\varrho))$ on this interval. Hence the integral may be taken over the interval $\left[\eta_{0}+1,1\right]$, and on this interval, $\tilde{\omega}(\varrho, \beta(\varrho))-\tilde{\omega}(\varrho, \alpha(\varrho))=\omega\left(\xi_{0}-\varrho \sin \tau(\varrho),-1\right)-\omega\left(\xi_{0}+\varrho \sin \tau(\varrho),-1\right)$. Assume for the moment that $\eta_{0}+1 \leqslant 1 / h_{0}$, where $h_{0}=h\left(\zeta_{0}\right)$. The Schwarz inequality and a change of variables give

$$
\begin{gathered}
\int_{1 / h_{0}}^{1}\left|\omega\left(\xi_{0}-\varrho \sin \tau(\varrho),-1\right)\right| \frac{d \varrho}{\varrho} \leqslant\left\{\int \omega^{2} d \varrho\right\}^{1 / 2}\left\{\int \frac{d \varrho}{\varrho^{2}}\right\}^{1 / 2} \\
=\left\{\left(h_{0}-1\right) \int_{\sigma_{0}}^{\sigma_{1}} \omega\left(\xi_{0}-\sigma,-1\right)^{2} \frac{\sigma d \sigma}{\left[\sigma^{2}+\left(\eta_{0}+1\right)^{2}\right]^{1 / 2}}\right\}^{1 / 2},
\end{gathered}
$$

where $\sigma_{0}=\left\{h_{0}^{-2}-\left(\eta_{0}+1\right)^{2}\right\}^{\frac{1}{2}}$ and $\sigma_{1}=\left\{1-\left(\eta_{0}+1\right)^{2}\right\}^{2}$. If we apply (5.1) and (5.15a) to the term above, then

$$
\int_{1 / h_{0}}^{1}\left|\omega\left(\xi_{0}-\varrho \sin \tau(\varrho),-1\right)\right| \frac{d \varrho}{\varrho} \rightarrow 0 \text { as } \xi_{0} \rightarrow \infty
$$

and similarly for the term involving $\omega\left(\xi_{0}+\varrho \sin \tau(\varrho),-1\right)$. We also have 


$$
\begin{aligned}
& \int_{1 / h_{0}}^{1}\left|\omega\left(\xi_{0}-\varrho \sin \tau(\varrho),-1\right)-\omega\left(\xi_{0}+\varrho \sin \tau(\varrho),-1\right)\right| \frac{d \varrho}{\varrho} \\
& \quad \leqslant \frac{2}{h_{0}} \sup _{\left|\xi-\xi_{0}\right|<1 / h_{0}}\left|\omega_{\xi}(\xi,-1)\right| \rightarrow 0 \text { as } \xi_{0} \rightarrow \infty
\end{aligned}
$$

cy $(5.1)$ and (5.16). If we combine (5.49) with the assumption $\eta_{0}+1 \leqslant 1 / h_{0}$, then

$$
\int_{\eta_{0}+1}^{1}\left|\omega\left(\xi_{0}-\varrho \sin \tau(\varrho),-1\right)-\omega\left(\xi_{0}+\varrho \sin \tau(\varrho),-1\right)\right| \frac{d \varrho}{\varrho} \rightarrow 0 \text { as } \xi_{0} \rightarrow \infty .
$$

If $\eta_{0}+\mathrm{I} \geqslant 1 / h_{0}$, then the same result holds by (5.49a). If we combine (5.47), (5.48), and $(5.50)$, then $(5.46)$ yields

$$
\left|p\left(\zeta_{0}\right)\right| \leqslant \frac{1}{2 \pi} \int_{\alpha(t)}^{\beta(t)}|\tilde{p}(t, \phi)| d \phi+\varepsilon, \quad \forall t \in(0,1)
$$

when $\xi_{0}$ is sufficiently large.

(iv) Now multiply the last estimate by $t$ and integrate with respect to $t$ from zero to one, to obtain

$$
\begin{aligned}
\left|p\left(\zeta_{0}\right)\right| & \leqslant \frac{1}{\pi} \int_{0}^{1} t d t \int_{\alpha(t)}^{\beta(t)}|\tilde{p}(t, \phi)| d \phi+\varepsilon \\
& \leqslant \frac{1}{\pi} \int_{\xi_{0}-1}^{\xi_{0}+1} d \xi \int_{-1}^{1}|p(\xi, \eta)| d \eta+\varepsilon \leqslant \text { const. } \varepsilon
\end{aligned}
$$

for a:l sufficiently large $\xi_{0}$ by Lemma 5.7 .

If we define the total head pressure $\Phi$ by $\Phi=p+\frac{1}{2}|u|^{2}$, then a calculation using (1.1) and (1.2) gives

$$
v D^{2} \Phi-\Psi_{y} \Phi_{x}+\Psi_{x} \Phi_{y}=v \omega^{2} \geqslant 0 \text { in } \Omega
$$

where we have used the representation $u=\left(\Psi_{y},-\Psi_{x}\right)$. A change of variables to the strip gives

$$
\nu \nabla^{2} \Phi-\Psi_{\eta} \Phi_{\xi}+\Psi_{\xi} \Phi_{\eta}=\nu h^{2} \omega^{2} \geqslant 0 \text { in } S .
$$

Note that $\Phi$ satisfies a (one-sided) maximum principle. It was shown in the proof of Lemma 5.7 that for any $\varepsilon \in(0,1)$ there exists a positive integer $N=N(\varepsilon)$ as follows: $m \geqslant N$ implies existence of $\xi_{1}(m) \in(m, m+1)$ such that

$$
\left|u\left(\xi_{1}(m), \eta\right)\right|^{2} \leqslant \varepsilon, \quad \forall \eta \in[-1,1] .
$$

Theorem 5.9 allows us to assume that $N$ has been chosen such that

$$
|p(\xi, \eta)| \leqslant \varepsilon, \quad \forall(\xi, \eta) \in(N, \infty) \times[-1,1] .
$$


If $A_{m}=\left(\xi_{1}(m), \xi_{1}(m+1)\right) \times(-1,1)$, then $\Phi \leqslant 3 \varepsilon / 2$ on $\partial A_{m}$ by (5.51), (5.52) and the fact that $u$ vanishes on $\partial S$. The maximum principle ensures that $\Phi(\zeta)=p(\zeta)+\frac{1}{2}|u(\zeta)|^{2} \leqslant 3 \varepsilon / 2$ in $A_{m}$, and (5.51) then implies that $|u(\zeta)|^{2} \leqslant 5 \varepsilon$ in $A_{m}$. Since $m$ may be taken arbitrarily large, we have proved

THEOREM 5.10. $|u(\xi, \eta)| \rightarrow 0$ uniformly as $\xi \rightarrow \infty$.

If we recall that $p$ is determined only up to an additive constant and note that all of our arguments hold equally well for large negative $\xi$, then Theorems $3.4,5.9,5.10$, and the results in section 4 imply

THEOREM 5.11. Let $\Omega \subset \mathbf{R}^{2}$ be a channel of type $\mathrm{I}^{\prime}$. For every $\nu>0$, there exists a classical solution $(u(. ; \nu), p(. ; \nu))$ of $(1.1)$ to $(1.4)$. The velocity u satisfies

(a) $\int_{\Omega}|\nabla u|^{2}<\infty$ and

(b) $u \in C^{\infty}\left(\bar{U} \rightarrow \mathbf{R}^{2}\right)$ for all bounded domains $U \subset \Omega$.

Furthermore, the pressure $p$ satisfies

(c) $p \in C^{\infty}(\bar{U} \rightarrow \mathbf{R})$ for all bounded domains $U \subset \Omega$,

and there exist constants $B_{1}$ and $B_{2}$ such that

$$
\left|p(z)-B_{1}\right|, \quad\left|p(z)-B_{2}\right| \rightarrow 0 \quad \text { as } \quad|z| \rightarrow \infty \text { in } \Omega
$$

upstream and downstream, respectively.

Under additional (reasonable) ssumptions on the asymptotic behaviour of $h$, one can show that all derivatives of $u$ and $p$ tend to zero at infinity. This is the case if $h$ behaves asymptotically as for almost all the examples in Appendix A of channels of type I.

\section{Pointwise decay at infinity for channels of type III}

\subsection{Introductory remarks}

In this section, we examine the behaviour of the velocity $u$ and the pressure $p$ at infinity in channels of type III. The velocity $u$ has the representation $u=\left(\Psi_{y},-\Psi_{x}\right)$ in $\Omega$, where $\Psi$ has the form $\Psi=G+\psi$. The function $G$ was constructed $a$ priori in section 3 to satisfy $G(\zeta)=Q(\eta) \equiv \frac{\mathbf{3}}{4} M\left(\eta-\eta^{3} / 3\right)$ for all sufficiently large $|\xi|$. Hence it suffices to examine the perturbation stream function $\psi$. We shall use Theorem 3.5 and various other estimates to show that suitable derivatives of $\psi$ tend to zero uniformly at infinity in $S$. We shall also prove that the pressure $p(\zeta)$ tends to $\mp \infty$ uniformly as $\xi \rightarrow \pm \infty$, and an 
estimate for the rate of growth will be given. Finally, if $\chi(\zeta)$ and $\lambda(\zeta)$ go to zero like $|\zeta|^{-\gamma}$ in $S$, for some $\gamma>0$, then the same decay rate will be shown to hold for various derivatives of $\psi$. All of our results for $\psi$ in $S$ may be translated into estimates for $\psi$ in $\Omega$ by the relation $\left|D^{\beta} \psi\right|=\left|\nabla^{\beta} \psi\right| / h^{|\beta|},|\beta| \leqslant 1$; it is important to note that $1 / h$ is bounded above on $S$ since (2.22) shows that $\inf h(\zeta)>0$ for channels of type II and III. Throughout this section, the viscosity $v$ will be fixed and will satisfy $v>v_{0}$ (cf. Theorem 3.4). For convenience, we now list those properties of domains of type III which will be used in this section:

$$
\begin{gathered}
\varkappa(\zeta) \lambda(\zeta) \rightarrow 0 \quad \text { uniformly as } \quad|\xi| \rightarrow \infty ; \\
x, \lambda \in L_{2}(S) ; \\
h(\zeta) \leqslant \text { const. }(1+|\zeta|)^{\frac{1}{2}}, \quad \forall \zeta \in S .
\end{gathered}
$$

It is property (6.3) that prevents us from applying the methods of section 5 to the Navier-Stokes equations in a channel of type III; indeed, (6.3) gives $\int_{S}(1 / h)^{2}=\infty$, and then $h \omega \notin L_{2}(S)$, because $Q_{\eta \eta} / h \notin L_{2}(S)$ while $\nabla^{2} \psi / h \in L_{2}(S)$. Similarly,

$$
\int_{\Omega}|D u|^{2}=\int_{S}|\nabla u|^{2}=\infty
$$

In section 5, we used the fact that $h \omega,|\nabla u| \in L_{2}(S)$, and other estimates, to prove that the pressure $p$ has finite limits at infinity in $S$. This result was then used with a maximum principle for the total head pressure $\Phi=p+\frac{1}{2}|u|^{2}$ to prove that $u$ goes to zero uniformly at infinity. However, for the present problem, the condition (6.3) will be used in Theorem 6.3 to show that $p$ is unbounded at infinity, and so the maximum principle for $\Phi$ cannot be used to estimate $u$. Although we know that $h \omega=-\left(\nabla^{2} G+\nabla^{2} \psi\right) / h \notin L_{2}(S)$, Theorem 3.5 shows that the perturbation stream function $\psi$ satisfies the stronger estimate

$$
\int_{S}\left(\nabla^{2} \psi\right)^{2}=\int_{S}\left(\psi_{\xi \xi}^{2}+2 \psi_{\xi \eta}^{2}+\psi_{\eta \eta}^{2}\right)<\infty
$$

and it is this estimate which will be used throughout this section. Since $\psi=\psi_{\eta}=0$ on $\partial S$, the inequality (2.10) shows that

$$
\int_{S}\left(\psi^{2}+|\nabla \psi|^{2}\right) \leqslant \text { const. } \int_{S}\left(\psi_{\xi \xi}^{2}+2 \psi_{\xi \eta}^{2}+\psi_{\eta \eta}^{2}\right)<\infty,
$$

and so $\psi \in \stackrel{\circ}{W}_{2}^{2}(S)$. 


\subsection{Estimates from the vorticity equation}

It will suffice to work in the domain

$$
S_{\tilde{a}}=\{(\xi, \eta) \mid \tilde{a}<\xi<\infty,-1<\eta<1\},
$$

where $\tilde{a}>0$ has been so chosen that

$$
G(\xi, \eta)=Q(\eta) \equiv \frac{3}{4} M\left(\eta-\eta^{3} / 3\right) \text { for } \xi>\tilde{a} .
$$

Similar results will always hold for large negative $\xi$. If we use the representation $\Psi=G+\psi$ in (2.21c) and recall that $h \varkappa=h_{\xi}$ and $h \lambda=h_{\eta}$, then we find that $\psi$ satisfies the equation

$$
\begin{aligned}
L \psi & \equiv \nu \nabla^{4} \psi-Q_{\eta} \nabla^{2} \psi_{\xi}+Q_{\eta \eta \eta} \psi_{\xi} \\
= & \left\{\psi_{\eta}\left(\frac{\partial}{\partial \xi}-2 \varkappa\right)-\psi_{\xi}\left(\frac{\partial}{\partial \eta}-2 \lambda\right)\right\} \nabla^{2} \psi+B(\psi)+F \text { in } S_{\tilde{a}} \\
& \psi=\psi_{\eta}=0 \text { on } \eta= \pm 1
\end{aligned}
$$

where $\nabla^{4}=\left(\nabla^{2}\right)^{2}$ denotes the biharmonic operator, and

$$
B(\psi)=4 \nu\left\{\left(\varkappa \frac{\partial}{\partial \xi}+\lambda \frac{\partial}{\partial \eta}\right)-\left(\varkappa^{2}+\lambda^{2}\right)\right\} \nabla^{2} \psi-2 \varkappa Q_{\eta} \nabla^{2} \psi-2 Q_{\eta \eta}\left(\varkappa \psi_{\eta}-\lambda \psi_{\xi}\right),
$$

and

$$
F=4 v\left\{\lambda \frac{\partial}{\partial \eta}-\left(\varkappa^{2}+\lambda^{2}\right)\right\} Q_{\eta \eta}-2 \varkappa Q_{\eta} Q_{\eta \eta}
$$

Let $J$ be any fixed positive integer greater than $\tilde{a}$. For each integer $j \geqslant J$, set

and

$$
S_{j}=\{j<\xi<j+1,-1<\eta<1\}
$$

$$
S_{j}^{1}=\{j-1<\xi<j+2,-1<\eta<1\}
$$

Assume that $j \geqslant J+1$, multiply (6.6a) by any $\phi \in C_{0}^{\infty}\left(S_{j}^{1} \rightarrow \mathbf{R}\right)$, so that integration only over $S_{j}^{1}$ is implied in what follows, and integrate by parts:

$$
\begin{aligned}
\nu\left|\int \psi \nabla^{4} \phi\right| \leqslant & \left|\int\left(Q_{\eta} \phi_{\xi} \nabla^{2} \psi+Q_{\eta \eta} \phi \psi_{\xi}\right)\right| \\
& +\left|\int\left(\phi_{\xi} \psi_{\eta}-\phi_{\eta} \psi_{\xi}+2 x \phi \psi_{\eta}-2 \lambda \phi \psi_{\xi}\right) \nabla^{2} \psi\right| \\
& +\left|\int \phi B(\psi)\right|+\left|\int \phi F\right| .
\end{aligned}
$$


We now estimate the terms on the right of (6.7). Up to the statement of Theorem 6.2, the norms of $W_{p}^{m}\left(S_{j}^{1}\right)$ and $W_{p}^{m}\left(S_{j}\right)$ will be denoted by $\|\cdot\|_{m, p}$ and $\|\cdot\|_{m, p . S_{j}}$, respectively. Since $x, \lambda \in L_{2}(S) \cap L_{\infty}(S)$ by (6.1) and (6.2), it follows that

$$
\left|\int \phi F\right| \leqslant \text { const. }\|\phi\|_{0.2}\left(\|x\|_{0,2}+\|\lambda\|_{0,2}\right)
$$

and the constant is independent of $j$ and $\phi$. We now estimate the integral involving $B(\psi)$. First, recall that $\varkappa_{\xi}+\lambda_{\eta}=0$, so that

$$
\left|\int \phi\left(x \nabla^{2} \psi_{\xi}+\lambda \nabla^{2} \psi_{\eta}\right)\right|=\left|\int \nabla^{2} \psi\left(x \phi_{\xi}+\lambda \phi_{\eta}\right)\right| \leqslant \text { const. }\|\psi\|_{2,2}\|\phi\|_{1,2}
$$

If we combine this estimate with ones for the other terms in $B(\psi)$, then it follows that

$$
\left|\int \phi B(\psi)\right| \leqslant \text { const. }\|\psi\|_{2,2}\|\phi\|_{1,2}
$$

and the constant is independent of $\phi$ and $j$. For the other terms in (6.7), we have

and

$$
\left|\int\left(Q_{\eta} \phi_{\xi} \nabla^{2} \psi+Q_{\eta \eta \eta} \phi \psi_{\xi}\right)\right| \leqslant \text { const. }\|\psi\|_{2,2}\|\phi\|_{1,2}
$$

$$
\left|\int\left(\phi_{\xi} \psi_{\eta}-\phi_{\eta} \psi_{\xi}+2 \varkappa \phi \psi_{\eta}-2 \lambda \phi \psi_{\xi}\right) \nabla^{2} \psi\right| \leqslant \text { const. }\|\psi\|_{2,2}\|\psi\|_{1, t}\|\phi\|_{1, s}
$$

where $1 / s+1 / t=\frac{1}{2}$. Standard theory shows that the embedding $W_{2}^{2}\left(S_{j}^{1}\right) \hookrightarrow W_{t}^{1}\left(S_{j}^{1}\right)$ is bounded for all $t \geqslant 1$, and so

$$
\left|\int\left(\phi_{\xi} \psi_{\eta}-\phi_{\eta} \psi_{\xi}+2 x \phi \psi_{\eta}-2 \lambda \phi \psi_{\xi}\right) \nabla^{2} \psi\right| \leqslant \text { const. }\|\psi\|_{2,2}^{2}\|\phi\|_{1, s}, \quad \forall s>2,
$$

and the constant depends only on $s$. If we use the estimates $(6.8)$ to (6.11) in (6.7), then

$$
\left|\int \psi \nabla^{4} \phi\right| \leqslant c(j ; s)\|\phi\|_{1, s}, \quad \forall \phi \in C_{0}^{\infty}\left(S_{j}^{1} \rightarrow \mathbf{R}\right),
$$

and for all $s>2$. Since $\psi \in \stackrel{\circ}{W}_{2}^{2}(S)$ and $\varkappa, \lambda \in L_{2}(S)$, the constants $c(j: s)$ satisfy

$$
\sum_{j=J+1}^{\infty} c(j ; s)^{2}<\infty
$$

Applying to (6.12) the arguments in [2], pp. 428-431, we find that 


$$
\|\psi\|_{3, s^{\prime}, s_{j}} \leqslant \text { const. }\left(c(j ; s)+\|\psi\|_{2,2}\right)
$$

where $1 / s+1 / s^{\prime}=1$ and the constant depends on $s^{\prime}$, but not on $j$. Since $s>2$ is arbitrary, it follows that $s^{\prime} \in(1,2)$. The embedding $W_{s^{\prime}}^{3}\left(S_{j}\right) \hookrightarrow C_{1}\left(\bar{S}_{f}\right)$ is bounded, and so (6.13) and (6.14) give the following lemma (also stated for large negative $\xi$ ).

LEMMA 6.1. Let $\Omega$ be a channel of type $I I I$ and let $\psi$ denote the perturbation stream function. Then

$$
\psi(\xi, \eta),|\nabla \psi(\xi, \eta)| \rightarrow 0 \quad \text { uniformly as } \quad|\xi| \rightarrow \infty .
$$

If we combine this lemma with the result from section 4 that $\psi \in C^{\infty}(\bar{U})$ for all bounded domains $U \subset S$, we see that $|\nabla \psi|$ is bounded on $\bar{S}$. This enables us to improve (6.11):

$$
\left|\int\left(\phi_{\xi} \psi_{\eta}-\phi_{\eta} \psi_{\xi}+2 \varkappa \phi \psi_{\eta}-2 \lambda \phi \psi_{\xi}\right) \nabla^{2} \psi\right| \leqslant \text { const. }\|\psi\|_{2,2}\|\phi\|_{1,2},
$$

and the constant is independent of $\phi$ and $j$. Use of this inequality with (6.8) to (6.10) in (6.7) yields

$$
\left|\int \psi \nabla^{4} \phi\right| \leqslant d(j)\|\phi\|_{1,2}, \quad \forall \phi \in C_{0}^{\infty}\left(S_{j}^{1} \rightarrow \mathbf{R}\right),
$$

where the constants $d(j)$ satisfy

Standard theory gives

$$
\sum_{j=J+1}^{\infty} d(j)^{2}<\infty
$$

and so it follows that

$$
\|\psi\|_{3,2, S_{j}} \leqslant \text { const. }\left(d(j)+\|\psi\|_{2,2}\right),
$$

$$
\sum_{J+1}^{\infty}\|\psi\|_{3,2, S_{j}}^{2} \leqslant \text { const. }\left(\sum_{J+1}^{\infty} d(j)^{2}+\sum_{J+1}^{\infty}\|\psi\|_{2,2}^{2}\right)<\infty
$$

since $\psi \in \stackrel{\mathscr{W}}{2}_{2}^{2}(S)$. A similar argument holds for large negative $\xi$, and so we have shown that $\psi \in W_{2}^{3}(S)$. By use of this fact in (6.6a), it follows that $\nabla^{4} \psi \in L_{2}(S)$, and standard theory then gives $\psi \in W_{2}^{4}(S)$. The embedding $W_{2}^{4}(S) \hookrightarrow W_{s}^{3}(S)$ is bounded for all $s \geqslant 2$, and so (6.6a) ensures that $\nabla^{4} \psi \in L_{s}(S)$ for all $s \geqslant 2$. It follows that $\psi \in W_{s}^{4}(S)$ for all $s \geqslant 2$. The embedding $W_{s}^{4}(S) \hookrightarrow C^{3}(\bar{S})$ is bounded for all $s>2$, and so for each multi-index $\alpha$ with $|\alpha| \leqslant 3$, we have $\nabla^{\alpha} \psi(\xi, \eta) \rightarrow 0$ uniformly as $|\xi| \rightarrow \infty$. We summarize our results in the following theorem.

THEOREM 6.2. The perturbation stream function $\psi$ is an element of $W_{s}^{4}(S)$ for all $s \geqslant 2$, and for each multi-index $\alpha, 0 \leqslant|\alpha| \leqslant 3$,

$$
\nabla^{\alpha} \psi(\xi, \eta) \rightarrow 0 \quad \text { uniformly as } \quad|\xi| \rightarrow \infty .
$$


If the behaviour of suitable derivatives of $\varkappa$ and $\lambda$ is known, then results for higher derivatives of $\psi$ can be gained; for example, if all derivatives of $\varkappa$ and $\lambda$ are in $L_{2}(S)$, then all derivatives of $\psi$ are in $L_{2}(S)$ and tend to sero at infinity.

The velocity $u$ has the representation $u=g+v$ in $\Omega$, where $g=\left(G_{y},-G_{x}\right)$ and $v=\left(\psi_{y},-\psi_{x}\right)$. Recall that $G(\zeta)$ is constructed explicitly in section 3, and so $g$ is known explicitly when the conformal mapping between $\Omega$ and $S$ is known; however, since we are interested in the behaviour at infinity, it suffices to know the asymptotic form of the mapping function. Now

$$
|v|=|D \psi|=|\nabla \psi| / h=o(\mathbf{l} / h)
$$

by Theorem 6.2 with $|\alpha|=1$, while

$$
|g|=|\nabla G| / h=\frac{3}{4} M\left(1-\eta^{2}\right) / h
$$

sufficiently for upstream and downstream. If $h(\zeta) \rightarrow \infty$ as $|\zeta| \rightarrow \infty$ in $S$, then clearly $|u| \rightarrow 0$ like $1 / h$. If $h(\zeta)$ goes to a finite (non-zero) limit, then $g$ behaves like a Poiseuille velocity field. In either case, $v$ goes to zero uniformly at infinity.

We now examine the pressure $p$ at infinity in $S$. Recall that (6.3) gives $\int_{S} 1 / h^{2}=\infty$.

THEOREM 6.3. The pressure $p$ satisfies

$$
p_{\eta}(\zeta), p_{\xi}(\zeta)+3 M v / 2 h(\zeta)^{2}=o\left(1 / h(\zeta)^{2}\right) \quad \text { as } \quad|\zeta| \rightarrow \infty \text { in } S .
$$

Furthermore, $p(\xi, \eta) \rightarrow \mp \infty$ uniformly as $\xi \rightarrow \pm \infty$ and

$$
-p\left(\zeta_{0}\right) / \int_{0}^{\xi_{0}} d \xi \int_{-1}^{1} \frac{1}{h^{2}} d \eta \rightarrow \frac{3}{4} M v \text { uniformly as }\left|\xi_{0}\right| \rightarrow \infty .
$$

Proof. Eq. (2.18) gives

$$
\left.\begin{array}{l}
\nu \omega_{\eta}-\hat{u}_{1}\left(\hat{u}_{2 \eta}+\varkappa \hat{u}_{1}\right)+\hat{u}_{2}\left(\hat{u}_{1 \eta}-x \hat{u}_{2}\right)=-p_{\xi} \\
-\nu \omega_{\xi}+\hat{u}_{1}\left(\hat{u}_{2 \xi}-\lambda \hat{u}_{1}\right)-\hat{u}_{2}\left(\hat{u}_{1 \xi}+\lambda \hat{u}_{2}\right)=-p_{\eta}
\end{array}\right\} \text { in } S
$$

where $\omega=-\nabla^{2} \Psi / h^{2}, \hat{u}_{1}=\Psi_{\eta} / h$ and $\hat{u}_{2}=-\Psi_{\xi} / h$. Let $N$ be a large positive integer such that $G(\zeta)=\frac{3}{4} M\left(\eta-\eta^{3} / 3\right)$ in $A \equiv S \cap\{|\xi|>N\}$. At points in $A$, we have

$$
\begin{aligned}
v \omega_{\eta} & =v \frac{\partial}{\partial \eta}\left\{-\left(\nabla^{2} \psi+G_{\eta \eta}\right) / h^{2}\right\}=-v\left(\nabla^{2} \psi_{\eta}-\frac{3}{2} M\right) / h^{2}+2 \lambda v\left(\nabla^{2} \psi+G_{\eta \eta}\right) / h^{2} \\
& =3 M v / 2 h^{2}+o\left(1 / h^{2}\right)
\end{aligned}
$$

by (6.1) and Theorem 6.2. Analogous estimates for the other terms in (6.16) give the first result of the theorem. 
Eq. (2.22) shows that $1 / h$ is bounded on $\bar{S}$, and so

We have

$$
|\nabla p| \leqslant \text { const. in } \bar{S} \text {. }
$$

$$
\begin{aligned}
p\left(\xi_{0}, \eta\right) & =p(0, \eta)+\int_{0}^{\xi_{0}} \frac{\partial p}{\partial \xi}(\xi, \eta) d \xi \\
& =p(0, \eta)-\int_{0}^{\xi_{0}}\left\{3 M \nu / 2 h^{2}+o\left(1 / h^{2}\right)\right\} d \xi
\end{aligned}
$$

and so the mean value

$$
\bar{p}\left(\xi_{0}\right) \equiv \frac{1}{2} \int_{-1}^{1} p\left(\xi_{0}, \eta\right) d \eta=\frac{1}{2} \int_{-1}^{1} p(0, \eta) d \eta-\int_{0}^{\xi_{0}} d \xi \int_{-1}^{1}\left\{3 M \nu / 4 h^{2}+o\left(1 / h^{2}\right)\right\} d \eta .
$$

It follows that

$\mathrm{Eq} .(6.17)$ gives

$$
\lim _{\left|\xi_{0}\right| \rightarrow \infty}-\bar{p}\left(\xi_{0}\right) /\left(\int_{0}^{\xi_{0}} d \xi \int_{-1}^{1} \frac{1}{h^{2}} d \eta\right)=\frac{3}{4} M \nu
$$

$$
\left|p\left(\xi_{0}, \eta_{0}\right)-\bar{p}\left(\xi_{0}\right)\right| \leqslant \int_{-1}^{1}\left|p_{\eta}\left(\xi_{0}, \eta\right)\right| d \eta \leqslant \text { const., } \quad \forall \eta_{0} \in[-1,1],
$$

and the use of this with (6.18) immediately gives (6.15).

Q.E.D.

\subsection{Decay rates}

We now examine the asymptotic behaviour of $\psi(\zeta)$ as $\xi \rightarrow \infty$ when $\varkappa(\zeta)$ and $\lambda(\zeta)$ are known to decay like some power of $\xi$ as $\xi \rightarrow \infty$; more precisely, we shall assume for the rest of this section that there exist constants $\tilde{C}, \gamma>0$ such that

$$
|x(\zeta)|,|\lambda(\zeta)| \leqslant \tilde{C} \xi^{-\gamma}, \quad \forall(\xi, \eta) \in S_{\tilde{a}}
$$

where $S_{t}=\{t<\xi<\infty,-1<\eta<1\}, t>0$, and $\tilde{a} \geqslant 3$ has been chosen so large that $G=Q$ in $S_{\tilde{a}}$. We shall prove that (6.19) implies that suitable derivatives of $\psi$ grow no faster than $\xi^{-\gamma}$ in $S_{\tilde{a}}$. (For the channels of type III, with nonoscillatory boundaries, in Appendix A, $\gamma \geqslant 1$.) Similar results hold for large negative $\xi$ when an analogous version of (6.19) is true.

For each fixed $a \geqslant \tilde{a}$ and for $n=1$ or 2 , define the Banach spaces $C_{n}$ and $E_{n}$ by

Eq. (6.6) gives

$$
\begin{gathered}
C_{n}=C_{n}\left(\bar{S}_{a}\right)=\left\{f \in C\left(\bar{S}_{a} \rightarrow \mathbf{R}^{n}\right)\left|\|f\|_{c_{n}} \equiv \sup _{\zeta \in S_{a}}\right| f(\zeta) \mid<\infty\right\}, \\
E_{n}=E_{n}\left(S_{a}\right)=\left\{f \in C\left(\bar{S}_{a} \rightarrow \mathbf{R}^{n}\right)\left|\|f\|_{E_{n}} \equiv \sup _{\zeta \in S_{a}}\right| \xi^{\gamma} f(\zeta) \mid<\infty\right\} .
\end{gathered}
$$

$$
L \psi \equiv \nu \nabla^{4} \psi-Q_{\eta} \nabla^{2} \psi_{\xi}+Q_{\eta \eta \eta} \psi_{\xi}=\psi_{\eta} \nabla^{2} \psi_{\xi}-\psi_{\xi} \nabla^{2} \psi_{\eta}+\tilde{F} \quad \text { in } S_{\tilde{a}}
$$


where

$$
\psi=\psi_{\eta}=0 \text { on } \eta= \pm 1,
$$

$$
\widetilde{F}=B(\psi)+F-2 x \psi_{\eta} \nabla^{2} \psi+2 \lambda \psi_{\xi} \nabla^{2} \psi,
$$

and the definitions of $B(\psi)$ and $F$ appear immediately after (6.6). Theorem 6.2 ensures that the first three derivatives of $\psi$ are bounded on $\bar{S}$, and inspection of the definitions of $B(\psi)$ and $F$ shows that

for each $a \geqslant \tilde{a}$.

$$
\widetilde{F} \in E_{1}\left(S_{a}\right)
$$

In order to examine (6.20), we shall use the Green function for the adjoint operator $L^{*}$, where

$$
L^{*} \phi=\nu \nabla^{4} \phi+\nabla^{2}\left(Q_{\eta} \phi_{\xi}\right)-Q_{\eta \eta \eta} \phi_{\xi} .
$$

It was shown in section 4 of [5] that if $v>v_{0}$ (which is the present case), then for each fixed $\zeta_{0} \in S$, there exists a Green function $K\left(\zeta_{0},.\right) \in \stackrel{\circ}{W}_{2}^{2}(S)$ such that

$$
\left.\begin{array}{l}
L^{*} K\left(\zeta_{0}, \zeta\right)=\delta\left(\zeta-\zeta_{0}\right) \text { in } S \\
K\left(\zeta_{0}, \zeta\right)=K_{\eta}\left(\zeta_{0}, \zeta\right)=0 \text { on } \eta= \pm 1
\end{array}\right\}
$$

where $\delta$ denotes the Dirac distribution. The following lemma is Theorem 4.1 of [5].

LEMMA 6.4. Let $S=\mathbf{R} \times(-1,1)$ and assume that $\boldsymbol{\nu}>v_{0}$. Then for each $\zeta_{0} \in S$,

(a) there exists a Green function $K\left(\zeta_{0},.\right) \in W_{2}^{2}(S)$ which satisfies (6.22);

(b) there exist positive constants $C=C(v), b=b(v)$ and $c=c(v)$ independent of $\zeta_{0}$, such that, with $r=\left|\zeta-\zeta_{0}\right|$,

$$
\left|\nabla_{0}^{\alpha} \nabla^{\beta} K\left(\xi_{0}, \zeta\right)\right| \leqslant\left\{\begin{array}{l}
C / r^{2} \text { for }\left|\xi-\xi_{0}\right| \leqslant 2, \\
C \exp \left\{-b\left(\xi_{0}-\xi\right)\right\} \text { for } \xi-\xi_{0} \leqslant-2, \\
C \exp \left\{-c\left(\xi-\xi_{0}\right)\right\} \text { for } \xi-\xi_{0} \geqslant 2,
\end{array}\right.
$$

where $\alpha$ and $\beta$ are any multi-indices satisfying $|\alpha|+|\beta|=4$.

(c) Analogous bounds hold for derivatives of $K$ of order $3,2,1$ or 0 with $C / r^{2}$ replaced by $C / r, C(1+|\log r|), C$ and $C$, respectively, for $\left|\xi_{0}-\xi\right| \leqslant 2$.

For each $a \geqslant \tilde{a}+1$, let $\mu=\mu(. ; a) \in C^{\infty}(\mathbf{R} \rightarrow[0,1])$ be a mollifier such that $\mu(t ; a)=1$ for $t \geqslant a$ and $\mu(t ; a)=0$ for $t \leqslant a-1$. If we multiply $(6.20)$ by $\mu(\xi ; a) K\left(\zeta_{0}, \zeta\right)$, integrate over $S_{\tilde{a}}$, and apply Lemma 6.4, then we have for $\xi_{0}>a$

$$
\int_{a-1<\xi} \mu K\left(\zeta_{0}, \zeta\right)\left(\psi_{\eta} \nabla^{2} \psi_{\xi}-\psi_{\xi} \nabla^{2} \psi_{\eta}+\widetilde{F}\right) d \zeta=\psi\left(\zeta_{0}\right)+\int_{a-1<\xi<a} \psi L^{*}\left(\mu K\left(\zeta_{0}, \zeta\right)\right) d \zeta \quad(d \zeta=d \xi d \eta),
$$

or equivalently 


$$
\psi\left(\zeta_{0}\right)=\int_{S_{a}}\left(K_{\eta} \psi_{\xi}-K_{\xi} \psi_{\eta}\right) \nabla^{2} \psi d \zeta+T\left(\zeta_{0}\right) \quad\left(\xi_{0}>a\right)
$$

where

$$
T\left(\zeta_{0}\right)=\int_{a-1<\xi<a}\left[\psi L^{*}(\mu K)+\left\{\psi_{\xi}(\mu K)_{\eta}-\psi_{\eta}(\mu K)_{\xi}\right\} \nabla^{2} \psi\right] d \zeta+\int_{a-1<\xi} \mu K\left(\zeta_{0}, \zeta\right) \tilde{F} d \zeta
$$

LEMma 6.5. Define

$$
\left(A^{\alpha \beta} \phi\right)\left(\zeta_{0}\right)=\int_{S_{a}} \phi(\zeta) \nabla^{2} \psi(\zeta) \nabla_{0}^{\alpha} \nabla^{\beta} K\left(\zeta_{0}, \zeta\right) d \zeta, \quad \zeta_{0} \in S_{a},|\alpha|=|\beta|=1
$$

for all $\phi$ in $C_{1}\left(\overline{\bar{S}}_{a}\right)$ or $E_{1}\left(S_{a}\right)$. Then

(a) $\left\|A^{\alpha \beta} \phi\right\|_{C_{1}} \leqslant \varepsilon(\alpha)\|\phi\|_{C_{3}}$,

(b) $\left\|A^{\alpha \beta} \phi\right\|_{E_{3}} \leqslant \varepsilon(a)\|\phi\|_{E_{1}}$,

and $\varepsilon(a) \rightarrow 0$ as $a \rightarrow \infty$.

Proof. The proof of (a) appears in the proof of Lemma 4.3 in [5], and so we shall prove only (b). Let $\phi \in E_{1}$. Then $A^{\alpha \beta} \phi \in C_{1}\left(\widetilde{S}_{a}\right)$ by (a), and we estimate $\left\|A^{\alpha \beta} \phi\right\|_{E_{1}}$ in three stages. Set $W\left(\zeta_{0}, \zeta\right)=\nabla_{0}^{\alpha} \nabla^{\beta} K\left(\zeta_{0}, \zeta\right)$.

(i) $\quad\left|\int_{\xi_{0}+2<\xi} \phi \nabla^{2} \psi W\left(\zeta_{0}, \zeta\right) d \zeta\right| \leqslant$ const. $\|\phi\|_{E_{1}} \int_{\xi_{0}+2<\xi} \xi^{-\gamma}\left|\nabla^{2} \psi\right| e^{-c\left(\xi-\xi_{0}\right)} d \zeta$ $\leqslant$ const. $\|\phi\|_{E_{1}} \xi_{0}^{-\gamma}\left\|\nabla^{2} \psi\right\|_{0,2, S_{a}}$

and $\left\|\nabla^{2} \psi\right\|_{0,2, s_{a}} \rightarrow 0$ as $a \rightarrow \infty$ since $\psi \in \stackrel{\circ}{W}_{2}^{2}(S)$.

(ii)

$$
\begin{aligned}
& \left|\int_{\xi_{0}-2<\xi<\xi_{0}+2} \phi \nabla^{2} \psi W\left(\zeta_{0}, \zeta\right) d \zeta\right| \\
& \quad \leqslant \text { const. }\|\phi\|_{E_{1}} \int_{\xi_{0}-2<\xi<\xi_{0}+2} \xi^{-\gamma\left|\nabla^{2} \psi\right|\left(1+|\log | \xi-\xi_{0} \|^{\prime}\right) d \zeta} \\
& \quad \leqslant \text { const. }\|\phi\|_{E_{1}} \xi_{0}^{-\gamma\left\|\nabla^{2} \psi\right\|_{0,2, S_{a}}}
\end{aligned}
$$

(iii) $\left|\int_{a<\xi<\xi_{0}-2} \phi \nabla^{2} \psi W\left(\zeta_{0}, \zeta\right) d \zeta\right| \leqslant$ const. $\|\phi\|_{E_{1}}\left\|\nabla^{2} \psi\right\|_{C_{1}} \int_{a}^{\xi_{0}-2} \xi^{-\gamma} e^{-b\left(\xi_{0}-\xi\right)} d \xi$,

and an integration by parts gives

$$
\begin{aligned}
\int_{a}^{\xi_{0}-2} \xi^{-\gamma} e^{-b\left(\xi_{0}-\xi\right)} d \xi & \leqslant \frac{1}{b} e^{-2 b}\left(\xi_{0}-2\right)^{-\gamma}+\frac{\gamma}{b} \int_{a}^{\xi_{0}-2} \xi^{-1-\gamma} e^{-b\left(\xi_{0}-\xi\right)} d \xi \\
& \leqslant \text { const. } \xi_{0}^{-\gamma}
\end{aligned}
$$

where the constant is independent of $a$ and $\xi_{0}$. Use of this in (6.24) gives 


$$
\left|\int_{a<\xi<\xi_{0}-2} \phi \nabla^{2} \psi W\left(\zeta_{0}, \zeta\right) d \zeta\right| \leqslant \text { const. }\|\phi\|_{E_{1}} \xi_{0}^{-\gamma}\left\|\nabla^{2} \psi\right\|_{C_{1}}
$$

and. $\left\|\nabla^{2} \psi\right\|_{c_{1}} \rightarrow 0$ as $a \rightarrow \infty$ by Theorem 6.2.

Q.E.D.

If we use Lemma 6.4 and arguments similar to those in Lemma 4.4 of [5], then a straightforward (but tedious) argument gives

Lемма 6.6. Let $T$ be as in (6.23); then $\nabla^{\alpha} T \in E_{1}\left(S_{a}\right)$, for $|\alpha| \leqslant 1$.

The proof of the following theorem is almost identical to that of Theorem 4.5 in [5].

THЕовем 6.7. If $|a|$ is sufficiently large, then $\nabla^{\alpha} \psi \in E_{1}\left(S_{a}\right)$ for $|\alpha|=1$.

Proof. Using the representation formula (6.23) for $\psi$ and the definition of $A^{\alpha \beta}$ and $T$ in Lemma 6.5 and (6.23), respectively, we have for $\zeta \in S_{a}$ and $|\alpha|=1$,

$$
\nabla^{\alpha} \psi(\zeta)=\sum_{|\beta|=|\alpha|=1} a_{\alpha \beta \sigma}\left(A^{\alpha \beta} \nabla^{\sigma} \psi\right)(\zeta)+\nabla^{\alpha} T(\zeta)
$$

for certain constants $\alpha_{\alpha \beta \sigma}= \pm 1$ or 0 . If we let $g=\nabla \psi$ and $h=\nabla T$, then (6.25) can be written as

$$
g-A g=h .
$$

For a Banach space $B$, let $\mathcal{L}(B)$ denote the Banach space of bounded linear maps $B \rightarrow B$. By Lemma 6.6, we have $h=\nabla T \in E_{2}$, and standard theory shows that the equation $r-A r=h$, for $h \in E_{2}$, has a unique solution

(a) in $C_{2}$, if $a$ is so large that $\|A\|_{\mathfrak{L}\left(C_{2}\right)}<1$,

(b) in $E_{2}$, if $a$ is so large that $\|A\|_{{\mathcal{C}\left(E_{2}\right)}}<1$,

and by Lemma 6.5, the number $a$ can, and will, be so chosen. Since $E_{2} \subset C_{2}$, these two solutions are identical. Theorem 6.2 shows that $\nabla \psi \in C_{2}$, and so we have $\nabla \psi \in E_{2}$. Q.E.D.

By continuity on the set $\{\tilde{a} \leqslant \xi \leqslant a\}$, we have $\nabla \psi \in E_{2}\left(S_{a}\right)$ for all $a \geqslant \tilde{a}$. If we apply this result to $(6.20)$, then it follows that $L \psi \in E_{1}\left(S_{a}\right)$ for all $a \geqslant \tilde{a}$, and one can then prove

Theовем 6.8. Assume that

$$
|x(\xi, \eta)|,|\lambda(\xi, \eta)| \leqslant \widetilde{C}-\gamma, \quad \xi>1,
$$

for constants $\tilde{C}, \gamma>0$. Then the perturbation stream function $\psi$ satisfies

$$
\left|\nabla^{\alpha} \psi(\xi, \eta)\right| \leqslant \text { const. } \xi^{-\gamma}, \quad \xi>1,
$$

for each multi-index a with $|\alpha| \leqslant 3$. 
If $x$ and $\lambda$ decay exponentially at infinity (as is the case when sufficiently far upstream, and sufficiently far downstream, the channel walls $\Gamma_{+}$and $\Gamma_{-}$are exactly parallel), then one can use the methods in [5] to prove corresponding results for various derivatives of $\psi$.

\section{Appendix A. On the conformal mapping of a strip onto a channel}

\section{A.1. Examples of maps $\boldsymbol{F}$}

Notation. Throughout this Appendix $a, b, c, k, m, \beta$ and $\gamma$ will denote real nonnegative constants; in addition to the complex variables $z=x+i y$ and $\zeta=\xi+i \eta$, we shall use $w=u+i v$ and $t=r+i s$; and the symbols $u, v, w, G, \psi, \ldots$ do not have the meanings assigned to them in the main text. For functions $f$ of one variable, $f^{(n)}$ denotes the $n$th derivative. 'Symmetry' means symmetry with respect to a streamwise axis.

In examples (i) to (vii), channels are of type $I^{\prime}$ (Definition 5.1) whenever they are of type I, and type $I^{\prime}$ will not be mentioned there. In examples (i) to (iv), the function $F$ will be defined not merely on $\bar{S}$, but as a conformal, one-to-one map on an open set containing $\bar{S}$.

(i) A family of asymmetrical channels. Let the transformation $z=F(\zeta)$ be

$$
z=\frac{1}{m}(\zeta+i b)^{m}, \quad 1 \leqslant m \leqslant 2, \quad b>1, \quad|\zeta+i b|>0, \quad 0<\arg (\zeta+i b)<2 \pi / m
$$

The asymmetrical channels of the title are those with $m>1$; the case $m=3 / 2, b=3 / 2$ was shown in Fig. 1. For $|\xi| \rightarrow \infty$ in $S$, we have

$$
\begin{gathered}
h(\zeta)=|d z / d \zeta| \sim|\xi|^{m-1} \\
|\nabla \log h(\zeta)|=|\varkappa(\zeta)-i \lambda(\zeta)|=\frac{\left|d^{2} z / d \zeta^{2}\right|}{|d z| d \zeta \mid} \sim(m-1)|\xi|^{-1}
\end{gathered}
$$

It follows that $\Omega=F(S)$ is of type I if $m>3 / 2$, of type II for all $m \in[1,2]$, and of type III if $1 \leqslant m \leqslant 3 / 2$.

(ii) A family of symmetrical channels with boundaries $y \sim \pm$ const. $|x|^{k}, 0 \leqslant k<1$. (The symbol $\sim$ of asymptotic equality refers to $|x| \rightarrow \infty$.) Now let $F$ be given by

$$
\left.\begin{array}{cl}
z=c \zeta\left(\zeta^{2}+b^{2}\right)^{\frac{1}{2}(m-1)}, & m \geqslant 1, b>1, c>0, \\
<\arg (\zeta+i b)<3 \pi / 2, & -3 \pi / 2<\arg (\zeta-i b)<\pi / 2 .
\end{array}\right\}
$$

These restrictions of $\arg (\zeta \pm i b)$ ensure that $\arg \left(\zeta^{2}+b^{2}\right)=0$ on the real axis and on the line segment $\xi=0,|\eta|<b$; also, $d z / d \zeta>0$ on the real axis. 


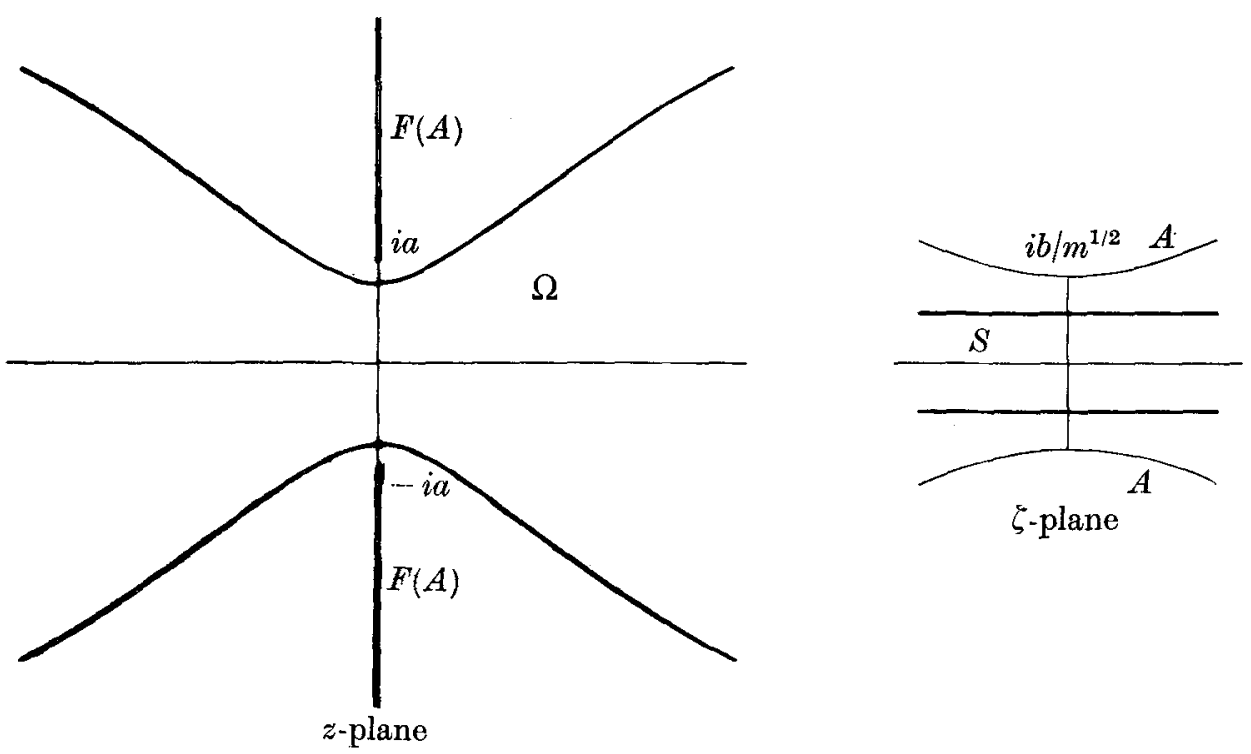

Fig. 4. The case $m=3, b=3$ of the mapping in example (ii).

The condition $b>1$ is not enough, in general, to make $F: \bar{S} \rightarrow \bar{\Omega}$ a diffeomorphism; we have $d z / d \zeta=0$ at $\zeta= \pm i b / m^{\frac{1}{2}}$, and denote the images of these points by $z= \pm i a$. Therefore we require at least that $b>m^{\frac{1}{2}}$. To discover the largest set on which the map (A.2) is one-to-one, we trace the inverse image, say $A$, of the lines $x=0 \pm,|y| \geqslant a$, and use a classical theorem ([27], p. 201) to the effect that, under mild restrictions, a function holomorphic in a bounded, simply-connected open set $B$ is one-to-one on $\bar{B}$ if it is oneto-one on $\partial B$. It is sufficient to consider that part of the first quadrant of the $\zeta$-plane which lies below the upper component of $A$; one can prove that $A$ does not cross itself, $h$ vanishes on the upper component of $A$ only at $\zeta=i b / m^{\frac{1}{2}}$, and the nature of $F$ as $|\zeta| \rightarrow \infty$ is clear. The theorem then shows that $F$ is one-to-one (and it is certainly conformal) on the open set between the two components of $A$. For $m=2$ or 3 , the set $A$ is a hyperbola:

$$
\eta^{2}=\xi^{2}+\frac{1}{2} b^{2} \quad \text { if } m=2, \quad \text { or } \quad \eta^{2}=\frac{1}{3}\left(\xi^{2}+b^{2}\right) \quad \text { if } m=3
$$

for arbitrary values of $m$, exploration of $A$ is an arduous affair. It turns out that, if $m \leqslant 5$, then $\eta^{2} \geqslant b^{2} / m$ on $A$, but, if $m>5$, then $\eta^{2}<b^{2} / m$ on a part of $A$. We choose $b$ so large that $\eta^{2}>1$ on $A$; for $m \leqslant 5$ this means $b>m^{\ddagger}$, for $m>5$ such a choice is possible.

Fig. 4 shows the case $m=3, b=3$. Since $x \sim c \xi^{m}$ and $y \sim c m \xi^{m-1} \eta$ as $\xi \rightarrow \infty$ with $\eta$ fixed, the exponent in the title is $k=(m-1) / m$. Essentially as in (i), $F(S)$ is of type $\mathrm{I}$ if $m>3 / 2$, of type II for all $m \geqslant 1$, and of type III if $1 \leqslant m \leqslant 3 / 2$. 


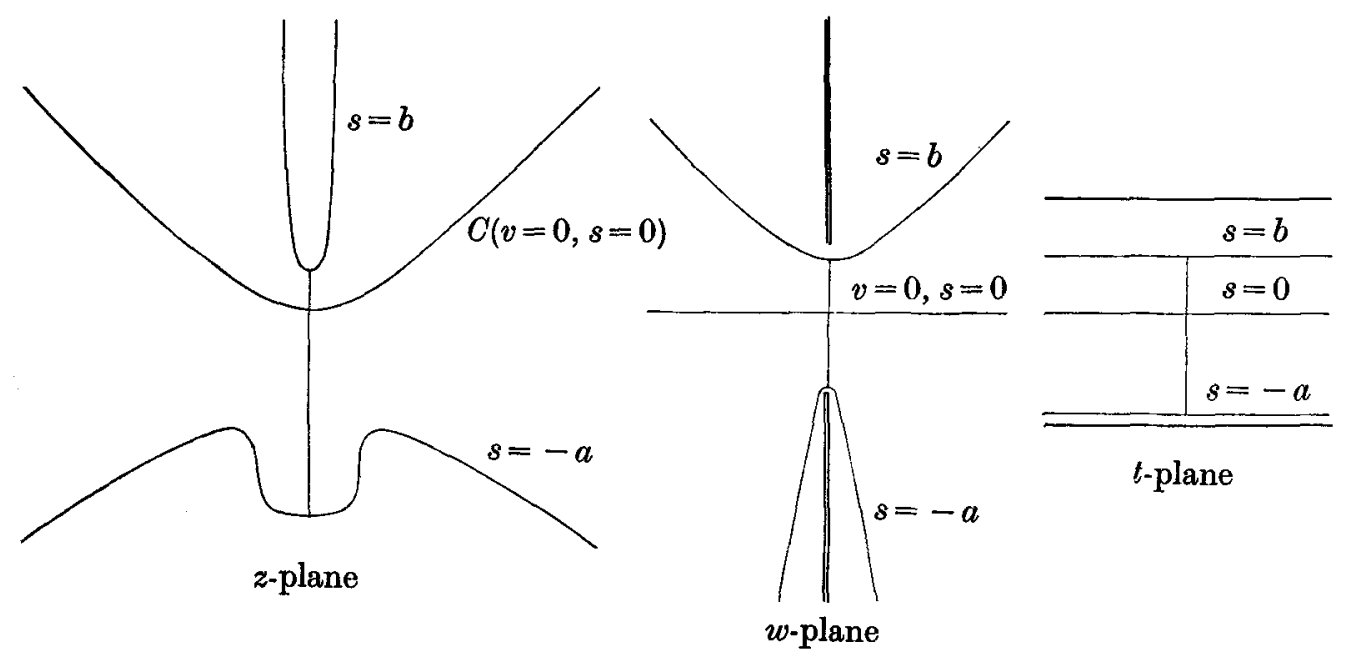

Fig. 5. The bending transformation in example (iv). The case drawn is $\gamma=\pi / 4$.

(iii) Channels bounded by hyperbolae. The well known transformation

$$
z=\sinh \beta \zeta, \quad 0<\beta<\pi / 2, \quad \zeta \in \mathbf{R} \times(-\pi / 2 \beta, \pi / 2 \beta),
$$

maps $S$ onto the channel $\Omega$ between the branches (components) of the hyperbola

$$
\partial \Omega:\left(\frac{y}{\sin \beta}\right)^{2}=\left(\frac{x}{\cos \beta}\right)^{2}+1 .
$$

Such a channel is of type $I$ and not of type $I ; x(\zeta)-i \lambda(\zeta)=\beta \tanh \beta \zeta \rightarrow \pm \beta$ as $\xi \rightarrow \pm \infty$.

(iv) Bending an initially symmetrical channel. The transformation (Fig. 5)

for which

$$
z\left(w_{0}\right)=\int_{0}^{w_{0}} \exp \left\{\frac{i \gamma w}{\left(w^{2}+1\right)^{1 / 2}}\right\} d w, \quad 0<\gamma<\pi / 2,
$$

$$
\left.\begin{array}{rl}
\log \frac{d z}{d w}= & \frac{i \gamma w}{\left(w^{2}+1\right)^{1 / 2}}, \\
& -\pi / 2<\arg (w+i)<3 \pi / 2, \quad-3 \pi / 2<\arg (w-i)<\pi / 2,
\end{array}\right\}
$$

bends the axis $v=0$ into a curve $C: x=x(u, 0), y=y(u, 0)$ such that the tangent to $C$ makes an angle $\gamma u /\left(u^{2}+1\right)^{\frac{1}{2}}$ with the horizontal. If $\Omega_{0}$ is a channel in the $w$-plane that is symmetrical about the axis $v=0$, then the transformation bends $\Omega_{0}$ correspondingly into a new channel $\Omega_{\gamma}$ in the $z$-plane, provided that $\bar{\Omega}_{0}$ lies in an open set on which the map (A.3) is one-to-one.

10-792901 Acra mathematica 144. Imprimé le 13 Juin 1980 
Denote the transformation (A.3) by $z=G(w)$; then, as $|w| \rightarrow \infty$ in the cut $w$-plane, $\left|G^{\prime}(w)\right|=1+O\left(w^{-2}\right)$ and $\left|G^{\prime \prime}(w)\right|=O\left(w^{-3}\right)$. Let $w=H(\zeta)$ map $S$ onto $\Omega_{0}$ in the standard manner; then $F=G \circ H$ maps $S$ onto $\Omega_{\gamma}$.

It follows that, if $H^{\prime 2}=o\left(H^{3} H^{\prime \prime}\right)$, then $\left|F^{\prime}(\zeta)\right| \sim\left|H^{\prime}(\zeta)\right|$ and $\left|F^{\prime \prime}(\zeta)\right| \sim\left|H^{\prime \prime}(\zeta)\right|$ as $|\zeta| \rightarrow \infty$ in $S$, so that $\Omega_{\gamma}$ and $\Omega_{0}$ are of the same type.

This condition is amply satisfied if $\Omega_{0}$ is one of the channels in (ii) with $m>1$. If $m=1$ in (ii), so that $\Omega_{0}$ is the strip $\mathbf{R} \times(-c, c)$, then our estimates of $G^{\prime}$ and $G^{\prime \prime}$ show that $\Omega_{y}$ is, like $\Omega_{0}$, of type III.

We must find a large domain in which $G$ is one-to-one. It is helpful to introduce the auxiliary transformation

Then

$$
w=\sinh t \quad(t=r+i s), \quad-\pi / 2<s<\pi / 2 .
$$

$$
z\left(t_{0}\right)=\int_{0}^{t_{0}} \exp (i \gamma \tanh t) \cosh t d t
$$

We seek the largest horizontal strip in the $t$-plane on which $|\arg d z / d t|<\pi / 2$; denote it by $S_{a b}=\mathbf{R} \times(-a, b)$, where $a, b \in(0, \pi / 2)$. Then the map (A.4) is one-to-one on $S_{a b}$, by the theorem ([27], p. 201) cited in (ii) and the fact that $|d z / d t| \neq 0$ on $\partial S_{a b}$. Note that in the $w$-plane the image of $S_{a b}$ is a domain between branches of hyperbolae. To calculate $a$ and $b$, it is sufficient to take $r \geqslant 0$. One finds that $\arg d z / d t>-\pi / 2$ on $[0, \infty) \times(-\pi / 2, \pi / 2)$, so that only the condition $\arg d z / d t<\pi / 2$ determines $\alpha$ and $b$. Define $\alpha=\alpha(\gamma)$ by: $\sin 2 \alpha=2 \gamma / \pi$ and $\alpha \in(\pi / 4, \pi / 2)$. A calculation shows that $a \in(\alpha, \pi / 2)$, that $a$ is the solution of a certain unpleasant equation, and that $b=\pi / 2-\gamma<\alpha(\gamma)$.

Finally, we note that (A.4) maps the strip $\mathbf{R} \times(-\beta, \beta)$ into a bent form $\Omega_{\gamma}$ of a hyperbolic channel $\Omega_{0}$ as in (iii), provided that $\beta<\min \{a, b\}$. The condition $\beta+\gamma<\pi / 2$ is necessary and sufficient for this. The channels $\Omega_{\gamma}$ and $\Omega_{0}$ are both of type $I$ and not of type II.

\section{A.2. End maps}

By a downstream end map $E$ for a channel $\Omega=F(S)$ we mean an asymptotic approximation to $F$; that is, $F(\zeta) \sim E(\zeta)$ on $\bar{S}$ as $\xi \rightarrow \infty$. Upstream end maps are defined similarly for $\xi \rightarrow-\infty$, but we shall mainly consider the former case, and shall then omit the word downstream. The type of a channel is implied by sufficiently accurate upstream and downstream end maps. Just as the exact maps $F$ in (i) to (iv) were defined as conformal, one-to-one functions on open sets containing $\bar{S}$, so we shall define end maps $E$, wherever possible, as such functions on open sets containing the closure $\bar{S}_{a}$ of a half-strip $S_{a}=(a, \infty) \times(-1,1)$. 
In ( $v)$ to (vii), we construct functions $E$ which we hope to be useful end maps for the channels $\Omega_{f}$ defined by (1.6); for these channels, the exact mapping functions $F$ are, of course, unknown, since $f$ has not been specified completely. Theorem A.3 and its corollaries will show that these functions $E$ are indeed sufficiently accurate end maps, with room to spare. The downstream part of a channel $\Omega_{f}$ is now denoted by

$$
\Omega_{k}=\left\{z \mid x>\text { const. } \geqslant 1, \quad-A x^{k}<y<A x^{k}\right\}, \quad A>0, k \geqslant 0 .
$$

In (viii) and (ix), we characterize new families of channels by means of end maps that are sufficiently accurate by hypothesis, in order to display certain admissible (and certain inadmissible) oscillations of the boundary $\partial \Omega$.

(v) An end map for $\Omega_{k}, k<1$, and for the channels in (ii). The obvious choice of transformation $z=E(\zeta)$ is

$$
z=c \zeta^{m}, \quad m \geqslant 1, c>0, \quad|\zeta|>0,-\pi / m<\arg \zeta<\pi / m
$$

The domain of $E$ is the sector indicated in (A.6), and contains the closure $\bar{S}_{a}$ of a half-strip if $a>0$ when $1 \leqslant m \leqslant 2$, or if $a>\cot \pi / m$ when $m \geqslant 2$. We have

$$
x=c \xi^{m} P_{1}(\eta / \xi), \quad y=c m \xi^{m-1} \eta P_{2}(\eta / \xi)
$$

where $P_{j}(t)$ denotes a function that may be expanded as a series $1+$ const. $t^{2}+\ldots$ in powers of $t^{2}$, with leading term 1 and radius of convergence 1 .

To make the images of the lines $\eta= \pm 1$ close to $\partial \Omega_{k}$, we choose $m$ so that $k=(m-1) / m$ and $c$ so that $c^{1-k}=A / m$. To examine the precise difference between the inverse image $E^{-1}\left(\partial \Omega_{k_{k}}\right)$ and the lines $\eta= \pm 1$, we consider the upper boundary component, define

$$
\Phi(\xi, \eta)=\left(y-A x^{k}\right) / c m \xi^{m-1}=\eta P_{2}(\eta \mid \xi)-P_{1}(\eta / \xi)^{k},
$$

and seek the solution of $\Phi(\xi, \eta)=0$. Since $\Phi(\xi, 1)=O\left(\xi^{-2}\right)$ and $\Phi_{\eta}(\xi, 1)=1+O\left(\xi^{-2}\right)$, it is not difficult (see (vii)) to define, for $a$ sufficiently large, a contraction mapping that implies existence of a solution $\eta=1+f(\xi), \xi \geqslant a$, such that $f^{(n)}(\xi)=O\left(\xi^{-2-n}\right), n=0,1, \ldots$.

The contraction mapping also implies uniqueness of $f$ in a certain ball, but a stronger uniqueness result is available. Recall that $\Omega_{k} \subset \Omega_{f}$, and observe that, in the z-plane and for sufficiently large values of $a$, each component of $\partial \Omega_{f}$ intersects exactly once each of the arcs onto which $E$ maps the line segments $\xi=$ const. $\geqslant a,|\arg \zeta| \leqslant \pi / 2 m$.

For the channels in (ii), the exact mapping function $F$ is known; it is clear that $F(\zeta) \sim E(\zeta)$ on $\bar{S}$ as $\xi \rightarrow \infty$, and it is easily verified that $\operatorname{Im}\left(E^{-1} \circ F\right)(\xi+i)$ has the properties just established for $1+f(\xi)$. 


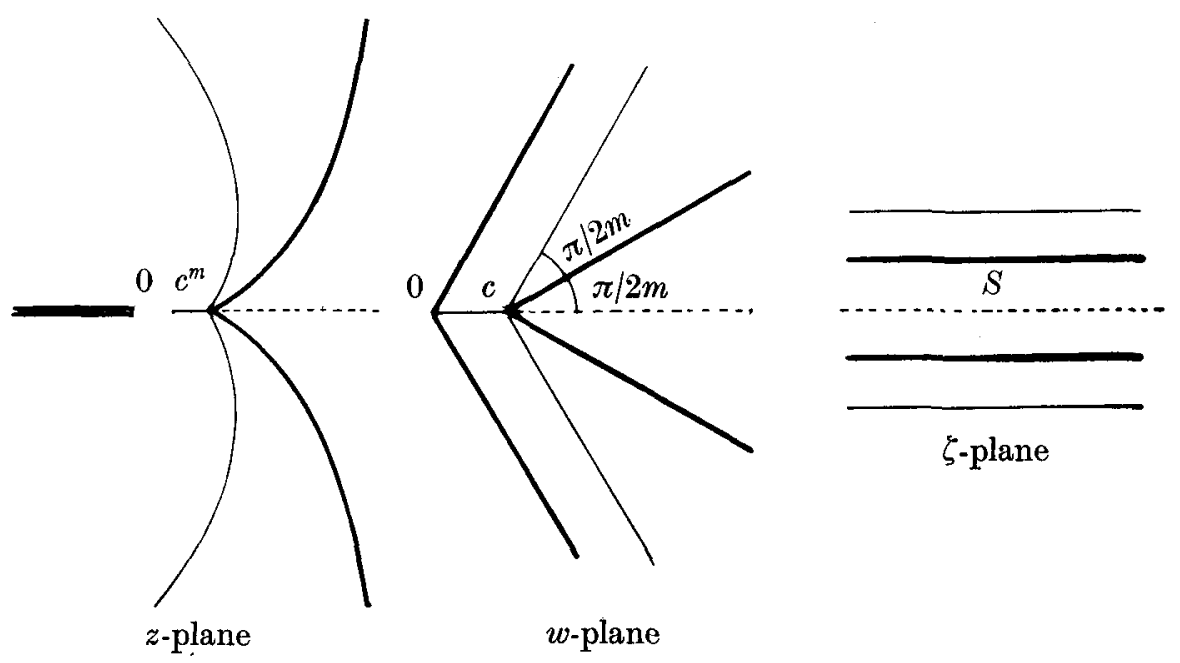

Fig. 6. Item (vii): an end map for $\Omega_{k}, k>1$.

(vi) An end map for $\Omega_{k}, k=1$, and for the channels in (iii). In (A.5), let $A=\tan \beta$, $0<\beta<\pi / 2$; an appropriate map $E$ is given by

$$
z=\frac{1}{2} e^{\beta \zeta}, \quad \zeta \in \mathbf{R} \times(-2,2) .
$$

This is exact for $\Omega_{k}$, and has exponentially small error for the hyperbolic channels in (iii).

(vii) An end map for $\Omega_{k}, k>1$. In this case we define $E$ by combining the transformations (Fig. 6)

and

$$
z=w^{m}, \quad m>1, \quad-\pi / m<\arg w<\pi / m
$$

$$
w=\exp \frac{\pi \zeta}{2 m}+c, \quad c>0, \quad \zeta \in \mathbf{R} \times(-2,2)
$$

Choose $m$ so that $k=m /(m-1)$, and in (A.5) set $A=b^{-k}$. The notation $p_{j}=\pi(m-j) / 2 m$, $j=0,1,2$, will be convenient; we emphasize that $p_{0}=\pi / 2$ and $p_{0} / p_{1}=k$.

As in $(\nabla)$, we examine the inverse image $E^{-1}\left(\partial \Omega_{k}\right)$. Eq. (A.7) imply that

$$
z=e^{p_{0} \zeta}+m c e^{p_{1} \zeta}+m(m-1) c^{2} e^{p_{2} \zeta} \int_{0}^{1}(1-\tau)\left\{1+\tau c \exp \left(-\frac{\pi \zeta}{2 m}\right)\right\}^{m-2} d \tau,
$$

and it follows that

where

$$
\Phi(\xi, \eta)=x-b y^{1 / k}=\phi(\xi, \eta)+R(\xi, \eta)
$$

$$
\phi(\xi, \eta)=e^{p_{0} \xi} \cos p_{0} \eta+e^{p_{1} \xi}\left\{m c \cos p_{1} \eta-b\left(\sin p_{0} \eta\right)^{p_{1} / p_{0}}\right\},
$$


while $R$ and all its derivatives are $O\left(e^{p_{2} \xi}\right)$. We choose $c=b / m \cos p_{1}$, noting that $0<p_{1}<\pi / 2$; then

$$
\phi(\xi, 1)=0 \text { and } \Phi_{\eta}(\xi, 1)=-p_{0} e^{p_{0} \xi}+O\left(e^{p_{1} \xi}\right)
$$

The solution of $\Phi(\xi, \eta)=0$ will have the form $\eta=1+f(\xi)$; to house $f$, we introduce a Banach space, with weight function $\varrho$, of functions having bounded continuous derivatives to order $q$ on $[a, \infty)$ :

$$
C_{a, \varrho}^{a}=\left\{g \in C^{q}([a, \infty))\left|\|g\|_{\alpha, a, \varrho}=\sup _{\xi \geqslant a, n \leqslant Q} \varrho(\xi)\right| g^{(n)}(\xi) \mid<\infty\right\}
$$

where $n \leqslant q$ means $n=0,1, \ldots, q$, and $\varrho(\xi)=\exp \left\{\left(p_{0}-p_{2}\right) \xi\right\}=\exp (\pi \xi / m)$. Our task is to find a solution in $C_{a, \varrho}^{q}$ of $T f=f$, where

$$
(T g)(\xi)=-\frac{1}{\Phi_{\eta}(\xi, 1)}\left\{R(\xi, 1)+g(\xi)^{2} \int_{0}^{1}(1-\tau) \Phi_{\eta \eta}(\xi, 1+\tau g(\xi)) d \tau\right\}
$$

Fix the integer $q$; then there exist numbers $a_{0}$ and $r_{0}$ such that

$$
\left\|R(., 1) / \Phi_{\eta}(., 1)\right\|_{a, a_{0}, \ell} \leqslant r_{0},
$$

and we let $B=B\left(0,2 r_{0}\right)$ denote the ball in $C_{a_{.} \varrho}^{q}$ with centre the origin and radius $2 r_{0}$. It is a routine matter to show that, if $a \geqslant a_{0}$ is chosen sufficiently large, $T$ is a contraction map on the complete metric space $\bar{B}$. Then $T$ has a unique fixed point $f$ in $\bar{B}$, so that

$$
f^{(n)}(\xi)=O\left(e^{-\pi \hat{\xi} / m}\right), \quad n=0,1, \ldots, q .
$$

We also have a stronger uniqueness result, as in (v).

(viii) Ends of type $I$ with oscillatory boundaries. Consider the transformation

$$
\left.\begin{array}{c}
z=\frac{1}{m} \zeta^{m}+\frac{2 c}{b} \zeta^{a} \exp \left(-b \zeta^{b-1}\right) \cos \zeta^{b}+R(\zeta) \\
m>3 / 2, \quad a \geqslant 0, \quad 1<b<3
\end{array}\right\}
$$

where $\arg \zeta=0$ on the positive real axis, and $R^{(n)}(\zeta)=o\left(\xi^{m-n}\right)$ uniformly on $\bar{S}$, for $n=0,1,2$ and $\xi \rightarrow \infty$. We have chosen $m>3 / 2$ in order to have a downstream end of type $I$ when $c=0 ; b>1$ because rapid oscillations are of interest; and $b<3$ because otherwise $\exp \left(-b \xi^{b-1}\right)$ would have to be replaced by a longer expression for the sake of certain conclusions. Henceforth $c>0$.

We note that

$$
\begin{aligned}
2 \exp \left(-b \zeta^{b-1}\right) \cos \zeta^{b}= & \exp \left[-b \xi^{b-1}(1+\eta)+O\left(\xi^{b-3}\right)+i\left\{\xi^{b}+O\left(\xi^{b-2}\right)\right\}\right] \\
& +\exp \left[-b \xi^{b-1}(1-\eta)+O\left(\xi^{b-3}\right)-i\left\{\xi^{b}+O\left(\xi^{b-2}\right)\right\}\right],
\end{aligned}
$$


so that the oscillatory terms in (A.8) are exponentially small within $S$, while on $\partial S$

$$
\left.\begin{array}{l}
x=\frac{1}{m} \xi^{m}+\frac{c}{b} \xi^{a} \cos \left\{\xi^{b}+\frac{1}{2} b(b-1) \xi^{b-2}+\ldots\right\}+\ldots, \\
y= \pm \xi^{m-1} \mp \frac{c}{b} \xi^{a} \sin \left\{\xi^{b}+\frac{1}{2} b(b-1) \xi^{b-2}+\ldots\right\}+\ldots
\end{array}\right\}
$$

The following results are implied by the formulae for $d z / d \zeta$ and $d^{2} z / d \zeta^{2}$.

In order that the mapping (A.8) be one-to-one on $\bar{S}$ for $\xi$ sufficiently large (henceforth this qualifying phrase is to be understood), we demand that

$$
a+b \leqslant m, \quad 0<c<1 .
$$

(The condition $a+b<m$ would allow any value of $c$, but would be less informative.) Then $h(\zeta) / \xi^{m-1}$ is bounded between positive constants on some half-strip $S_{d}$, so that $1 / h \in L_{2}\left(S_{d}\right)$. A calculation shows that $|\nabla(1 / h)| \in L_{2}\left(S_{d}\right)$ for some $d$ if and only if

$$
2 a+3 b<4 m-2,
$$

which is implied by (A.10) when $m>2$, but not when $m \leqslant 2$. Accordingly, as regards its downstream end, a channel defined by (A.8), and hence having a boundary as in (A.9), is of type I whenever (A.10) and (A.11) hold. When $m=2, a=0$ and $b=2(a+b=m$, $2 a+3 b=4 m-2)$ the corresponding channel is not of type $I$.

Now consider membership of the set $I^{\prime}$. The first additional condition, bounding the ratio $h(\zeta) / h\left(\xi_{0},-1\right)$ in a neighbourhood on $S$ of the point $\left(\xi_{0},-1\right)$, is satisfied because $h(\zeta) / \xi^{m-1}$ lies betwe en positive constants. The second, that $|\nabla(1 / h)(\zeta)| \rightarrow 0$ uniformly on $S$ as $\xi \rightarrow \infty$, requires that $a+2 b<2 m$. Comparing this with (A.10) and (A.11) (most easily by graphs in the $a b$-plane of the three lines in question), we conclude that, as regards their downstream ends, the present channels of type $I$ are also of type $I^{\prime}$ whenever $m \leqslant 2$ or $a>0$; but, if $m>2, a=0$ and $b=m$, there results a channel of type $I$ that is not of type $I^{\prime}$.

(ix) Ends of type III with oscillatory boundaries. Since the definition of type III leads to Poiseuille flow, which is undirectional in its exact setting, we can expect only feeble oscillations to be admissible in this case. If we try to proceed with the formula in (A.8), now taking $1 \leqslant m \leqslant 3 / 2$ and $0<b<3$, the condition $|\nabla h|=\left|d^{2} z / d \zeta^{2}\right| \in L_{q}$ for some $q>2$ (which is weakest in the present case when $q=\infty$ ) requires that $a+2 b-2 \leqslant 0$, hence $b \leqslant 1$. There is then no exponential decay within $S$, and so the condition $|\nabla \log h| \in L_{2}$ requires that

$$
a+2 b<m+\frac{1}{2} \leqslant 2,
$$


since $m \leqslant 3 / 2$. Thus $b<1$. Accordingly, we are forced to consider

$$
\left.\begin{array}{l}
z=\frac{1}{m} \zeta^{m}+\frac{2 c}{b} \zeta^{a} \cos \zeta^{b}+R(\zeta), \\
1 \leqslant m \leqslant \frac{3}{2}, \quad a \geqslant 0, \quad 0<b<1,
\end{array}\right\}
$$

where $\arg \zeta$ and $R(\zeta)$ are as for (A.8). The boundary $\partial \Omega$ has the different form

$$
\begin{gathered}
x=\frac{1}{m} \xi^{m}+\frac{2 c}{b} \xi^{a} \cos \left(\xi^{b}+\ldots\right)+\ldots, \\
y= \pm \xi^{m-1} \mp 2 c \xi^{a+b-1} \sin \left(\xi^{b}+\ldots\right)+\ldots,
\end{gathered}
$$

and in place of (A.10) we demand that

$$
a+b \leqslant m, \quad 0<c<\frac{1}{2}
$$

It turns out that (A.12) and (A.13) are sufficient (and virtually necessary) for membership of the set III.

\section{A.3. On pertubations of end maps}

In this section our main tool will be the Green function $K\left(\zeta_{0}, \zeta\right)$ of the Dirichlet problem for the Laplace operator $-\nabla^{2}$ in the half-strip $S_{a}=(a, \infty) \times(-1,1)$; for fixed $\zeta_{0} \in S_{a}$, this is the solution of

$$
-\nabla^{2} K=\delta\left(\zeta-\zeta_{0}\right) \quad \text { in } S_{a},\left.\quad K\right|_{\zeta \Subset \partial S_{a}}=0,
$$

where $\delta$ denotes the Dirac distribution. If $S$ replaces $S_{a}$, the corresponding Green function $G$ is

$$
\begin{gathered}
G\left(\zeta_{0}, \zeta\right)=\operatorname{Re} \frac{1}{2 \pi} \log \frac{\sinh \frac{1}{2}\left(Z-\bar{Z}_{0}\right)}{\sinh \frac{1}{2}\left(Z-Z_{0}\right)} \\
=\frac{1}{4 \pi} \log \left\{1+\frac{2 \sin Y \sin Y_{0}}{\cosh \left(X-X_{0}\right)-\cos \left(Y-Y_{0}\right)}\right\} \\
=\frac{2}{\pi} \sum_{n=1}^{\infty} \frac{1}{2 n} e^{-n\left|X-X_{0}\right|} \sin n Y \sin n Y_{0},
\end{gathered}
$$

where $Z=X+i Y=\pi(\zeta+i) / 2$, so that $Z \in \mathbf{R} \times[0, \pi]$. With the notation $G\left(\zeta_{0}, \zeta\right)=$ $\tilde{G}\left(\left|X-X_{0}\right|, Y, Y_{0}\right)$, the Green function for the half-strip $S_{a}$ is

$$
K\left(\zeta_{0}, \zeta\right)=\tilde{G}\left(\left|X-X_{0}\right|, Y, Y_{0}\right)-\tilde{G}\left(X+X_{0}-\pi a, Y, Y_{0}\right)
$$

where $\zeta_{0}, \zeta \in \bar{S}_{a}$. As an introduction to the more elaborate result which follows, we have 
THEOREM A.1. Let $\Omega$ and $\Omega_{*}$ be two channels that coincide from some cross-section onwards. More precisely, let $\Omega=\{F(\zeta) \mid \zeta \in S\}$, and assume that $\partial \Omega_{*}$ intersects every arc

$$
z=F(c+i \eta), \quad c=\text { const. } \geqslant a, \quad-1 \leqslant \eta \leqslant 1
$$

at its end points, and there only, the component $\Gamma_{*+}$ of $\partial \Omega_{*}$ intersecting the end point $\eta=1$. Let $F_{*}$ be such that $\Omega_{*}=\left\{F_{*}(w) \mid w \in S\right\}$, and define $v_{*}$ and $\psi$ on $\bar{S}_{a}$ by

$$
v_{*}(\zeta)=\operatorname{Im}\left(F_{*}^{-1} \circ F^{\prime}\right)(\zeta)=\eta+\varphi(\zeta)
$$

Then, for each multi-index $\alpha$, there exists a constant $c_{\alpha}$ such that

$$
\left|\nabla^{\alpha} \psi(\zeta)\right| \leqslant c_{\alpha} \exp \left\{-\frac{\pi}{2}(\xi-a)\right\} \quad \text { on } \bar{S}_{a+1}
$$

Proof. For any $\phi \in C^{2}\left(\mathcal{S}_{a}\right) \cap C^{1}\left(\bar{S}_{a}\right)$, Green's identity implies that

$$
\phi\left(\zeta_{0}\right)=-\int_{S_{a}} K\left(\zeta_{0}, \zeta\right) \nabla^{2} \phi(\zeta)-\int_{\partial s_{a}} \frac{\partial K}{\partial n}\left(\zeta_{0}, \zeta\right) \phi(\zeta) d l
$$

where $n$ is the outward unit normal on $\partial S_{a}$, and $\partial / \partial n=n \cdot \nabla$. Now consider $\psi$. The hypothesis about $\partial \Omega_{*}$ ensures that $F\left(\bar{S}_{a}\right) \subset \bar{\Omega}_{*}$, so that $v_{*}$ and $\psi$ are well defined. Applying (A.16) to $\psi$, we note that $|\psi(\zeta)| \leqslant 2$ on $\bar{S}_{a}$, because $\left|\operatorname{Im} F_{*}^{-1}(z)\right| \leqslant 1$ on $\bar{\Omega}_{*}$ and $|\eta| \leqslant 1$ on $\bar{S}$; that $\nabla^{2} \psi=0$ in $S_{\alpha}$, because $v_{*}$ and $\eta$ are harmonic there; and that $\psi(\xi, \pm 1)=0, \xi \geqslant a$, because $\operatorname{Im} F_{*}^{-1}\left(\partial \Omega_{*}\right)= \pm 1$, with the upper sign corresponding to $\eta=1$. Hence

$$
\psi\left(\zeta_{0}\right)=\int_{-1}^{1} K_{\xi}\left(\zeta_{0}, a, \eta\right) \psi(a, \eta) d \eta
$$

with $|\psi(a, \eta)| \leqslant 2$. The result now follows from (A.15) (with $X=\pi a / 2$ ) and either (A.14b) or $(\mathrm{A} .14 \mathrm{c})$.

We shall need to discuss the potential function

$$
P\left(\zeta_{0}\right)=\int_{s_{a}} K\left(\zeta_{0}, \zeta\right) f(\zeta)
$$

of a density function $f$; for this, a natural setting is the space $C^{k+\mu}\left(\bar{S}_{a}\right)$ of functions $\phi: \bar{S}_{a} \rightarrow \mathbf{R}$ such that $\nabla^{\alpha} \phi, 0 \leqslant|\alpha| \leqslant k$, can be extended from $S_{a}$ to $\bar{S}_{a}$ as bounded continuous functions, derivatives of order $k$ being Hölder continuous on $\bar{S}_{a}$ with exponent $\mu \in(0,1)$. 
The norm is

where

$$
\|\phi\|_{k+\mu_{s}, S_{a}}=\sup _{\zeta \in S_{\alpha,|\alpha| \leqslant k}}\left|\nabla^{\alpha} \phi(\zeta)\right|+\max _{|\alpha|=k}\left[\nabla^{\alpha} \phi\right]_{\mu, S_{a}}
$$

$$
[\psi]_{\mu, S_{a}}=\sup _{\zeta, \zeta_{0} \in S_{a}} \frac{\left|\psi(\zeta)-\psi\left(\zeta_{0}\right)\right|}{\left|\zeta-\zeta_{0}\right|^{\mu}}, \quad 0<\left|\zeta-\zeta_{0}\right| \leqslant 1, \quad 0<\mu<1 .
$$

The following result is of a classical type (cf. [19], [22]; also [3]); some steps of the proof are given in Appendix B.

Lемм A.2. Let $S_{a}$ be a half-strip with $a \geqslant 1$; let $\varrho$ be the weight function defined by $\varrho(\zeta)=\xi^{c}, c \geqslant 0 ;$ and let $P$ be the potential of a density $f$, as in (A.17). If $\varrho f \in C^{0+\mu}\left(\bar{S}_{a}\right)$, then $\varrho P \in C^{2+\mu}\left(\bar{S}_{a+1}\right)$, and there exists a constant $k$ (depending only on $c$ and $\mu$ ) such that

$$
\|\varrho P\|_{2+\mu, s_{a+1}} \leqslant k\left\|_{\varrho}\right\|_{0+\mu_{s} s_{a}} \text {. }
$$

We cannot claim that $P \in C^{2+\mu}\left(\bar{S}_{a}\right)$, because in general second derivatives of $P$ have a logarithmic singularity at the corners $(a, \pm 1)$ of $\bar{S}_{a}$.

THEOREM A.3. Let $\mathcal{D}$ be an open set in the $\zeta$-plane that contains the closure $\bar{S}_{a}, a \geqslant 1$, of a half-strip. Let $E$ be a conformal, one-to-one function on $\mathcal{D}$, with range $R=E(\mathcal{D})$ in the $z$-plane, that is related in the following way to a channel $\Omega$. Each component, $\Gamma_{+}$or $\Gamma_{-}$, of $\partial \Omega$ intersects every arc $E(\{\xi=$ const. $\geqslant a\})$ in $R$ exactly once, in such a way that $E^{-1}\left(\Gamma_{+} \cap R\right)$ and $E^{-1}\left(\Gamma_{-} \cap \mathcal{R}\right)$ have respective representations, for $\xi \geqslant a$,

where

$$
\left.\begin{array}{r}
\eta=1+f_{+}(\xi) \quad \text { and } \eta=-1-f_{-}(\xi), \\
\left|f_{ \pm}^{(n)}(\xi)\right| \leqslant \text { const. } \xi^{-c}, \quad c>0, n=0,1,2,3 .
\end{array}\right\}
$$

Define $Q_{a}=\left\{\zeta \mid \xi>a,-1-f_{-}(\xi)<\eta<1+f_{+}(\xi)\right\}$, let $F$ be such that $\Omega=\{F(w) \mid w \in S\}$, and define $v_{*}$ and $\psi$ on $\bar{Q}_{a}$ by

$$
v_{*}(\zeta)=\operatorname{Im}\left(F^{-1} \circ E\right)(\zeta)=\eta+\psi(\zeta)
$$

Then $\varrho \psi \in C^{2+\mu}\left(\bar{Q}_{a+2}\right)$, where $\mu$ is any exponent in $(0,1)$, and $\varrho(\zeta)=\xi^{c} ;$ in particular,

$$
\left|\nabla^{\alpha} \psi(\zeta)\right| \leqslant \text { const. } \xi^{-c} \text { for } \zeta \in \bar{Q}_{a+2} \text { and }|\alpha| \leqslant 2
$$

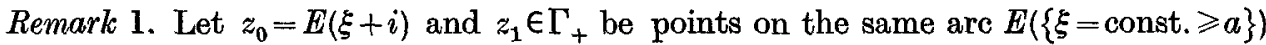
in $R$, and let $h_{E}=\left|E^{\prime}\right|$. In general, the hypothesis (A.18) does not require $\left|z_{0}-z_{1}\right|$ to be small, because this distance is approximately $h_{E}(\xi, 1)\left|f_{+}(\xi)\right|$, which may well tend to infinity as $\xi \rightarrow \infty$. 
Remark 2. In order that the hypothesis 'intersects ... exactly once' be satisfied, $\mathcal{D}$ will often be smaller than the largest set on which $E$ is conformal and one-to-one. For example, when $E$ is as in $(v)$, one can choose $\mathcal{D}$ to be the set $\xi>a-1,|\arg \zeta|<\pi / 2 m$; then $\mathcal{R}$ does not intersect $\partial\left(\Omega_{f} \backslash \Omega_{k}\right)$ for sufficiently large values of $a$.

Proof of Theorem A.3. (i) The function $y=\operatorname{Im} F^{-1}$ is the unique solution of the Dirichlet problem

$$
D^{2} v=0 \quad \text { in } \Omega,\left.\quad v\right|_{\Gamma_{+}}=1,\left.\quad v\right|_{\Gamma_{-}}=-1,
$$

subject to the condition $|v(z)| \leqslant 1$. We may, and shall, assume $a$ to be so large that $\left|f_{ \pm}(\xi)\right| \leqslant \frac{1}{2}$ for $\xi \geqslant a$; then the hypothesis about $\partial \Omega$ ensures that $E\left(\bar{Q}_{a}\right) \subset \bar{\Omega}$, so that $v_{*}$ and $\psi$ are well defined by (A.19), which implies that $|\psi(\zeta)| \leqslant 5 / 2$ on $\bar{Q}_{a}$ (since $\left|v_{*}(\zeta)\right| \leqslant 1$ and $|\eta| \leqslant 3 / 2$ there), and that

$$
\begin{gathered}
\nabla^{2} \psi=0 \text { in } Q_{a}, \\
\psi(\xi)=\left\{\begin{array}{l}
-f_{+}(\xi) \text { for } \xi \geqslant a, \quad \eta=1+f_{+}(\xi), \\
t_{-}(\xi) \text { for } \xi \geqslant a, \quad \eta=-1-f_{-}(\xi) .
\end{array}\right.
\end{gathered}
$$

Regularity theory for elliptic equations ([3], p. 668) then implies existence of a constant $k_{\mu}$ such that

$$
\|\psi\|_{2+\mu, Q_{a+1}} \leqslant k_{\mu}, \quad \mu \in(0,1)
$$

where we have used the bound $|\psi(\zeta)| \leqslant 5 / 2$ as well as (A.20).

(ii) Let $a+1=b$. We map $Q_{b}$ onto $S_{b}$ by the coordinate transformation

$$
r=\xi, \quad s=\left\{2 \eta-f_{+}(\xi)+f_{-}(\xi)\right\} /\left\{2+f_{+}(\xi)+f_{-}(\xi)\right\},
$$

(which is not conformall). Merely for ease of writing, we now suppose that $f_{+}=f_{-}=f$, and define

Then

$$
p(r)=1+f(r), \quad q(t)=-s p^{\prime}(r) / p(r)=-s f^{\prime}(r) / p(r), \quad t=(r, s) .
$$

and

$$
\frac{\partial}{\partial \xi}=\frac{\partial}{\partial r}+q(t) \frac{\partial}{\partial s}, \quad \frac{\partial}{\partial \eta}=\frac{1}{p(r)} \frac{\partial}{\partial s},
$$

where

$$
\nabla^{2}=\frac{\partial^{2}}{\partial \xi^{2}}+\frac{\partial^{2}}{\partial \eta^{2}}=\frac{\partial^{2}}{\partial r^{2}}+\frac{\partial^{2}}{\partial s^{2}}+L
$$

$$
L=2 q \frac{\partial^{2}}{\partial r \partial s}+\left(q^{2}+\frac{1}{p^{2}}-1\right) \frac{\partial^{2}}{\partial s^{2}}+\left(q_{r}+q q_{s}\right) \frac{\partial}{\partial s}
$$

If $c_{L}$ denotes any coefficient in $L$, then it follows from (A.18) that $\varrho c_{L} \in C^{1}\left(\bar{S}_{b}\right)$, where $\varrho(t)=r^{c}$; and (A.21) now implies that $\varrho L \phi \in C^{0+\mu}\left(\bar{S}_{b}\right)$, where $\phi(t)=\psi(\zeta)$. By $(\mathrm{A} .20)$, 


$$
\begin{gathered}
\phi_{r r}+\phi_{s s}=-L \phi \text { in } S_{b}, \\
\phi(t)=\left\{\begin{array}{c}
\mp f(r) \text { for } r \geqslant b, s= \pm 1 \text { respectively } \\
g(s), \text { say, } \text { for } r=b,-1 \leqslant s \leqslant 1,
\end{array}\right.
\end{gathered}
$$

where $|\phi(t)| \leqslant 5 / 2$ on $\bar{S}_{b}$. Then (A.16) shows that $\phi$ has the representation, on $\bar{S}_{b}$,

where

$$
\phi(t)=\sum_{j=1}^{3} \phi_{j}(t)
$$

$$
\begin{aligned}
& \phi_{1}\left(t_{0}\right)=\int_{s_{b}} K\left(t_{0}, t\right) L \phi(t), \\
& \phi_{2}\left(t_{0}\right)=-f\left(r_{0}\right) s_{0}-\int_{S_{b}} K\left(t_{0}, t\right) f^{\prime \prime}(r) s, \\
& \phi_{3}\left(t_{0}\right)=\int_{-1}^{1} K_{r}\left(t_{0}, b, s\right)\{g(s)+f(b) s\} d s .
\end{aligned}
$$

Here $\phi_{3}\left(t_{0}\right)$ has the form considered in Theorem A.1, and is exponentially small as $r_{0} \rightarrow \infty$. Application of Lemma A.2 to $\phi_{1}$ and $\phi_{2}$ shows that $\varrho \phi \in C^{2+\mu}\left(\bar{S}_{b+1}\right)$; reversing the coordinate transformation, we obtain the result of the theorem.

Q.E.D.

Remark 3. Suppose that the conditions in (A.18) can be refined to

$$
\left|f_{ \pm}^{(n)}(\xi)\right| \leqslant \text { const } \xi^{-c_{n}}, \quad n=0,1,2,3, \quad \text { where } \quad c_{n+1} \geqslant c_{n}>0, \quad n=0,1,2 .
$$

This is the case in (v), where $c_{n}=n+2$. Then the result can also be refined; for example, $\nabla^{\alpha} \psi$ is $O\left(\xi^{-2 c_{0}}+\xi^{-c_{1}}\right)$ for $|\alpha|=2$. To prove this, one uses the theorem as stated and with $c=c_{0}$, to estimate once more (and more carefully) $L \phi$ and other terms in the expressions for the $\phi_{j}$. In principle, this refinement is useful for applications, but, for simple cases like the channels $\Omega_{f}$, it is not needed.

Remark 4. Let $E$ and $Q_{a}$ be as in Theorem A.3. We shall say that the end map $E$ is of type $T$ on $Q_{a}$ (where $T=\mathrm{I}, \mathrm{II}$, III or $\mathrm{I}^{\prime}$ ) if $h_{E}=\left|E^{\prime}\right|$ enjoys on $Q_{a}$ the properties demanded of $h$ on $S$ in order that $\Omega$ be of type $T$.

CoROLLARY A.4. Under the hypotheses of Theorem A.3, let $E$ be of type $T$ on $Q_{a}$, and let $\varrho(\zeta)=\xi^{c}$. Then, as regards its downstream end, the channel $\Omega$ is of type $T$, provided that

(a) $1 / \varrho h_{E} \in L_{2}\left(Q_{a}\right)$ when $T=\mathrm{II}$ (for this, it suffices that $c>\frac{1}{2}$ );

(b) $c>\frac{1}{2}$ when $T=$ III. 
Proof. Let $h(w)=\left|F^{\prime}(w)\right|=|d z / d w|$, and define $h_{*}$ on $\bar{Q}_{a}$ by

$$
h_{*}(\zeta)=\left(h \circ F^{-1} \circ E\right)(\zeta)=\frac{|d z| d \zeta \mid}{|d w| d \zeta \mid}=\frac{h_{E}(\zeta)}{|d w| d \zeta \mid}
$$

where

$$
|d w / d \zeta|^{2}=\psi_{\xi}^{2}+\left(1+\psi_{\eta}\right)^{2}
$$

by (A.19). In place of the $|\nabla h|$ in Definitions 1.1 and 5.1, we now have

$$
\left|\left(h_{u}, h_{v}\right)\right|=\frac{\left|\left(h_{* \xi}, h_{* \eta}\right)\right|}{|d w / d \zeta|}=\frac{1}{|d w / d \zeta|^{2}}\left|\left(h_{E \xi}, h_{E \eta}\right)-\frac{h_{E} \Psi}{|d w / d \zeta|^{2}}\right|
$$

where

$$
\Psi=\left(\psi_{\xi} \psi_{\xi \xi}+\left(1+\psi_{\eta}\right) \psi_{\xi \eta}, \psi_{\xi} \psi_{\xi \eta}+\left(1+\psi_{\eta}\right) \psi_{\eta \eta}\right)
$$

Let $R_{a}=F^{-1} \circ E\left(Q_{a}\right)$; this is a subset of the strip $S$ in the $w$-plane that contains some half-strip $S_{d}$, and

$$
\int_{R_{a}}() d u d v=\int_{Q_{a}}()\left|\frac{d w}{d \zeta}\right|^{2} d \xi d \eta .
$$

It is straightforward to check, by means of these formulae, that $h$ has the desired properties, given our hypotheses on $h_{E}$ and $c$, and the result of Theorem A.3 for $\psi$. The assumptions (a) and (b) are made because of the $\Psi$-term above, to ensure that $|\Psi| / h \in L_{2}$, in order that $\left|\nabla_{w}(1 / h)\right| \in L_{2}$, when $T=\mathrm{II}$; and to ensure that $|\Psi| \in L_{2}$, in order that $\left|\nabla_{w} \log h\right| \in L_{2}$, when $T=$ III.

Q.E.D.

Corollary A.5. The channels $\Omega_{f}$ defined by (1.6) are of type $I^{\prime}$ if $k_{j}>\frac{1}{3}$, of type $I I$ if $k_{j}<1$, and of type $I I I$ if $k_{j} \leqslant \frac{1}{3}(j=1,2$ throughout $)$.

Proof. We use the results of (v) to (vii) in Corollary A.4. For $k_{j}=1$, Theorem A.1 can be used.

Q.E.D.

\section{Appendix B. Some details omitted from proofs}

(i) Remainder terms in the proof of Lemma 3.3. We recall that $R_{1}$ comes from $(2.20)$, $R_{2}$ from integration by parts of the integral in (3.9), and $-R_{3}$ from the same integral when 1 and $\psi / h$ replace $1 / h^{2}$ and $\psi$, respectively. We find that

$$
\begin{aligned}
& R_{1}=-\int_{S} \frac{\varrho}{h^{2}} Q_{\eta} \varkappa\left(\psi_{\xi}^{2}+\psi_{\eta}^{2}\right)+\int_{S} \frac{\varrho^{\prime}}{h^{2}} Q\left\{\psi_{\eta} \psi_{\xi \xi}-\psi_{\xi} \psi_{\xi \eta}+\lambda\left(\psi_{\xi}^{2}+\psi_{\eta}^{2}\right)\right\} \\
& R_{2}=\int_{S}\left\{-\frac{\varrho^{\prime}-2 \varrho \varkappa}{h^{2}} Q_{\eta} \psi_{\eta}^{2}+\frac{2 \varrho \lambda}{h^{2}} Q_{\eta} \psi_{\xi} \psi_{\eta}\right\} \\
& R_{3}=\int_{S} \frac{\varrho^{\prime}}{h^{2}} Q_{\eta}\left(\psi_{\eta}-\lambda \psi\right)^{2}+\int_{S} \frac{\varrho}{h^{2}} Q_{\eta \eta}\left(\varkappa \psi \psi_{\eta}+\lambda \psi \psi_{\xi}-\varkappa \lambda \psi^{2}\right)
\end{aligned}
$$


(ii) The triple product $\{w, u, u\}$ in terms of stream functions. Let $w=\left(\chi_{y},-\chi_{x}\right)$ and $u=\left(\Psi_{y},-\Psi_{x}\right)$, where $\chi \in C_{0}^{\infty}(\Omega)$ because $w \in J(\Omega)$ in $(3.1 \mathrm{a})$. Starting from the definition in section 2.1 , we obtain

$$
\begin{aligned}
\{w, u, u\} & =\int_{\Omega}\left\{\chi_{y}\left(\Psi_{y} \frac{\partial}{\partial x}-\Psi_{x} \frac{\partial}{\partial y}\right) \Psi_{y}+\chi_{x}\left(\Psi_{y} \frac{\partial}{\partial x}-\Psi_{x} \frac{\partial}{\partial y}\right) \Psi_{x}\right\} \\
& =\int_{\Omega}\left\{\chi_{y}\left[\left(\frac{1}{2} \Psi_{y}^{2}\right)_{x}-\Psi_{x} \Psi_{y y}\right]+\chi_{x}\left[\Psi_{y} \Psi_{x x}-\left(\frac{1}{2} \Psi_{x}^{2}\right)_{y}\right]\right\} \\
& =\int_{\Omega}\left\{\chi_{x}\left(\frac{1}{2} \Psi_{y}^{2}\right)_{y}-\chi_{y} \Psi_{x} \Psi_{y y}+\chi_{x} \Psi_{y} \Psi_{x x}-\chi_{y}\left(\frac{1}{2} \Psi_{x}^{2}\right)_{x}\right\} \\
& =\int_{\Omega}\left(\chi_{x} \Psi_{y}-\chi_{y} \Psi_{x}\right) D^{2} \Psi^{2}
\end{aligned}
$$

Now transforming to $S$, one can use the fact that $\chi_{x} \Psi_{y}-\chi_{y} \Psi_{x}$ is a Jacobian.

(iii) Steps in the proof of Lemma A.2. Let $\left|\dot{\zeta}-\zeta_{0}\right|=r$. From the formulae (A.14a) and (A.15), we deduce that the Green function $K$ is no worse on $\bar{S}_{a} \times \bar{S}_{a}$ than the fundamental singularity $-(1 / 2 \pi) \log r$. Indeed, let $\zeta_{1}$ and $\zeta_{2}$ denote the reflections of $\zeta_{0}$ in the lines $\eta= \pm 1$, let $\zeta_{j+3}(j=0,1,2)$ be the reflection of $\zeta_{j}$ in the line $\xi=a$, and let $r_{j}=\left|\zeta-\zeta_{j}\right| \geqslant r(j=1, \ldots, 5)$; then $2 \pi K\left(\zeta, \zeta_{0}\right)-\log \left(r_{1} r_{2} r_{3} / r_{4} r_{5} r\right)$ is real analytic on $\bar{S}_{a} \times \bar{S}_{a}$. Also, (A.14b, c) show exponential decay for large values of $\left|\xi-\xi_{0}\right|$. Hence there exist constants $c, c_{\alpha \beta}$ and $k_{\alpha \beta}$ such that

$$
\left|\nabla_{0}^{\alpha} \nabla^{\beta} K\left(\zeta_{0}, \zeta\right)\right| \leqslant \begin{cases}c(1+|\log r|), & |\alpha+\beta|=0, \quad 0<r \leqslant 3 \\ c_{\alpha \beta} r^{-|\alpha+\beta|},|\alpha+\beta|>0, & 0<r \leqslant 3, \\ k_{\alpha \beta} \exp \left(-\frac{\pi}{2}\left|\xi-\xi_{0}\right|\right), & \left|\xi-\xi_{0}\right| \geqslant \frac{1}{2} .\end{cases}
$$

We estimate all integrals but one by taking the modulus of the integrand, and by representing $S_{a}$ as the union of the (overlapping) subsets characterized by $r \leqslant 5^{\frac{1}{3}}$ and by $\left|\xi-\xi_{0}\right| \geqslant 1$. (Subsets slightly larger than this are considered in (B.1), in order that $K^{(2)}\left(\zeta_{1}, \zeta\right)$ can be estimated in (B.2) when we partition $S_{a}$ by reference to $\zeta_{0}$.)

Now define

$$
\begin{aligned}
\Phi\left(\zeta_{0}\right) & =\int_{S_{a}} K\left(\zeta_{0}, \zeta\right) \\
& =\frac{1}{2}\left(1-\eta_{0}^{2}\right)-\frac{16}{\pi^{3}} \sum_{n=1,3,5, \ldots} \frac{1}{n^{3}} \exp \left\{-\frac{n \pi}{2}\left(\xi_{0}-a\right)\right\} \sin \left\{\frac{n \pi}{2}\left(\eta_{0}+1\right)\right\},
\end{aligned}
$$

and let $(.)^{(n)}$ denote any partial derivative of order $n$ with respect to $\zeta_{0}$ (or, later, with respect to $\left.\zeta_{1}\right)$. The following differentiation formulae are justified, when $f \in C^{0+\mu}\left(\overline{S_{a}}\right)$, as the 
limits of finite differences:

$$
\begin{aligned}
& P^{(1)}\left(\zeta_{0}\right)=\int_{S_{a}} K^{(1)}\left(\zeta_{0}, \zeta\right) f(\zeta) \\
& P^{(2)}\left(\zeta_{0}\right)=\int_{S_{a}} K^{(2)}\left(\zeta_{0}, \zeta\right)\left\{f(\zeta)-f\left(\zeta_{0}\right)\right\}+\Phi^{(2)}\left(\zeta_{0}\right) f\left(\zeta_{0}\right), \quad \xi_{0}>a
\end{aligned}
$$

The most elaborate part of our task is the proof of Hölder continuity, with exponent $\mu$, of second derivatives. For this we consider two points $\zeta_{1}$ and $\zeta_{0}$ such that $\left|\zeta_{1}-\zeta_{0}\right|=\delta$, $0<\delta \leqslant \frac{1}{2}$, and use the formula

$$
\begin{aligned}
P^{(2)}\left(\zeta_{1}\right)-P^{(2)}\left(\zeta_{0}\right)=\int_{r<2 \delta} & K^{(2)}\left(\zeta_{1}, \zeta\right)\left\{f(\zeta)-f\left(\zeta_{1}\right)\right\}-\int_{r<2 \delta} K^{(2)}\left(\zeta_{0}, \zeta\right)\left\{f(\zeta)-f\left(\zeta_{0}\right)\right\} \\
& +\int_{r>2 \delta}\left\{K^{(2)}\left(\zeta_{1}, \zeta\right)-K^{(2)}\left(\zeta_{0}, \zeta\right)\right\}\left\{f(\zeta)-f\left(\zeta_{1}\right)\right\} \\
& -\left\{f\left(\zeta_{1}\right)-f\left(\zeta_{0}\right)\right\} \int_{r>2 \delta} K^{(2)}\left(\zeta_{0}, \zeta\right) \\
& +\Phi^{(2)}\left(\zeta_{1}\right)\left\{f\left(\zeta_{1}\right)-f\left(\zeta_{0}\right)\right\}+\left\{\Phi^{(2)}\left(\zeta_{1}\right)-\Phi^{(2)}\left(\zeta_{0}\right)\right\} f\left(\zeta_{0}\right) .
\end{aligned}
$$

The two integrals over $r<2 \delta$ can be bounded separately; each is not greater than a constant times $\xi_{0}^{-c} \delta^{\mu}$, under the hypothesis of Lemma A.2. The third integral is shown, by the method mentioned above, to be of the same order. It is the last integral, of $K^{(2)}\left(\zeta_{0}, \zeta\right)$ alone, that requires care if the exponent $\mu$ is to be recovered for second derivatives. Now $\xi$ and $\xi_{0}$ appear in (A.15) only in the combinations $\xi \mp \xi_{0}$, and $G_{\eta_{0} \eta_{0}}=-G_{\xi_{0} \xi_{0}}$ for $\zeta \neq \zeta_{0}$; therefore, we can always write $K^{(2)}$ as a derivative with respect to $\xi$ of some $\hat{K}^{(1)}$, where $\hat{K}$ is a linear combination of the two functions $\tilde{G}$ in (A.15). Hence the integral can be reduced to one of $\hat{K}^{(1)}$ over the line segment $\{a\} \times(-1,1)$ and over that part of the circle $r=2 \delta$ within $S_{a}$; in this form, it is readily bounded by a constant.

\section{References}

[1] Adams, R. A., Sobolev spaces. Academic Press, 1975.

[2] Agmon, S., The $L_{p}$ approach to the Dirichlet problem. Ann. Scuola Norm. Sup. Pisa, (3) 13 (1959), 405-448.

[3] Agmon, S., Douglis, A. \& Nirenberg, L., Estimates near the boundary for solutions of elliptic partial differential equations satisfying general boundary conditions. I. Comm. Pure Appl. Math., 12 (1959), 623-727.

[4] Amrck, C. J., Steady solutions of the Navier-Stokes equations in unbounded channels and pipes. Ann. Scuola Norm. Sup. Pisa, (4) 4 (1977), 473-513.

[5] - Properties of steady Navier-Stokes solutions for certain unbounded channels and pipes. Nonlinear Analysis, Theory, Methods and Applications, 2 (1978), 689-720. 
[6] BABENko, K. I., On stationary solutions of the problem of flow past a body by a viscous incompressible fluid. Mat. Sb., 91 (1973), 3-26.

[7] Carathéodory, C., Conformal representation. Cambridge, 1932.

[8] Cattabriga, L., Su un problema al contorno relativo al sistema di equazioni di Stokes. Rend. Sem. Mat. Padova, 31 (1961), 1-33.

[9] FIns, R., Stationary solutions of the Navier-Stokes equations. Amer. Math. Soc., Proc. Symp. Appl. Math., 17 (1965), 121-153.

[10] - Mathematical questions relating to viscous fluid flow in an exterior domain. Rocky Mountain J. Math., 3 (1973), 107-140.

[11] FinN, R. \& SMITH., D. R., On the stationary solutions of the Navier-Stokes equations in two dimensions. Arch. Rat. Mech. Anal., 25 (1967), 26-39.

[12] Fraenkel, L. E., On a theory of laminar flow in channels of a certain class. Proc. Camb. Phil. Soc., 73 (1973), 361-390.

[13] - Boundary-value problems for the steady Navier-Stokes equations. Zeitschr. Angew. Math. Mech., 56 (1976), T7-T16.

[14] Fraenkel, L. E. \& Eagles, P. M., On a theory of laminar flow in channels of a certain class. II. Math. Proc. Camb. Phil. Soc., 77 (1975), 199-224.

[15] Friedman, A, On the regularity of the solutions of nonlinear elliptic and parabolic systems of partial differential equations. J. Math. Mech., 7 (1958), 43-59.

[16] Fujita, H., On the existence and regularity of the steady-state solutions of the NavierStokes equations. J. Fac. Sci. Univ. Tokyo Sect. I, 9 (1961), 59-102.

[17] Grlbara, D. \& Weinberaer, H. F., Asymptotic properties of Leray's solution of the stationary two-dimensional Navier-Stokes equations. Russ. Math. Surv., 29, No. 2 (1974), 109-123.

[18] - Asymptotic properties of steady plane solutions of the Navier-Stokes equations with bounded Dirichlet integral. Ann. Scuola Norm. Sup. Pisa, (4) 5 (1978), 381-404.

[19] Günter, N. M., Potential theory. Ungar, 1967.

[20] Hardy, G. H., Litruewoon, J. E. \& Pólya, G., Inequalities. Cambridge, 1952.

[21] Heywood, J. G., On uniqueness questions in the thecry of viscous flow. Acta Math., I36 (1976), 61-102.

[22] KellogG, O. D., Foundations of potential theory. Springer, 1929.

[23] Ladyzhenskaya, O. A., The mathematical theory of viscous incompressible flow. Second Edition, Gordon and Breach, 1969.

[24] NEČas, J., Les méthodes directes en théorie des équations elliptiques. Masson, 1967.

[25] Protier, M. H. \& Weinberger, H. F., Maximum principles in differential equations. Prentice-Hall, 1967.

[26] Temam, R., Navier-Stokes equations: theory and numerical analysis. North-Holland, 1977.

[27] Titchmansh, E. C., The theory of functions. Oxford, 1932.

\section{References added in proof}

[28] Ladyzhmskaya, O. A. \& Solonnikov, V. A., On the solvability of boundary-and initialboundary-value problems for the Navier-Stokes equation in domains with noncompact boundary. Vestnik Leningrad Univ. Mat. Meh. Astron., 13 (1977), 39-47.

[29] — On some problems of vector analysis and generalized formulations of boundaryvalue problems for the Navier-Stokes equation. Zap. Naučn. Sem. Leningrad Otdel. Mat. Inst. Steklov (LOMI), 59 (1976), 81-116.

[30] Maslennizova, V. N. \& Bogovsku, M. E., On the density of solenoidal vector fields with compact support. Sibirsk. Mat. Z., 19 (1978), 1092-1108. 
The outline of previous existence theory, in section 1.1, should certainly include reference to $[28]$.

[28] concerns certain domains in $\mathbf{R}^{2}$ or $\mathbf{R}^{3}$ with any (finite) number of 'exits' or ducts extending to infinity (a channel has two). The existence of generalized steady solutions is proved for arbitrary Reynolds numbers (that is, for arbitrary compatible flux constants and viscosity). Our flux carrier in Lemma 3.2 has something in common with those constructed earlier by Ladyzhenskaya and Solonnikov in their more general setting, but in their paper the 'exits' or ducts in $\mathbf{R}^{2}$ must widen, at large distances, more strongly than is necessary for our channels of type $I$ or type $I^{\prime}$, and pointwise decay of the velocity is not considered.

[29] and [30] are primarily concerned with Heywood's question in [21]: for what domains $\Omega$ does the completion of $C_{0}^{\infty,}$, sol $\left(\Omega \rightarrow \mathbf{R}^{N}\right)$ contain all solenoidal vector fields in the completion of $C_{0}^{\infty}\left(\Omega \rightarrow \mathbf{R}^{N}\right)$ ? (Here completion is either in the Dirichlet norm for both sets, or else in the full norm of $W_{2}^{1}\left(\Omega \rightarrow \mathbf{R}^{N}\right)$ for both sets. For channels, ducts and the like, vector fields in the former space carry no flux, while those in the latter space may carry flux for certain domains.) The two papers cited add significantly to Heywood's own answers to this question. As it happens, Maslennikova and Bogovskii introduce channels and tubes of 'types I to III', but their definitions are entirely different from those in the present paper; they prove (among much else) that equality of the two function spaces, when $\Omega$ is a symmetrical channel or tube, implies certain integral properties of the cross-sectional area.

Received December 4, 1978 